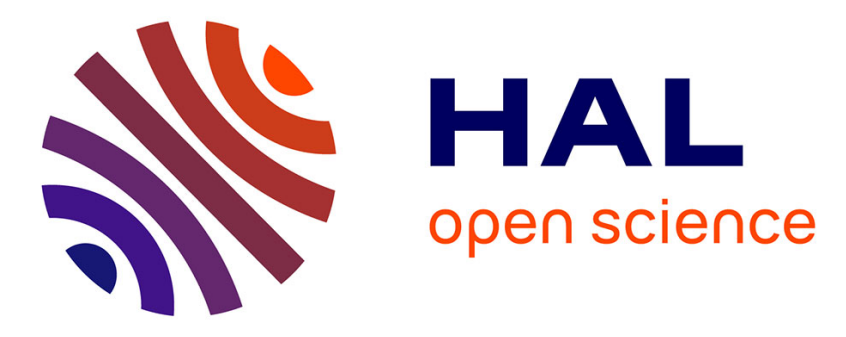

\title{
Drying colloidal systems: Laboratory models for a wide range of applications
}

Patrice Bacchin, David Brutin, Anne Davaille, Erika Di Giuseppe, Xiao Dong

Chen, Ioannis Gergianakis, Frédérique Giorgiutti-Dauphiné, Lucas Goehring, Yannick Hallez, Rodolphe Heyd, et al.

\section{To cite this version:}

Patrice Bacchin, David Brutin, Anne Davaille, Erika Di Giuseppe, Xiao Dong Chen, et al.. Drying colloidal systems: Laboratory models for a wide range of applications. European Physical Journal E: Soft matter and biological physics, 2018, 41 (8), pp.94. 10.1140/epje/i2018-11712-x . hal-01864878

\section{HAL Id: hal-01864878 https://hal.science/hal-01864878}

Submitted on 30 Aug 2018

HAL is a multi-disciplinary open access archive for the deposit and dissemination of scientific research documents, whether they are published or not. The documents may come from teaching and research institutions in France or abroad, or from public or private research centers.
L'archive ouverte pluridisciplinaire HAL, est destinée au dépôt et à la diffusion de documents scientifiques de niveau recherche, publiés ou non, émanant des établissements d'enseignement et de recherche français ou étrangers, des laboratoires publics ou privés.

\section{(ㅇ)(1) $\$$}

Distributed under a Creative Commons Attribution - NonCommercial - NoDerivatives| 4.0 


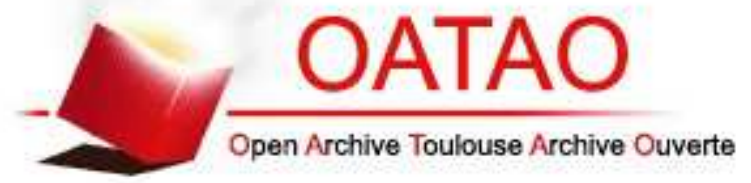

Open Archive Toulouse Archive Ouverte

OATAO is an open access repository that collects the work of Toulouse researchers and makes it freely available over the web where possible

This is an author's version published in: http://oatao.univ-toulouse.fr/ $\mathrm{n}^{\circ}$ de post : 20760

\section{Official URL:}

http://dx.doi.org/10.1140/epje/i2018-11712-x

\section{To cite this version:}

Bacchin, Patrice and Brutin, David and Davaille, Anne and Di Giuseppe, Erika and Dong Chen, Xiao and Gergianakis, loannis and Giorgiutti-Dauphiné, Frédérique and Goehring, Lucas and Hallez, Yannick and Heyd, Rodolphe and Jeantet, Romain and Le floc-Fouéré, Cécile and Meireles-Masbernat, Martine and Mittelstaedt, Eric and Nicloux, Céline and Ludovic, Pauchard and Saboungi, Marie-Louise Drying colloidal systems: Laboratory models for a wide range of applications. (2018) European Physical Journal E, 41 (94). 1-34. ISSN $1292-8941$

Any correspondence concerning this service should be sent to the repository administrator: tech-oatao@listes-diff.inp-toulouse.fr 


\title{
Drying colloidal systems: Laboratory models for a wide range of applications
}

Patrice Bacchin ${ }^{1}$, David Brutin ${ }^{2}$, Anne Davaille ${ }^{3}$, Erika Di Giuseppe ${ }^{4}$, Xiao Dong Chen $^{5}$, Ioannis Gergianakis, Frédérique Giorgiutti-Dauphiné ${ }^{3}$, Lucas Goehring ${ }^{6}$, Yannick Hallez ${ }^{1}$, Rodolphe Heyd ${ }^{7}$, Romain Jeantet ${ }^{8}$, Cécile Le Floch-Fouéré ${ }^{8}$, Martine Meireles ${ }^{1}$, Eric Mittelstaedt ${ }^{9}$, Céline Nicloux ${ }^{10}$, Ludovic Pauchard ${ }^{3, a}$, and Marie-Louise Saboungi ${ }^{11}$

1 Laboratoire de Génie Chimique, Université de Toulouse, CNRS, INPT, UPS, Toulouse, France

2 Aix-Marseille University, IUSTI UMR CNRS 7343, Marseille, France

3 Laboratoire FAST, UMR 7608 CNRS - Univ. Paris-Sud, Université Paris-Saclay, 91405, Orsay, France

4 MINES ParisTech, PLS Research University, CEMEF - Centre de mise en forme des matériaux, UMR CNRS 7635, CS 10207, 06904 Sophia Antipolis Cedex, France

5 Suzhou Key Lab of Green Chemical Engineering, School of Chemical and Environmental Engineering, College of Chemistry, Chemical Engineering and Material Science, Soochow University, Suzhou, China

${ }^{6}$ School of Science and Technology, Nottingham Trent University, Clifton Lane, Nottingham NG11 8NS, UK

7 LAMPA, Arts et Métiers ParisTech, 2, Boulevard du Ronceray, BP 93525, cedex 01, F-49035 Angers, France

8 Agrocampus Ouest, INRA, UMR STLO, Rennes, France

9 Department of Geological Sciences, University of Idaho, Moscow, ID, USA

10 Institut de Recherche Criminelle de la Gendarmerie Nationale, 5, Boulevard de l'Hautil, Pontoise, France

11 Institut de Minéralogie de Physique des Matériaux et de Cosmochimie (IMPMC); CNRS UMR7590 - Université Pierre et Marie Curie, Case 115, 4, place Jussieu, 75005 Paris, France

\begin{abstract}
The drying of complex fluids provides a powerful insight into phenomena that take place on time and length scales not normally accessible. An important feature of complex fluids, colloidal dispersions and polymer solutions is their high sensitivity to weak external actions. Thus, the drying of complex fluids involves a large number of physical and chemical processes. The scope of this review is the capacity to tune such systems to reproduce and explore specific properties in a physics laboratory. A wide variety of systems are presented, ranging from functional coatings, food science, cosmetology, medical diagnostics and forensics to geophysics and art.
\end{abstract}

\section{Introduction}

The drying of complex fluids, colloidal dispersions and polymer solutions, involves a large number of physical and chemical processes. The complex spatial and temporal evolutions of these systems during this process depend in particular on solvent diffusion, mass transfer at the vapour-medium interface and phase transitions. Drying is frequently observed over a wide variety of scientifically and technologically important systems: it is involved, for example, in the entire process of coating formation and significantly affects the quality of the final films. Furthermore, due to the number and nature of the components involved, the drying of complex fluids offers a way to high-

\footnotetext{
a e-mail: ludovic.pauchard@u-psud.fr
}

light and analyze processes that take place on time and length scales not normally accessible. The capacity to reproduce specific phenomena in such systems in a physics laboratory is the scope of this review. Several phenomena in a wide variety of systems at different time and length scales can be probed with analogous techniques as depicted in fig. 1.

The tunability of these systems emerges from the following properties. Polymer solutions and colloidal dispersions represent heterogeneous systems since from a microscopic point of view the components are organized into well-defined structures at the supramolecular scale. An important feature of the resulting structures is high sensitivity to weak external actions caused by mechanical stresses as well as by chemical processes. Among complex fluids, colloids are stable dispersions of minute particles 


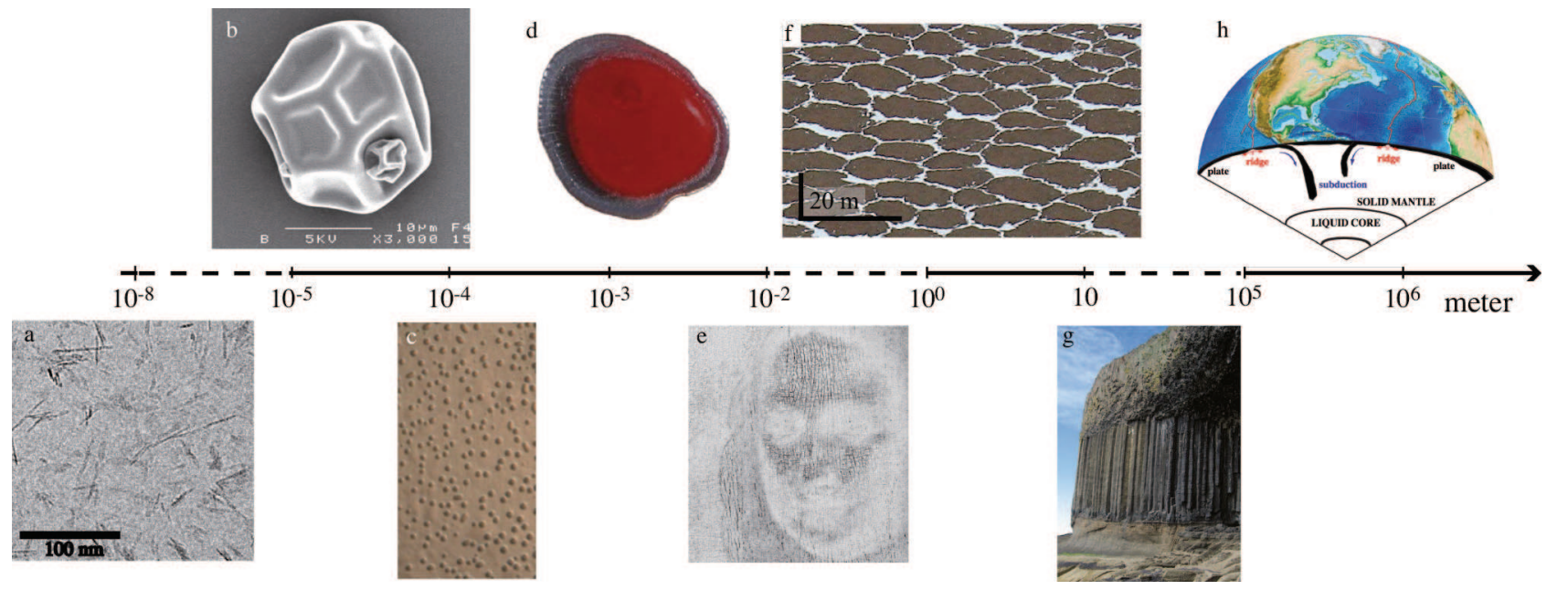

Fig. 1. (a) TEM image of a dried boehmite nanorod suspension [1]. (b) Casein micelles isolate. (c) Blisters in a cosmetic thin film. (d) Drying pool of blood. (e) Various crack patterns in Mona Lisa (reproduced from [2], with the permission of AIP Publishing). (f) Well-ordered permafrost in Beacon Valley, Antarctica. (g) Columns in lava near Fingal's Cave. (h) Convection in the solid mantle involving creation of a plate that will subduct back in the mantle.

in a continuous phase. A particular property of colloids, both organic and inorganic, is the extremely small particle size, typically ranging from $10 \mathrm{~nm}$ to $1 \mu \mathrm{m}$, exposing a large external surface per unit mass. Hence, the surface forces that dominate on colloidal lengthscales become a key factor in the stabilization or destabilization of a colloidal dispersion. In the absence of evaporation, if particles repel each other the dispersion remains stable, keeping the particles in suspension without sedimentation. Despite the complexity of the suspension due to the number and nature of its components, a colloidal dispersion can be described by effective potentials, most prominently for electrostatic repulsion and van der Waals attraction, the former serving to stabilize the dispersions and the latter being the primary cause for particle aggregation. The balance of these forces is addressed by the DLVO theory [3]. In addition, the stability of the colloidal dispersion can be strongly affected by altering the surface chemistry of the particles. For example, flocculation and aggregation of charged particles can be caused by increasing the ionic strength or lowering the surface charge. The control of the interparticle potential is one of the most important parameters for exploring different characteristics of the system and tuning its physical properties. Among the possible bonds between particles are depletion interactions, hydrogen bonds and hydrophobic effects at high concentrations. In polymer-colloid mixtures steric repulsion due to the presence of polymer chains or ligands serves to stabilize neighbouring particles. In the case of solvent flow, induced for example by evaporation, hydrodynamic forces between the dispersed particles can be entrained within the flow fields; liquid convection can be induced by many different causes including evaporation or temperature gradients, e.g. Marangoni flow, forced filtration or pressuredriven flow.

A large diversity of regimes are encountered during the drying process as a result of strong modifications of the chemical and physical properties of the systems. The

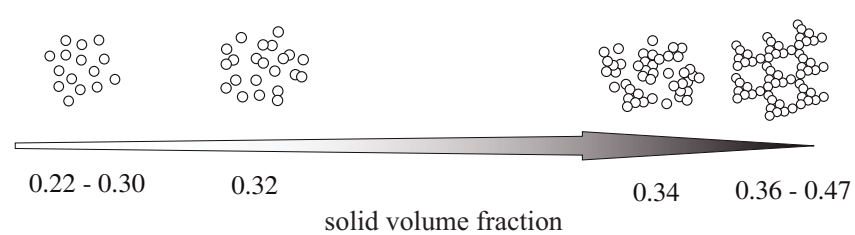

Fig. 2. Sketch of the increase of the particle concentration during the solvent removal of a colloidal dispersion. Aggregation or repulsive interaction result in different particles network depending on both the system and the evaporation rate. Adapted from Di Giuseppe et al. [4].

nanoscopic structure of the film evolves and crosses the liquid-solid boundary as the medium follows an out-ofequilibrium route governed mainly by thermodynamics and transport phenomena (fig. 2). At the macroscopic scale, the rheological properties of the medium, which change from a fluid phase into a viscoelastic, plastic, or brittle solid, result in hydrodynamic or mechanical instabilities.

Thus, the adaptability of the system in question opens up a multitude of possibilities of tuning it to exhibit specific properties. Based on laboratory experiments, an overview of the following problems will be presented:

- Fabrication of functional coatings exhibiting anticorrosion properties: in particular, when such homogeneous coatings are formed.

- Heterogeneities in cosmetic thin films by phase separation induced by evaporation: experimental modelling aims to highlight and understand the formation of such heterogeneities.

- Problems related to food science: the aim is to enhance the understanding of dairy colloids and processes involved in concentration ranges similar to those encountered in drying. 
- Problems related to medicine and/or forensics: experimental modelling aims to study dried blood that can be used for medical diagnostics, and/or determine the time and conditions in a homicide investigation.

- Problems related to art: experimental modelling aims to reproduce craquelures in paintings to determine the methods used by the artists, judge authenticity and determine their stability under environmental conditions.

- Experimental modelling provides a means to understand morphologies observed in nature such as columnar jointing in a fracture pattern.

- Problems related to surface phenomena at the geophysical scale: experimental modelling can provide quantitative access to phenomena in the earth or other planets over time and length scales inaccessible to humans.

\section{Functional coatings through colloidal dispersion evaporation}

Functional coatings are films deposited on surfaces to confer specific properties upon them, as chemical protection (resistance to oxidation), mechanical strength, optical properties (plasmonic effects for Raman spectroscopy), electrical properties, etc. Common examples of functional coatings are paints, anti-corrosion films for steel or ceramic membranes for fuel cells.

Such coatings are usually obtained by drying complex fluids or colloidal dispersions. The role of colloids is generally to provide their intrinsic properties to the film (optical response, conduction, ....) and/or to introduce an important specific surface area useful for heterogeneous reactions, catalysis, or mechanical strength. The success of the coating procedure depends on the homogeneity of the dry film obtained in the end. This homogeneity has been ensured for years by using Volatile Organic Compounds (VOC) as solvents because these liquids provide notably strong colloidal stability by enhancing electrostatic repulsions between nanoparticles and by solvating polymers efficiently. The new legislation for sustainable development urged industry to use environmentally friendly coatings only. This has motivated the shift from the $100 \%$ VOC dispersions towards zero-VOC waterborne coatings. The reduced stability associated to this change of solvent makes the fine control of the drying process all the more important to obtain an homogeneous film strongly attached to the substrate. Some examples of functional coatings with industrial relevance are briefly presented below.

The new legislation has motivated the shift from the $100 \%$ VOC paints used in the automobile industry towards waterborne coatings. These new systems are mainly suspensions of hard polymer particles, which yield hard top-coats upon drying. With such suspensions of hard nanoparticles, it is a challenge to produce flat, smooth, shiny, crack-free and durable films. So far industry still uses up to $15 \%$ VOC to soften the particles in order to achieve crack-free films. Though there is overall progress,

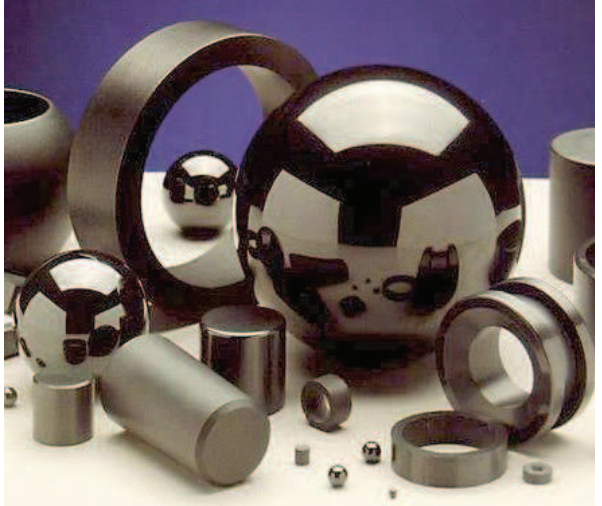

Fig. 3. Ball bearing parts reinforced by ceramic coatings $[5,6]$.

the remaining VOC are still harmful for the environment and particularly for the Ozone layer preservation. Thus avoiding cracks during drying of hard particles suspensions would actually help to preserve the environment!

Ceramic nanocomposites have been introduced in Solid Oxide Fuel Cells. The membranes carrying the oxygen ions are now made with yttria-doped zirconia. Currently these ceramics are made through processing and sintering of micrometer-sized dispersions of oxide particles. A major goal is the reduction of particle sizes down to about $100 \mathrm{~nm}$. This would allow sintering at lower temperatures and to produce thinner films, with higher conductance and better performance. However, the control of the deposition and drying stages for nanometric dispersions has proved to be difficult, due to premature aggregation of the particles. Similar problems occur in ceramic thermal barriers for aircraft engines.

Nano-ceramics functional coatings have also been designed to create tougher materials. Using nanoparticles is one route to improve the performance of ceramic materials that are submitted to high temperatures and intense wear as the ball bearing parts shown in fig. 3. Work along these lines has a large impact on the development of parts for car or aircraft engines. Here as well, it is expected that the use of particles in the $100 \mathrm{~nm}$ size range would make it possible to sinter at lower temperatures and to produce materials that are more ductile and less fragile than current ceramics. However, progress has been limited so far, because ceramics made from nanometric dispersions tend to crack upon drying. In the next section, we discuss in more details another type of functional coating used in aeronautics: anti-corrosion protections.

\subsection{Anti-corrosion coatings for aeronautics}

Specific anti-corrosion coatings are developed for the aeronautics industry as the integrity of the fuselage and wings metallic parts is affected by the external action of the environment. Coatings designed for corrosion protection must offer an effective physical barrier, impeding the access of aggressive species to the metallic interface, as well as a chemical one to provide a self-healing capacity in case of breaching. For many years, efficient anti-corrosion paints 


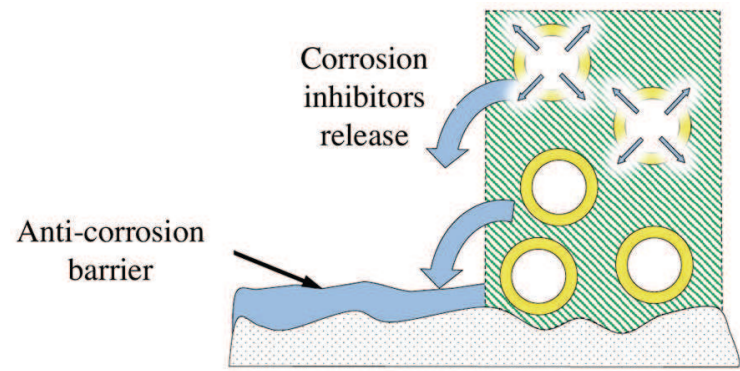

Fig. 4. Nano-container-based anti-corrosion coating: if the protecting functional film schematized on the right part is breached, the local change in ionic composition triggers a local desorption of corrosion inhibitors that will react with aggressive species to form a protective layer before the material is damaged.

were based on the use of chromium VI, a strong corrosion inhibitor. It has however been classified as carcinogenic, mutagenic or toxic for reproduction by the European regulation REACH and must be replaced. This last example is developed in more details in what follows.

A promising route to design new anti-corrosion coatings is to use cerium (Ce) as corrosion inhibitor. The large quantity of cerium salt to be used for an efficient protection is however not compatible with the mechanical stability of the coating. To overcome this limitation, cerium can be trapped on colloidal particles embedded in the coating. The latter are thus often referred to as "nano-reservoirs". cerium salt can be trapped between layers of particles, inside hollow capsules, or on the external surface of colloids. If the coating is breached, the physico-chemical environment near particles close to the breach changes, which triggers the local release of adsorbed cerium salt as described in fig. 4.

As an example, we report here some work on an anticorrosion coating based on the use of boehmite nanorods as cerium reservoirs. The protecting barrier is a film obtained through evaporation of a colloidal suspension containing both nanoparticles and ionic species. The colloidal suspension is initially stable due to repulsive particleparticle electrostatic interactions. At early times, physical drying concentrates particles, but also ionic species. Therefore, depending on initial physico-chemical conditions and hydrodynamics, the suspension can be destabilized before the film assembly is completed. In this case, particle aggregates can form in the fluid or a skin can appear at the free surface, leading to strongly heterogeneous or fragile coatings. In these unfavorable conditions, chemical drying also contributes to the assembly process, and in a unfavorable manner.

In the next two sections, we highlighted two important sources of film heterogeneity, namely the appearance of chemical drying and the flow-driven assembly.

\subsection{Thermodynamic view of the drying of a salty colloidal suspension}

Establishing the phase diagram of boehmite suspensions containing an added cerium salt (fig. 5) allows to estimate

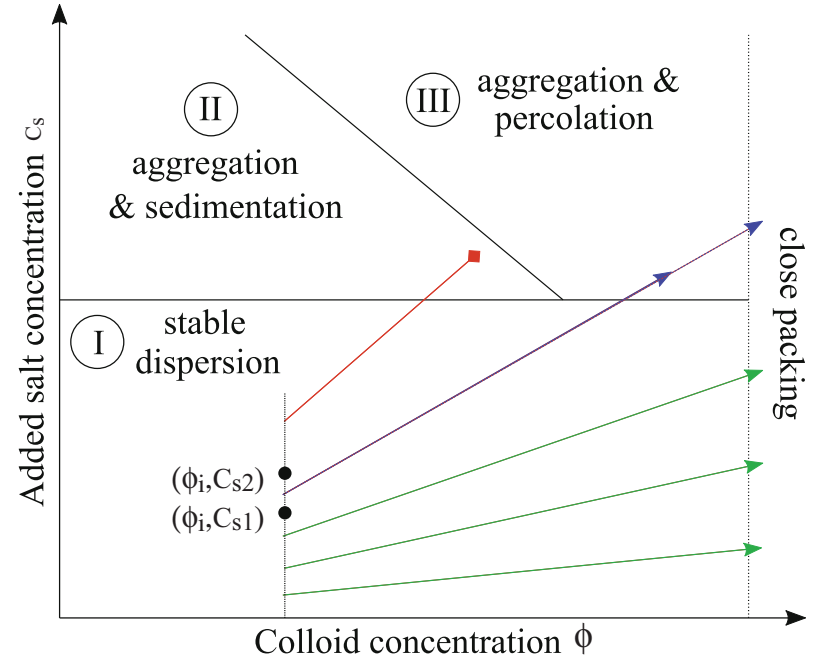

Fig. 5. Schematic phase diagram of a boehmite suspension with added cerium nitrate $\mathrm{Ce}\left(\mathrm{NO}_{3}\right)_{3}$ inspired from the experiments detailed in [1]. Colored lines are trajectories followed during drying for a unique initial colloid volume fraction $\phi_{i}$ and various initial salt concentrations $c_{s i}$.

if colloids can be brought to close contact before being destabilized in the bulk suspension. First focus on the diagram itself (black lines). For low added salt content (region I), colloids experience strong electrostatic repulsions that make the suspension stable and dispersed from dilute regimes up to close packing. Depending on the colloid volume fraction, the suspension can be liquid-like, glassy, or crystalline. Increasing the added salt content reduces the electrostatic repulsions, which leads to particle aggregation. At low colloid volume fraction isolated aggregates are formed and have time to sediment (region II). At high colloid volume fraction, the aggregation can take the form of percolation and a colloidal gel is formed (region III).

Consider drying of a suspension with colloid and salt concentrations $\phi(t)$ and $c_{s}(t)$, respectively. Because removal of solvent concentrates both colloids and salt at the same rate, we have $c_{s}(t)=\phi(t) c_{s i} / \phi_{i}$ where $\phi_{i}=\phi(t=0)$ and $c_{s i}=c_{s}(t=0)$. During drying, the trajectory of the suspension in the phase diagram is affine with slope $c_{s i} / \phi_{i}$. Considering an initial colloid volume fraction $\phi_{i}$, there are two critical initial salt concentrations denoted $c_{s 1}$ and $c_{s 2}$ in fig. 5. For $c_{s i}<c_{s 1}$ the trajectory remains in a dispersed phase until close packing is reached, which is desirable for the final film homogeneity. For $c_{s i}>c_{s 2}$, physical drying induces a salt concentration increase able to instantaneously destabilize the suspension. Aggregates appear and sediment. The final film will be heterogeneous. Finally for $c_{s 1}<c_{s i}<c_{s 2}$ the trajectory reaches the percolation region III during drying. If the gel phase can be reorganized, physical evaporation may still permit reaching close packing but the final film homogeneity may not be perfect.

Note that the discussion above is only an illustration of the effect of physical drying on the chemical composition of the system, the latter being responsible for the onset of simultaneous chemical drying. In particular, the 
boundaries in the phase diagram of fig. 5 depend on the experimental time. Indeed, the percolated gel phase was observed in boehmite/cerium nitrate suspensions after a few hours. If a suspension with $c_{s 1}<c_{s i}<c_{s 2}$ is used in a drying process lasting only a few minutes, no gel phase will be produced. Indeed, the phase diagram is expected to be built at equilibrium and the discussion about trajectories in the phase diagram above implicitly assumes these trajectories are traveled along in a quasi-static manner. This description neglects important kinetic effects. In the next section, the role of one type of kinetic effect, namely hydrodynamic transport, is highlighted.

\subsection{The role of fluid flow}

In forced unidirectional drying, evaporation and mass conservation impose a simple uniform fluid flow that does not need modeling. In practice, drying is seldom unidirectional and often proceeds through front propagation. The initial colloidal suspension is one side of the front and a compact wet zone can be found on the other side. Evaporation on the large surface of the latter zone induces a significant lateral flow from the other side of the front. This flow brings colloids to the dense zone, allowing its growth and therefore the front shift [7]. In this front region, the dense layer assembly is flow-driven in a manner similar to what is observed in filtration. The role of evaporation itself is mainly to generate the hydrodynamic flow. In this $2 \mathrm{D}$ drying process, the dense layer assembly therefore involves a competition between stabilizing electrostatics and diffusion on one hand and destabilizing hydrodynamics and attractive colloidal forces on the other hand. A purely thermodynamic description is not sufficient anymore and the fluid flow needs to be modelled.

The general Eulerian description of suspension flows involves at least the colloid volume fraction field $\phi(\boldsymbol{x}, t)$, if one does not keep track of local microstructure changes. This quantity depends on the hydrodynamic flow through a locally averaged velocity field. Several equivalent descriptions are possible, using either the volume averaged fluid phase velocity (i.e., the pure solvent velocity) or the suspension averaged velocity (i.e., the volume averaged velocity of the mixture of solid particles and fluid solvent). Here we use the latter, denoted $\boldsymbol{u}(\boldsymbol{x}, t)$. For colloidal flows, the particle Reynolds and Stokes numbers are always much smaller than unity so inertia/acceleration terms can be neglected safely in the Navier-Stokes and Newton equations for the fluid phase and particles, respectively. In this context, general continuity and mass and momentum conservation equations can be derived rigorously for the suspension. The momentum conservation equation resembles the Stokes equation for a pure Newtonian fluid but an additional so-called "particle stress" is added to the usual stress [8]. A recent detailed analysis can be found in the work of Nott and coworkers [9]. These equations take the general form

$$
\begin{gathered}
0=-\nabla P+\nabla \cdot\left[2 \eta_{s} \boldsymbol{S}+\boldsymbol{\Sigma}^{p}\right], \\
0=\nabla \cdot \boldsymbol{u},
\end{gathered}
$$

$$
\frac{\partial \phi}{\partial t}+\boldsymbol{u} \nabla \phi=-\nabla \cdot \boldsymbol{j}^{p}
$$

where $P$ is some average pressure field imposed by the incompressibility condition (2), $\eta_{s}$ is the solvent viscosity, $\boldsymbol{S}$ is the average rate-of-strain tensor, $\boldsymbol{\Sigma}^{p}$ is the contribution of particles to the suspension stress, and $\boldsymbol{j}^{p}$ is a migration flux due to particle-particle and particle-fluid interactions. These equations still require closure relations. Here we use the classical suspension balance model [10-12] in the limit of slow variations of $\phi$ and $\boldsymbol{u}$ at the scale of particles and quasi-isotropic suspension microstructure. The latter condition is achieved if the hydrodynamic stress is dominated by the stresses due to Brownian motion colloidal interactions [13]. The particle stress $\boldsymbol{\Sigma}^{p}$ then reduces to a quasi-Newtonian contribution $2 \eta(\phi) \boldsymbol{S}$ plus an isotropic stress absorbed into the pressure. $\eta_{s}+\eta(\phi)$ is the effective suspension viscosity, where $\eta(\phi)$ is a correction depending in general on the collisions, interactions between particles. Still in the same conditions, the migration flux reduces to

$$
\boldsymbol{j}^{p} \simeq-D_{0} f(\phi) \frac{V_{p}}{k T} \nabla \Pi
$$

where $D_{0}$ is the colloid diffusion coefficient in the dilute limit, $f(\phi)$ is the sedimentation hindrance function (many-body mobility divided by the isolated colloid mobility), $V_{p}$ is the volume of one colloid, and $\Pi$ is the generalized osmotic pressure, or particle pressure, containing contributions both from colloidal and hydrodynamic forces. In the discussion below, we neglect the latter so $\Pi$ is considered close to the classical osmotic pressure and $\boldsymbol{j}^{p} \simeq-D(\phi) \nabla \phi$ where $D(\phi)=D_{0} f(\phi) \frac{V_{p}}{k T} \frac{\partial \Pi}{\partial \phi}$. To summarize, model (1)-(2)-(3) solves the fluid flow in the simplest manner, i.e. with a Stokes equations supplemented with only a concentration-dependent viscosity. The model accounts, however, for the influence of this flow and of colloidal interactions on mass transport. To illustrate the influence of the evaporation-induced flow on the 2D drying of a "simple" hard sphere suspension, model (1)-(2)-(3) is solved in a $2 \mathrm{D}$ rectangular geometry corresponding to the microfluidic pervaporation system used in [14]. The bottom and left sides are solid impermeable walls, the right side is a suspension inlet at constant concentration and the top side is a pervaporation surface letting out a uniform and constant solvent flux due to evaporation but blocking colloids. The streamlines of the $2 \mathrm{D}$ flow are represented in fig. 6(a) and fig. 6(b) for Péclet numbers $P e=L V_{E} / D_{0}$ equal to 1 and 10, respectively. Here the lengthscale $L$ is the film height and $V_{E}$ is the evaporation rate. The influence of the flow on the colloid volume fraction is illustrated in fig. 6(c) and fig. 6(d). For $P e<1$ colloids can diffuse against the driving flow so no skin is created and the concentration is mostly uniform in the cross section. For $P e>1$, advective transport dominates diffusion so a skin builds up along the evaporation surface and the concentration process subsequently remains two dimensional.

When the hard spheres considered so far acquire surface charges $Z$, their mutual repulsion increases and they can resist flow-driven assembly more intensely. This is illustrated in fig. 7 where it may be seen that uncharged 


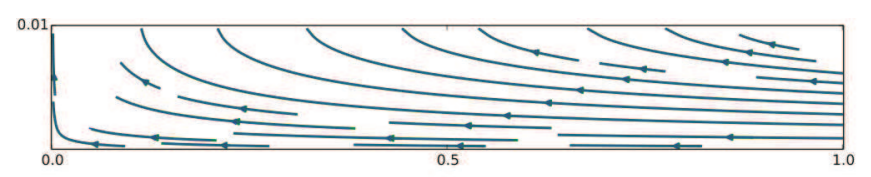

(a) Streamlines at $P e=1$.

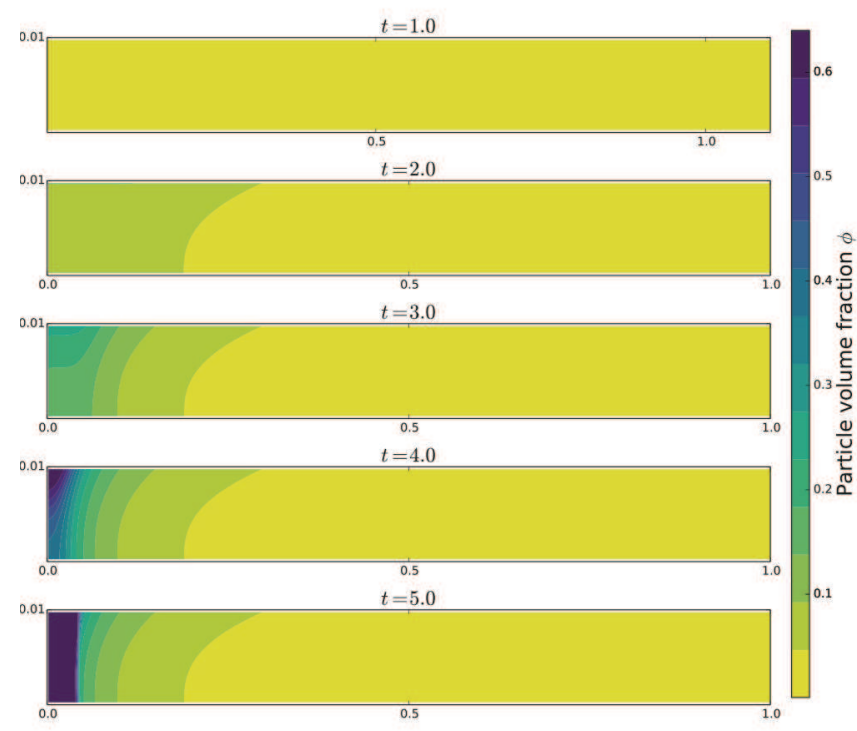

(c) Volume fraction at $P e=1$.

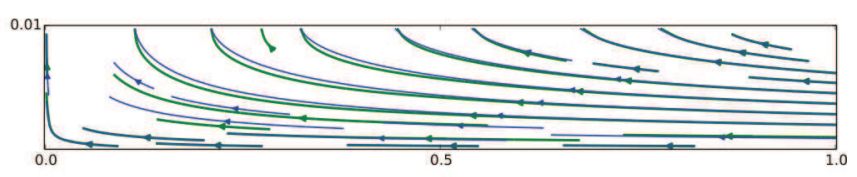

(b) Streamlines at $\mathrm{Pe}=10$.

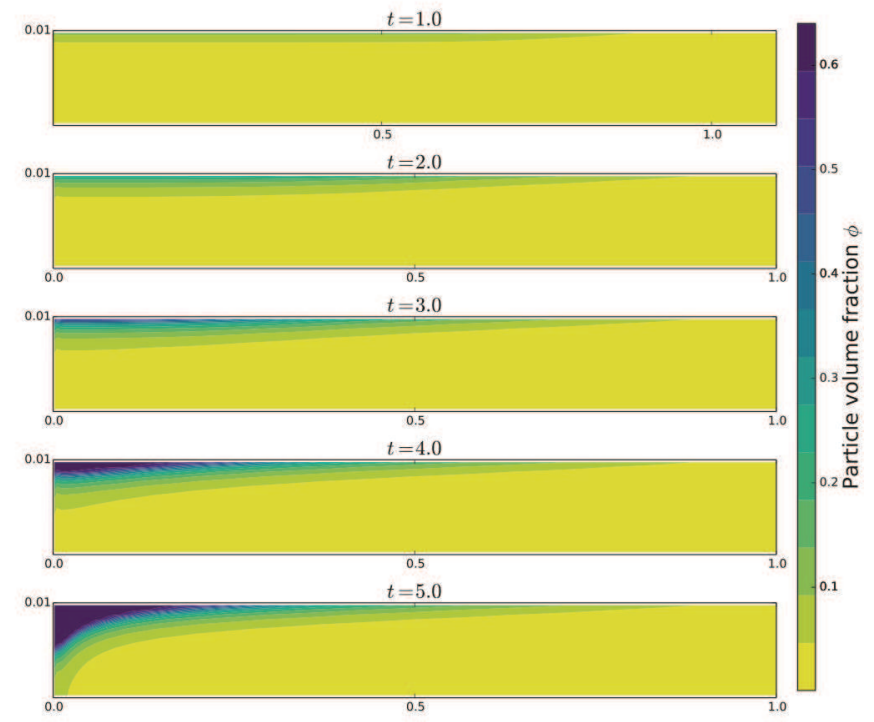

(d) Volume at $P e=10$.

Fig. 6. Hard sphere suspension $2 \mathrm{D}$ drying simulation in a pervaporator. In fig. 6(a) and (b), the blue (respectively, green) streamlines are results of computations with a constant (respectively, variable) viscosity. Figures 6(c) and (d) were obtained with a constant viscosity.

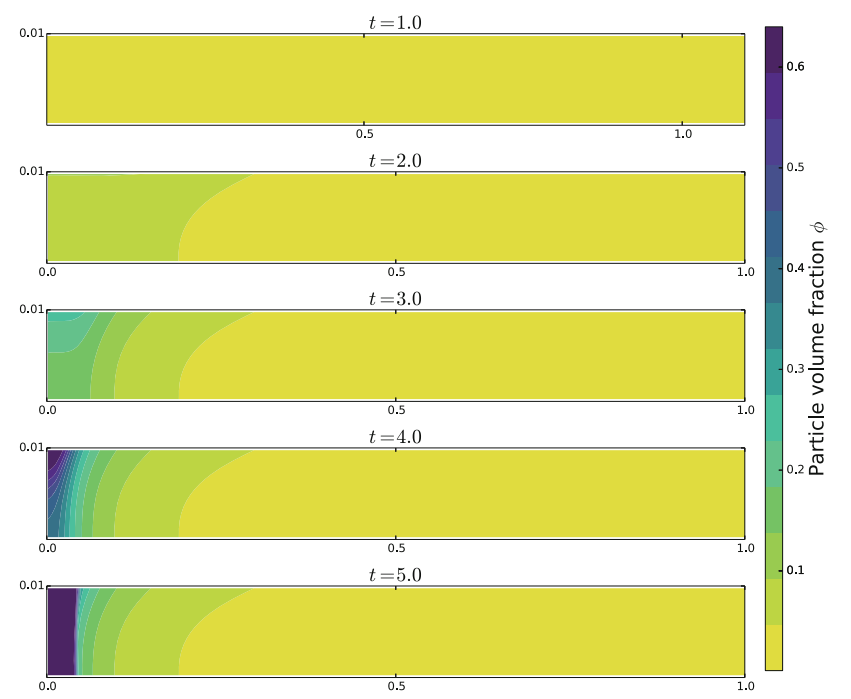

Fig. 7. Volume fractions snapshots obtained in simulations with increasing colloidal surface charges.

particles tend to nucleate a dense region in the upper left corner of the evaporator while this is partly prevented at intermediate surface charge and completely avoided at the higher surface charge.

These simulations were conducted at constant interaction range. Note that increasing the salt concentration, either intentionally or as a consequence of evaporation, would reduce this interaction range. For high salt concentrations and vanishing interaction ranges the solution would then be close to that of hard spheres. In a drying experiment, it is thus possible to avoid skin formation at initial times thanks to charge stabilization by adjusting the $p \mathrm{H}$ or the salt content, but to obtain a skin later on due to the increase of the average salt concentration induced by evaporation. Accounting for these effects is possible if the full dependence of the equation of state $\Pi\left(\phi, c_{s}, Z\right)$ is used in eq. (4). In this case the colloid flux is not $-D(\phi) \nabla \phi$ anymore. It depends also on gradients of salt concentration $\nabla c_{s}$ and surface charge $\nabla Z$. Simulations involving these terms require the additional resolution of averaged mass conservation equations for the ionic species (NernstPlanck equations).

\subsection{Conclusion}

The drying of charge-stabilized colloidal suspensions is made complex by to the coupling between physicochemistry and hydrodynamics. Indeed, the solvent evaporation flux at the free surface induces a physical drying of both particles and solutes, in particular ionic species determining the electrostatic interaction range. Even if the details of the hydrodynamic flow are neglected, physical evaporation can induce "chemical evaporation". If the 
hydrodynamic flow is important, it is influenced by colloids at least at the level of the effective suspension viscosity, but more complex effects can arise at high Péclet numbers. On the other hand, the colloid volume fraction field is coupled to hydrodynamics at least at the level of simple advective transport, which can induce skin formation or not, but once again more complex colloid fluxes can appear for particle Peclet numbers larger than unity due to colloid-colloid hydrodynamic interactions, like for example shear-induced migration. A faithful modeling of evaporation-induced assembly of colloid-based functional coatings requires a good knowledge of the suspension rheology and out-of-equilibrium thermodynamics, two active domains of research per se, and is thus a difficult fascinating challenge.

\section{On the interest of studying the drying behavior of food colloids at single droplet scale}

Drying is a well-established process to stabilize the biological and physico-chemical properties of food products during long-term storage, facilitate product handling and protect encapsulated materials in carrier matrices $[15,16]$. It consists for food liquid materials in spraying the feed solution into a cloud of droplets within a flow of hot air: the water is rapidly evaporated from the droplets, thus resulting in dried particles whose physical characteristics (eg, size distribution, shape and density) and typical aerodynamic behaviors determine their final application [17-19]. The ability of running spray drying on a large scale, with multiple possibilities to produce composite materials [20], makes it nowadays a widely spread technology in the food sector.

Nevertheless, the polydisperse feature of the flying droplets formed upon spraying comes with different drying kinetics in each droplet and finally with a more or less wide particle size distribution (fig. 8), making it difficult to model the transport phenomena and study the phase transition and resulting mechanical instability [21]. Moreover, the fast-drying kinetics clearly makes the experimental ability to monitor and sample the process in real equipment even more complex [22].

Single droplet experiments, usually in a pendant configuration, have been proposed to overcome these limitations, as they represent an easier way to study the drying of a given concentrated product in a controlled environment. The pendant configuration makes it possible to maintain a spherical shape as much as possible and avoid an inversion of the curvature of the particle, whereas the porous shell collapses under gravity and pressure gradient when water evaporates in the sessile configuration $[23,24]$. Moreover, the controlled boundary conditions and drying timescale, which usually stands for minutes instead of seconds, make it possible to record the drying kinetics through mass and length indicators. By recording the pendant droplet evolution with optical or fluorescence analytical techniques, rheological changes, skin formation and

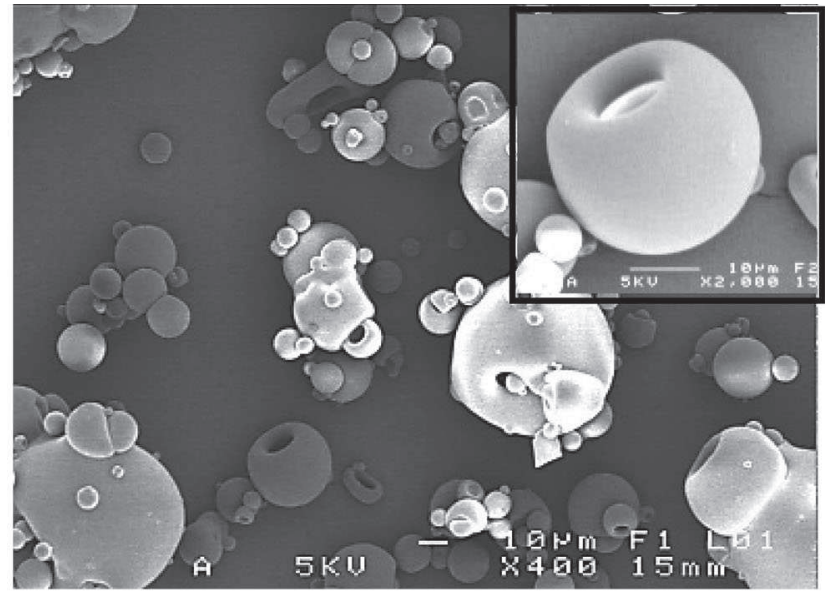

(a)

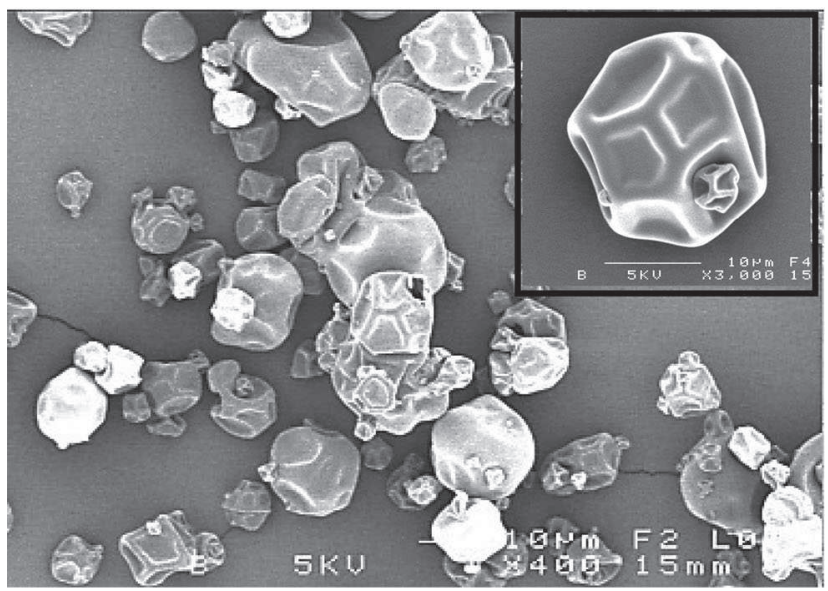

(b)

Fig. 8. Particle shapes of dairy proteins material. (a) Whey proteins isolate. (b) Casein micelles isolate.

physical deformations can be tracked. It allows a more direct, flexible and detailed investigation of the influence of specific ingredients on the particle characteristics. This experimental concept represents therefore a complementary approach to spray drying in order to improve the understanding of fundamental phenomena governing drying and further model these latter, that are not yet accessible on an industrial scale.

\subsection{Single droplet studies of dairy particle formation}

During the drying of concentrated solutions of polymer and/or colloid, it has been observed first the formation of a shell at the drop free surface coming from solutes accumulation due to the evaporative flux [24]. Depending on the chemical and physical properties of the solute and the external conditions, later on different surface instabilities occur such as buckling, invagination, delamination and cracks [25]. Sadek et al. [26-28] investigated on the drying dynamics of pendant droplets made of the two main milk proteins, whey proteins and casein micelles. 

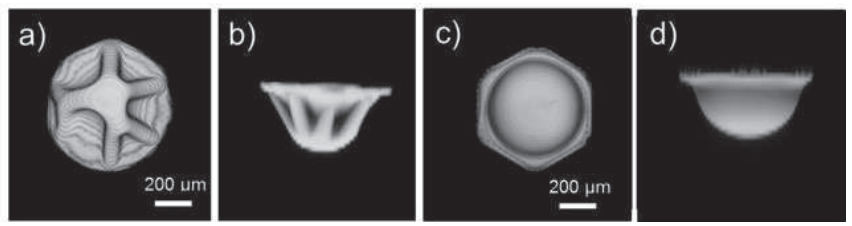

Fig. 9. 3D confocal reconstitutions of final single pendant particles shape of casein micelles $((\mathrm{a}),(\mathrm{b}))$ and of whey proteins $((\mathrm{c}),(\mathrm{d}))$, top and profile views, respectively.

For that purpose, the concentrated protein solution drop was hanged to a micropatterned substrate and exposed to stressful conditions (relative humidity, $R H$, below $5 \%$ ). For both milk proteins, the drying of the single pedant droplet revealed three similar stages involving different evaporation rates and droplet dynamics, that were thus reached at different time according to the protein type. At the beginning, the droplet behaved as a pure liquid without a significant influence of the protein surface activity on the drying rate, that remained diffusion controlled. Next, a sol-gel transition was believed to occur at an average concentration around $41 \mathrm{wt} \%$ and $15 \mathrm{wt} \%$ for whey proteins and casein micelles, respectively. The gelation fixed the surface area while the water was still evaporating, inducing the buckling (initial surface instability) of the droplet and revealing a concomitant hydric rather than mechanical equilibrium. Indeed, the surface instability was believed to be mainly initiated by rheological changes here, as it did not impede at first the loss of mass (water evaporation). The skin then progressively thickened into a homogeneous protein shell, leading to the formation of an internal vacuole. Once the skin layer was formed, the surface underwent surface instabilities such as buckling and invagination according to the protein kind, which lead to typical and reproducible particle shapes. Smooth, hemispherical and hollow particle was obtained for the drying of the single droplet of whey proteins, whereas deflated wrinkled particle shape resulted from the drying of the single droplet of casein micelles (fig. 9). These results suggest particular mechanical properties of the milk protein shell leading to a predictable and a characteristic particle shape, regardless of the experimental configuration. Indeed, Bouman et al. [29] investigating the drying of a single sessile droplet of whey proteins at different drying temperatures, reported the same droplet dynamics and vacuole nucleation occurrence. On the other hand, $\mathrm{Fu}$ et al. [30] reported surface invagination similar to that observed for casein micelles during the drying of single suspended droplet of whole and skimmed milk that was thus mainly constituted by casein micelles.

Moreover, the mechanical properties of the final materials of proteins were characterized using microindentation testing by Sadek et al. [27]. It was shown that the interface of micellar casein micelles reached an earlier sol-gel transition, followed by elastic and plastic regimes in which the shell distorted and buckled to form a final wrinkled particle. On the opposite, the interface of whey proteins became elastic only half the drying time, keeping a spherical shape, which finally fractured at the

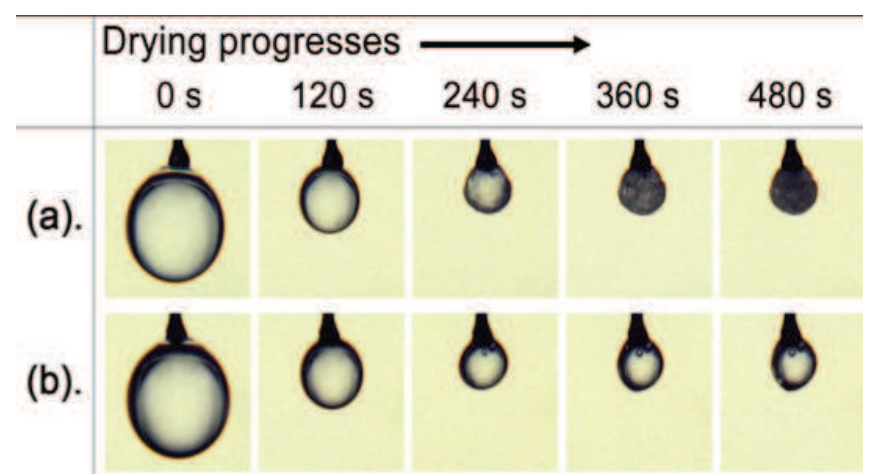

Fig. 10. Single droplet drying processes: (a) lactose solution; (b) sucrose solution ((a) shows the opacity has developed which is well known due to the lactose transformation towards crystallization becoming much less water soluble).

end of drying. The mechanical difference between the two plastic shells might thus be explained by the behavior of proteins in jamming conditions. Interestingly, the same authors showed that the particle shape was governed by the major protein type when drying whey protein: casein micelles droplets, mostly spherical for major whey protein mix, and mostly wrinkled particles for major casein micelles mix [31]. Interestingly, these specific particle morphologies were similar to those observed for single pendant droplet experiments, and for powders obtained at real industrial scale spray drying, regardless of the considerable differences in drying timescale and temperature.

\subsection{Drying modeling based on single droplet experiment}

On the other hand, single drop experiments have been widely used to establish the kinetics of drying and the parameters fed to the milk drying modeling of one-, twoand three-dimensional nature. These models, taking into account both drying kinetics and drying temperatures, have been mostly developed using a pendant configuration glass-filament method with some improvements [32] in order to predict the final particle characteristics including moisture content $[22,33]$. Figure 10 shows a single droplet hung on a glass filament tip during such drying experiments.

The droplet drying experiments may be conducted under isothermal air conditions or transiently varying temperature conditions as long as both the weight loss and the temperature of the droplet recorded. The following outlines the obtainment of the drying kinetics through a Reaction Engineering Approach (REA) [34,35]. The same authors [36] have summarized a wide range of the REA kinetics parameters for food materials. In particular, for several typical dairy products, drying kinetics parameters have been obtained [37-40].

The Reaction Engineering Approach (REA) is a semiempirical model that defines evaporation as an activation energy that needs to overcome the energy obstacle in order to initiate the process of moisture removal. This approach characterizes the driving force for moisture removal at the 
solid-gas interface, i.e. droplet-air interface, through vapor density difference, as expressed in the following equation:

$$
\frac{\mathrm{d} m}{\mathrm{~d} t}=-h_{m} A\left(\rho_{v, s}-\rho_{v, b}\right)
$$

where $\mathrm{d} m / \mathrm{d} t$ represents the drying rate $\left(\mathrm{kg} \cdot \mathrm{s}^{-1}\right), h_{m}$ the mass transfer coefficient $\left(\mathrm{m} \cdot \mathrm{s}^{-1}\right)$, A the surface area of the droplet $\left(\mathrm{m}^{2}\right)$ and $\rho_{v, s}$ and $\rho_{v, b}$ represent the vapor density $\left(\mathrm{kg} \cdot \mathrm{m}^{-3}\right)$ at the droplet surface and bulk air, respectively. Since the surface vapor density $\rho_{v, s}$ will constantly vary throughout the drying process, it could then be expressed as

$$
\rho_{(v, s)}=\psi \rho_{v, s a t}\left(T_{s}\right),
$$

where $\psi$ denotes a fractionality coefficient in relation to the moisture content of the droplet and $\rho_{v, s a t}\left(T_{s}\right)$ denotes the saturated surface vapor density and this term is a function of the surface temperature $T_{s}(\mathrm{~K})$. The distribution of temperature of a solid body can be captured through the Biot number. This last represents the competition between the thermal energy transfer fluxes inside and outside the solid body, namely the liquid droplet in our case. Previous study has shown that the drying-based Biot number was small when tested under several evaporation conditions, meaning that the droplet surface-centre temperature difference is negligible and thus $T_{s}$ reasonably equates to the average droplet temperature $T_{d}(\mathrm{~K})$. The fractionality coefficient $\psi$ also represents the relative humidity or water activity at the droplet-air interface. In REA modeling, the apparent activation energy of evaporation, represented by $\Delta E_{v}\left(\mathrm{~J} \cdot \mathrm{mol}^{-1}\right)$ describes the change of $\psi$ during moisture removal from the droplet using the subsequent correlation:

$$
\psi=\exp \left(-\Delta E_{v} /\left(R T_{d}\right)\right)
$$

where $R$ is the universal gas constant $\left(8.314 \mathrm{~J} \cdot \mathrm{mol}^{-1}\right.$. $\mathrm{K}^{-1}$ ) and $T_{d}$ represents the average droplet temperature. By substituting (7) into (6) and rearranging the terms, the apparent activation energy term $\Delta E_{v}$ can be expressed as

$$
\Delta E_{v}=-R T_{s} \ln \left(\rho_{v, s} / \rho_{v, s a t}\left(T_{s}\right)\right) .
$$

Equation (8) indicates that $\Delta E_{v}$ is a reflection of a vapor concentration depression at the interface of the droplet. By rearranging (8) while substituting the term $\rho_{v, s}$ into (5), one can obtain:

$$
\Delta E_{v}=-R T_{d} \ln \left(\left(-\frac{\mathrm{d} m}{\mathrm{~d} t} \frac{1}{h_{m} A}+\rho_{v, b}\right) / \rho_{v, s a t}\right) .
$$

In order to simulate the apparent activation energy $\Delta E_{v}$ at a given drying condition, three parameters such as $\mathrm{d} m / \mathrm{d} t, T_{d}$ and $A$, need to be experimentally determined. $\Delta E_{v}$ can be normalized by using the equilibrium or maximum activation energy $\Delta E_{v, b}$, which yields the relative activation energy $\Delta E_{v} / \Delta E_{v, b}$ (value ranges between 0 and 1 ). When the apparent relative humidity is in equilibrium with the bulk air relative humidity, it means that the apparent activation energy $\Delta E_{v}$ has also achieved its equilibrium $\Delta E_{v, b}$. At this equilibrium point, the rate of droplet mass loss is zero whilst the droplet temperature and moisture content match those of the drying medium. Hence the maximum or equilibrium activation energy $\Delta E_{v, b}$ can be calculated from the equation below:

$$
\Delta E_{v, b}=-R T_{b} \ln \left(\rho_{v, b} / \rho_{v, s a t}\left(T_{b}\right)\right),
$$

where $T_{b}$ represents the bulk air temperature $(\mathrm{K})$ and $\rho_{v, \text { sat }}\left(T_{b}\right)$ represents the saturated vapor density of the bulk air $\left(\mathrm{kg} \cdot \mathrm{m}^{-3}\right)$.

Previous investigations have determined that the relative or normalized activation energy $\Delta E_{v} / \Delta E_{v, b}$ can be correlated with the droplet's moisture content $X-X_{b}$, generating a master characteristic curve of relative activation energy for a given material:

$$
\frac{\Delta E_{v}}{\Delta E_{v, b}}=f\left(X-X_{b}\right)
$$

where $X_{b}$ and $X$ are termed as the particles equilibrium moisture content $\left(\mathrm{kg} \cdot \mathrm{kg}^{-1}\right.$, dry basis) and moisture content $\left(\mathrm{kg} \cdot \mathrm{kg}^{-1}\right.$, dry basis), respectively. This empirical equation has been tested under many drying conditions and was found to correlate well with the drying kinetics of material with known initial solids content. In other words, this equation is the "fingerprint" of a material's drying behavior. These kinetics data can be implemented into simulation programs home-made $[41,42]$ or commercially available computational fluid dynamics modeling software with specific user established operating files for scale up studies and industrial spray dryer design [43-46].

\section{Thin cosmetic films structuring due to phase separation induced by drying}

The drying of complex solutions, composed of a polymer or a mixture of polymers dissolved in a mixture of solvents, is a problem of great scientific and technical interest due the diversity of physical phenomena that are implemented and to the great variety of resulting applications. We meet this process, for example, in cosmetology, where complex polymer-solvent solutions are normally used in the form of thin films, a few $\mu \mathrm{m}$ to a few hundred $\mu \mathrm{m}$ thick, spread on a support such as skin. This case involves bio-compatible polymers dissolved in water, a fundamental solvent in biophysics. Additional additives can be used, such as preservatives or antibacterial agents to prevent the proliferation of microorganisms in the solutions, or additives that can restructure the film by modifying the interactions with water during drying.

There are two main aspects of the drying of thin films that interest the scientist, the industrialist and the consumer. The first is the kinetics of drying, which must be controlled reproducibly according to the application envisaged, such as hydration of skin (creams), delivery of bactericidal and virucidal molecules (hydro-alcoholic gels) or therapeutic molecules (ointments), or controlled hydration of plants (moisturizing gels). The structuring or morphology of the dry film is another aspect of fundamental interest: the morphology of the film can undergo significant 
changes during drying, such as the formation of blisters or craters, accumulation of matter or tearing.

These two aspects are not a priori independent. For example, rapid dehydration of a thick film may result in the formation of a skin on the free surface of the solution [47]. Under these conditions, the evaporation of solvents into the ambient atmosphere, and thus the kinetics of drying, are affected, as well as the final state of the film that will then contain a significant fraction of residual water. An accurate description of these two aspects requires taking into account many of the physical processes that can arise not only within the film - diffusion and convection of material and thermal energy, a glass transition and viscoelastic relaxation - but also at the interface between the film and the ambient atmosphere - evaporation, demixing, formation of skin - and that between the film and its support - capillarity, wettability and pinning of the triple line.

It is therefore important to combine experimentation and modeling in order to reach a realistic physical description of both the kinetics of drying and the structuring of thin films used in cosmetology.

Several physical phenomena related to evaporation, can lead to a structuring of thin films. In low-viscosity colloidal suspensions, pinning of the triple line can induce convective motions within the film, leading to irregular accumulation of material similar to that observed during evaporation of drops of coffee (coffee rings [48,49]). Evaporative cooling of a solvent in the presence of a humid atmosphere can cause nucleation of fine water droplets that are structured on the surface of the film by thermocapillary convection and then penetrate the film, giving it an ordered microstructure after complete evaporation [50]. The phenomenon of phase separation within a complex solution is also a potential pathway for structuring of thin films. This phenomenon is commonly used in the synthesis of porous polymer membranes and foams, for example. Phase separation can be induced in this case by rapidly cooling the solution (thermal-induced phase separation: TIPS), or by quenching after spreading on a support and partial evaporation of the solvent [51]. The process of aircasting is another interesting approach that consists in dissolving the polymer in a mixture of solvents of which one is very little volatile. Evaporation of the more volatile solvent then decreases the solubility of the polymer and induces structuring by phase separation $[52,53]$.

In each of the two cases envisaged above, the solvent evaporation phase plays a fundamental role in the production of membranes with anisotropic structure highly sought after for ultra-filtration - by accumulating the polymer material inside the film. This approach is, for example, implemented during the formation of porous membranes from a binary solution of cellulose acetate and acetone [54].

It is also possible to use the process of phase separation induced by drying to structure aqueous solutions used in cosmetology. The colloidal suspensions are spread in the form of a thin film ( $\mathrm{TF}$ ) for which the evaporation of water completely determines its evolution. In this case, the aqueous solution is generally composed of a watersoluble biocompatible polymer $(\mathrm{P})$ such as sodium car- boxymethylcellulose or polyvinyl alcohol, dissolved in water $(\mathrm{W})$ in the presence of a small fraction of a preservative or structuring agent $\left(\mathrm{X}_{\mathrm{W}}\right)$ very little volatile, partially soluble in $\mathrm{W}$ and incompatible with $\mathrm{P}$ : $\mathrm{TF}=\mathrm{P}+\mathrm{W}+\mathrm{X}_{\mathrm{W}}$. In the case of films used in cosmetics, only the solvent is notably volatile. Drying can induce interesting behavior in these ternary systems at the morphological level. The initial mass fraction $w_{\mathrm{W}}^{0}$ implemented for this type of film is of the order of $95 \%$, making it possible to obtain an initially homogeneous solution whose structuring results from separation processes during drying of the thin film.

\subsection{Structuring of a cosmetic thin film}

Under certain conditions of composition and drying, one can observe a progressive structuring of cosmetic aqueous films due to phase separation induced by water evaporation. Figure 11 shows binocular microscope figures tracking the structuring of a cosmetic film during drying. The structuring agents $\mathrm{X}_{\mathrm{W}}$ that we have studied are phenol, phenoxyethanol and phenoxyisopropanol, common additives used in cosmetology and pharmacology. Other agents were also considered - ethanol, glycerol and butanolbut these did not lead to any structuring of the film, because they are either totally miscible with water (ethanol and glycerol) or too wetting to form drops within the film (butanol). We describe here the results obtained with phenoxyethanol, whose limit of miscibility in pure water is 2.4 wt. $\%$ at $20^{\circ}$.

At the initial time $t_{0}$, the solution is spread mechanically on a very clean microscope slide, which is placed in an environmental chamber allowing precise control of the drying conditions. The newly formed thin film is homogeneous and initially has no deformation. As soon as drying begins, even blisters form within the film in the immediate vicinity of the free surface (see fig. 11). The time $t_{d}$ when all the first blisters have formed depends first on the evaporation rate, $V_{E}$, i.e., the mass flow of water $\phi_{m}$ leaving the film, and secondly on the initial mass fraction of $\mathrm{X}_{\mathrm{W}}$ and its chemical nature (see previous discussion). For a relative humidity $a_{\mathrm{w}, v}^{\infty}=0.42$ and a typical drying temperature $T_{\infty}=20^{\circ} \mathrm{C}, t_{d}$ varies over a few seconds for an initial mass fraction $w_{\mathrm{X}}^{0}=2.4 \%$ to a few minutes for low values of $w_{\mathrm{X}}^{0}$ (see fig. 12).

We also observe that the blisters partially coalesce and deform as the film loses solvent (see insert at $t_{0}+23 \mathrm{~min}$, fig. 11), because of the increasing mechanical constraints exerted by the film on the blisters as drying proceeds. Once the film is dry, it exhibits a permanent structure in the form of craters with small diameters (of the order of a few $\mu \mathrm{m}$ ) with a relatively homogeneous distribution. These craters occupy the places of the blisters that had appeared during drying (see inset at $t_{0}+50 \mathrm{~min}$, fig. 11).

We have established [55] that the structuring of cosmetic aqueous films could originate in the demixing of the agent $\mathrm{X}_{\mathrm{W}}$ with the water present in the film. This phase separation occurs when the mass fraction $w_{\mathrm{X}}$ reaches the solubility limit of $\mathrm{X}_{\mathrm{W}}$ in the film. The modeling of the kinetics of drying that we present in the next section con- 

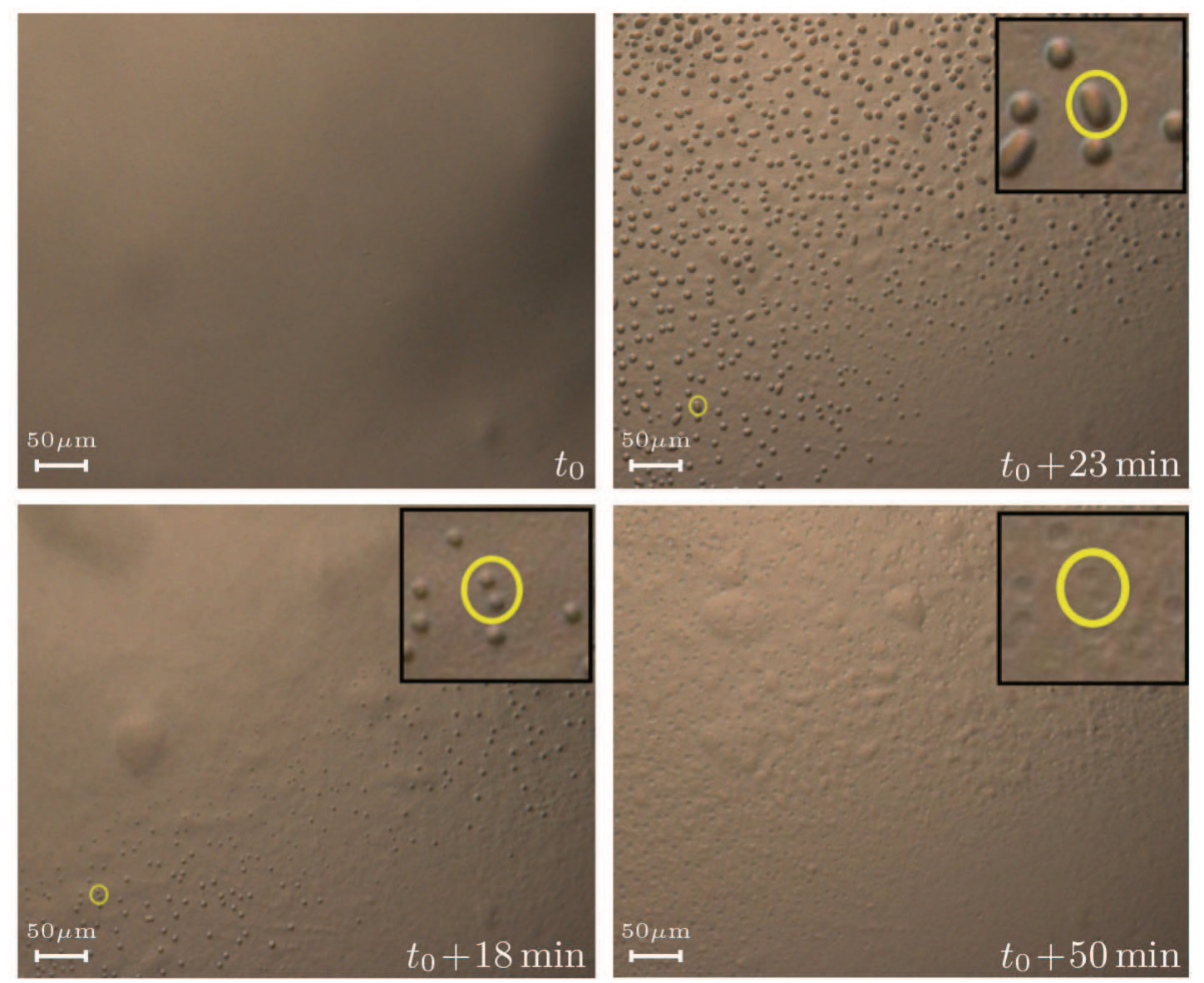

Fig. 11. Binocular microscopy images taken at different times, typical of the temporal evolution of structuring of a cosmetic thin film by phase separation induced by evaporation. At the initial time $t_{0}$, the film that has just been spread on its support, is homogeneous and shows no significant deformation. At the final time $t_{f}=t_{0}+50 \mathrm{~min}$, the film is completely dry and exhibits a structuring in the form of craters replacing the blisters, as shown by the insets at $t_{0}+18 \mathrm{~min}$ and $t_{0}+50 \mathrm{~min}$.

firms to a good approximation the values of the time of appearance $t_{d}$ of the first drops.

\subsection{Modeling of the drying kinetics}

Combining experimental and theoretical studies of the drying kinetics of an aqueous thin film of TF type is of paramount importance in cosmetology and pharmacology. The evolution of the morphology of the film during the drying can have dramatic consequences for the practical implementation of these films. Kinetic studies can provide information on the various interactions not only between the constituents of the mixture but also between the solution and its environment (evaporation atmosphere and spreading support). Understanding the mechanisms of drying and structuring of an aqueous thin film composed of water-soluble polymers requires a preliminary study detailed of the kinetics of drying, combining experiments and modeling.

\subsubsection{Experimental approach}

From an experimental point of view, the drying kinetics of a solvent-polymer thin film is generally studied from a direct or indirect gravimetric monitoring of the film throughout the duration of the solvent(s) evaporation phase. The volatility of the polymer suspended in the film can be assumed to be negligible compared with that of the solvent(s). The polymer therefore remains present in the solution until the end of the process and gradually becomes structured to finally take the form of a thin solid film. We mainly consider here the case of thin cosmetic films with an initial thickness $h_{0} \leqslant 100 \mu \mathrm{m}$.

Direct gravimetric monitoring of the drying kinetics by weighing the film is a relatively common approach and easy to implement. However, it is essential in this case to precisely control the various environmental parameters of drying: the temperatures of the surrounding air $T_{\infty}$ and of the support $T_{s}$, the ambient humidity, the state of the support surface and the flow velocity $v_{\infty}$ of the surrounding air in the vicinity of the film. It is therefore almost indispensable to work in a controlled atmosphere, in an enclosure where the physical parameters are measured continuously using various miniature sensors placed 


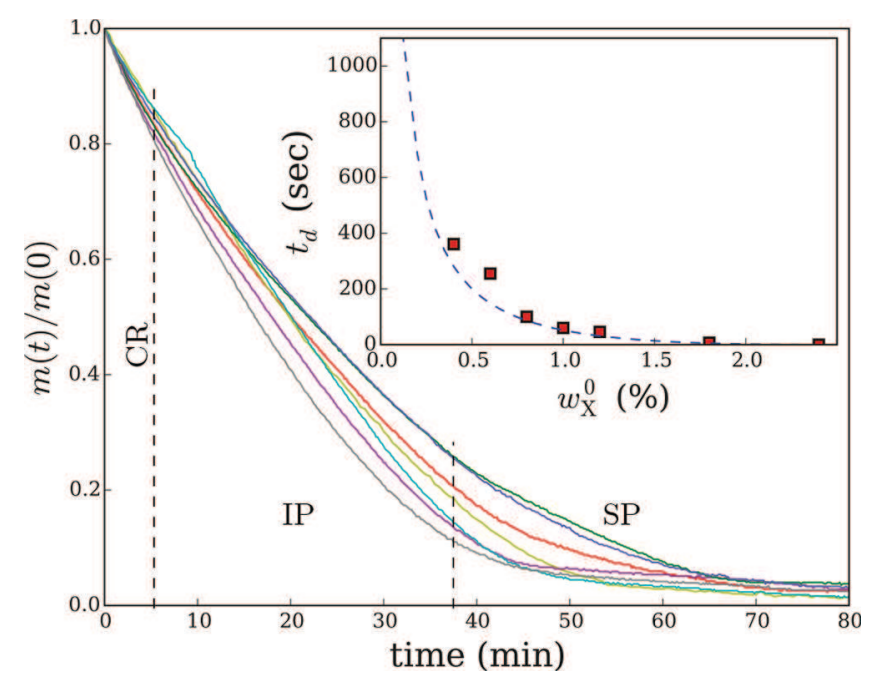

Fig. 12. Typical drying curves of aqueous films initially having a $w_{\mathrm{P}}^{0}$ mass fraction of $3 \%$ for different initial mass fractions $w_{\mathrm{X}}^{0}$, ranging from $0.4 \%$ to $2.4 \%$ (adapted from [55]). The inset shows times $t_{d}$ when the first drops appear in the film. The dashed line corresponds to the measurements, while the symbols (squares) correspond to the calculations. Three successive phases can be identified in the drying kinetics, denoted CR, IP and SP.

near and far from the film. The relative humidity $a_{\mathrm{w}, v}^{\infty}$ of the surrounding atmosphere can be imposed using various saturated saline solutions, for example. In order to minimize the time between the physical preparation of the film (for example, by spreading on the desired support) and its positioning on the precision balance, the spreader is placed in the hermetic environmental enclosure.

Depending on the initial thickness $h_{0}$, of the composition $\left\{w_{i}^{0}\right\}$, where $w_{i}$ is the mass fraction of the ith constituent of the mixture, and the initial shape of the film, the drying kinetics can exhibit large variations in the dynamics (drying time), which may require special experimental precautions. In the case of thick samples (several $\mathrm{cm}$ ), requiring several weeks for complete evaporation of the solvent, it is possible to manipulate the sample during drying without noticeably distorting the gravimetric monitoring [56]. It is quite different in the drying of thin films, whose dynamics can be only a few tens of minutes (see fig. 12). It is then impossible to envisage any manipulation of the film during drying and furthermore it is necessary to have a sufficiently sensitive and fast weighing method to be able to monitor the evolution of the mass $m(t)$ of the film with adequate accuracy. Several routes can be considered in this case: a high-precision gravimetric monitoring [55], thermogravimetric analysis (TGA) or differential scanning calorimetry (DSC) [57].

\subsubsection{Modeling of the drying kinetics}

Taking account of the measurements provided by the various sensors in the environmental chamber leads to a form of inverse analysis. This consists of validating microscopic models of water transfer based on average macroscopic quantities, such as monitoring the evolution of the mass of the sample as a function of time:

$$
m(t)=\sum_{i} \int_{V(t)} w_{i}^{c}(M, t) \mathrm{d} \tau,
$$

where $w_{i}^{c}(M, t)$ is the mass concentration of the species $(i)$ in the film, averaged over the entire volume $V(t)$ of the film. It can also be interesting to access the evolution of the temperature $T_{h}(t)$ and the average humidity $a_{\mathrm{w}, v}^{h}(t)$ of the film surface, which usually control the evaporation of the solvent towards the atmosphere. Depending on the physical properties of the film (initial dimensions, composition, homogeneity, interactions between its constituents, temperature, dynamic viscosity, elasticity, surface tension and thermal conductivity), the support (surface condition and temperature) and the surrounding atmosphere (convection, temperature and relative humidity) one can observe a very wide variety of drying behaviors, which manifest mainly by very different kinetics and film structuring. Thus, a film with low viscosity spread on a hydrophobic support will tend to deform and dry like a drop, while a viscous film spread over a wetting support will tend to dry like a flat film. In the latter case, if the triple line is pinned to the support, the lateral dimensions $L_{i}$ of the film plane can be assumed to be constant. Moreover, if these dimensions are large compared with the thickness $h(t)$ of the film, a one-dimensional modeling is generally sufficient.

Given the high initial viscosity of the suspension (nearly $10^{5}$ times that of water), because of the presence of the polymer, we can completely neglect convection within the film [58]. The drying kinetics shown in fig. 12 is therefore described well by an $1 \mathrm{D}$ isothermal (thin film, thus low Biot number and evaporative cooling neglected) evaporation-diffusion model. This model provides the spatio-temporal evolution of the mass concentration $w_{i}^{c}(z, t)$ of each constituent $(i)$ in the film [55] by solving the coupled equations of particle diffusion:

$$
\partial_{t} w_{i}^{c}=\partial_{z}\left(D_{i} \partial_{z} w_{i}^{c}\right)
$$

where $D_{i}$ is the diffusion coefficient of component $(i)$ in the film, which depends heavily on the composition of the film at time $t$. These equations are accompanied by the following boundary conditions: impenetrable support at $z=0$, whence

$$
\partial_{z} w_{i}^{c}(0, t)=0, \quad \forall i \in\left\{\mathrm{P}, \mathrm{W}, \mathrm{X}_{\mathrm{W}}\right\}
$$

and jump conditions (jump mass balance) at the mobile interface $z=h(t)$ for the non-volatile species:

$$
w_{i}^{c}(h(t), t) \dot{h}=-D_{i} \partial_{z} w_{i}^{c}(h(t), t), \quad \forall i \in\left\{\mathrm{P}, \mathrm{X}_{\mathrm{W}}\right\} .
$$

And for the solvent [59]:

$$
w_{\mathrm{w}}^{c}(h(t), t) \dot{h}+D_{\mathrm{w}} \partial_{z} w_{\mathrm{w}}^{c}(h(t), t)=-h_{m}\left(\rho_{\mathrm{w}, v}^{h}-\rho_{\mathrm{w}, v}^{\infty}\right)
$$

where $h_{m}$ is the mass transfer coefficient; $\rho_{\mathrm{w}, v}^{h}$ and $\rho_{\mathrm{w}, v}^{\infty}$ represent the water vapor density at the free interface and 
far from the film, respectively. By modeling the water vapor as a perfect gas, we can write $\rho_{\mathrm{w}, v}^{z}=a_{\mathrm{w}, v}^{z} \rho_{\mathrm{w}, s a t}^{z}$ where $a_{\mathrm{w}, v}^{z}$ and $\rho_{\mathrm{w}, s a t}^{z}$ are the water vapor activity and saturated density, respectively, for the position $z \geqslant h$. It remains finally to impose the conservation of the mass of the evaporated solvent:

$$
\dot{h}=-\bar{v}_{\mathrm{w}} h_{m}\left(\rho_{\mathrm{w}, s a t}^{h} a_{\mathrm{w}, v}^{h}-\rho_{\mathrm{w}, s a t}^{\infty} a_{\mathrm{w}, v}^{\infty}\right)
$$

where $\bar{v}_{\mathrm{w}}$ is the specific volume of the liquid water.

The differential equations (13) and (17) accompanied by the conditions (14), (15) and (16) constitute a nonlinear problem, whose numerical resolution can be challenging if we seek to describe the totality of the drying kinetics represented in fig. 12. A preliminary analysis of the experimental drying curves, however, makes it possible to apply common laws of behavior.

Typically, we observe (see fig. 12) three distinct successive phases in the drying of cosmetic aqueous films with a high initial fraction of water $\left(w_{\mathrm{W}}^{0} \geqslant 0.9\right)$. The drying always starts with a phase with quasi-constant evaporation rate $(\mathrm{CR})$. This type of behavior is also encountered in the drying of hydrogels, fruits and food $[35,56,60,61]$. This first phase usually lasts about five minutes under the drying conditions that we have implemented (room temperature and relative humidity close to $43 \%$ ). During CR, the evaporation of the solvent at the free surface of the film completely governs its loss of water. During this phase the mass fraction of water in the film remains sufficiently large that we can consider that the chemical activity of the water at the interface $a_{\mathrm{w}, v}^{h}$ stays close to one. The polymer $\mathrm{P}$ is therefore practically chemically not involved in the kinetics and the film dries almost like a pure water film during this phase. The second phase (IP), which then takes place for several tens of minutes, sees the chemical activity of the solvent decrease sharply because of the increase in the polymer mass fraction due to evaporation. The water loss results from a competition between surface evaporation and mass transfers in the volume of the film. This phase is in general strongly nonlinear because of the dependence of several physical quantities (diffusion coefficients and chemical activity of water at the interface) with the fraction mass of water. Interactions between the different constituents of the complex aqueous solution vary greatly during IP. We then observe a final phase (SP) during which the rate of evaporation tends to zero. This phase, corresponding to solidification of the cosmetic film, can be very complex and generally presents a challenge to an evaporation-diffusion modeling.

Despite the low initial mass factions $w_{\mathrm{X}}^{0}$ of the additive $\mathrm{X}_{\mathrm{W}}$, we observe that the blisters are always formed during the first CR phase, in the immediate vicinity of the free surface of the film. This observation reflects the difficulty of diffusion of $\mathrm{X}_{\mathrm{W}}$ through the film and a trend towards accumulation at the free surface. The demixing of $\mathrm{X}_{\mathrm{W}}$ is described well by the diffusive model presented previously, with a diffusion coefficient $D_{\mathrm{X}}$ which can to a good approximation be assumed constant during the short-lived CR phase. The values of the diffusion coefficient $D_{\mathrm{X}}$ and solvent flow $\phi_{m}$ can be determined from the experimental

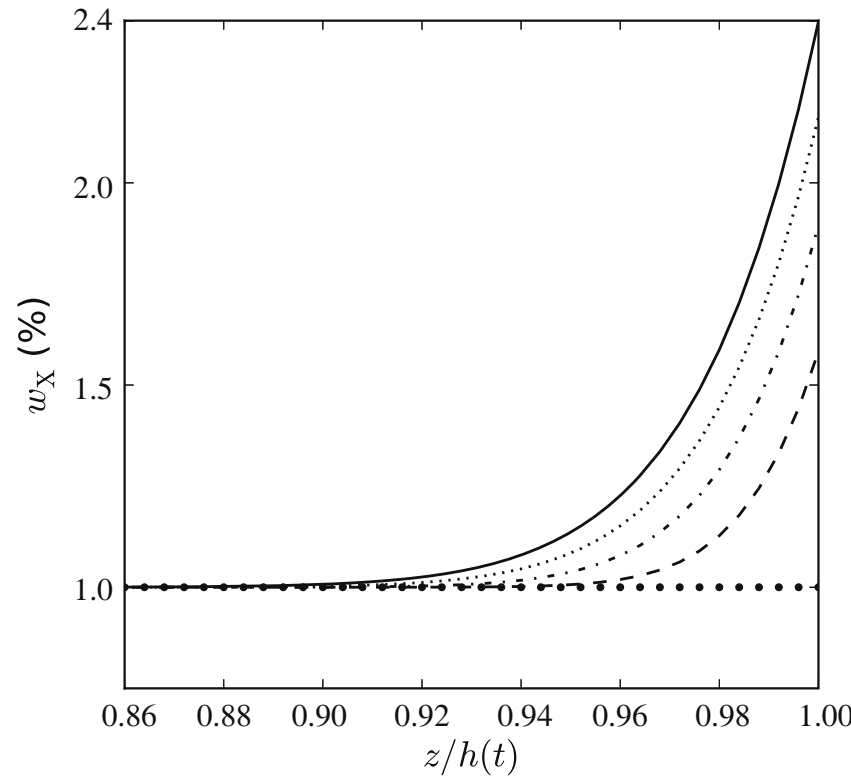

Fig. 13. Profiles of the mass fraction $w_{\mathrm{X}}$ of $\mathrm{X}_{\mathrm{W}}$ in the vicinity of the film interface $z / h(t)=1$ at different times $(t=0,13$, $26,39$ and $52 \mathrm{~s})$. The demixing takes place at $z / h(t)=1$, when $w_{\mathrm{X}}$ reaches the solubility limit, here $t \approx 52 \mathrm{~s}$. The initial mass fraction is taken to be $w_{\mathrm{X}}^{0}=1 \%$. (Figure adapted from [55].)

curves of drying shown in fig. 12. This allows a numerical resolution of eq. (13) with the boundary conditions (14) and (15), for $i \equiv \mathrm{X}$. We thus obtain the $\mathrm{X}_{\mathrm{W}}$ mass fraction profiles shown in fig. 13 and the demixing times represented in the inset of fig. 12. There is a good correspondence between the experimental results (dashed curve in the inset) and the proposed model (square symbols), which validates the evaporation-diffusion model chosen to describe the kinetics of cosmetic aqueous films.

\subsection{Crater formation}

The proposed evaporation-diffusion model makes it possible to predict the appearance of demixing within the cosmetic film during the drying cycle. On the other hand, it does not explain why this phase separation leads to formation of blisters and finally leaves craters in the dry film.

Analysis of the surface of the film by micro-confocal Raman spectroscopy shows that the blisters are predominantly composed of water. Once the film is dry, the Raman spectra show that each crater contains only a small amount of $\mathrm{X}_{\mathrm{W}}$. These observations imply that the blisters are composed of a thin film of $\mathrm{X}_{\mathrm{W}}$, which encloses at the time of demixing a micro-drop of water. This very small amount of water is protected from evaporation by a film of $\mathrm{X}_{\mathrm{W}}$, whose saturated vapor pressure (4 $\mathrm{Pa}$ in the case of phenoxyethanol) is very low compared with that of water. The viscosity of the solution greatly increases during drying, so the drop remains permanently in the immediate vicinity of the free surface. The water retention is maintained until the protective film breaks because of the strong mechanical constraints that develop within it in the 
(a)

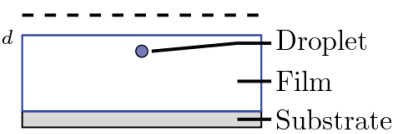

(b)

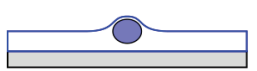

(c)
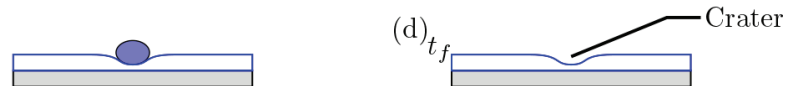

Fig. 14. Schematic representation of the steps leading to the crater formation. (a) Formation of drops at $t_{d}$, in the immediate neighborhood of the free surface; the dashed line indicates the initial level of the free surface; (b) growth of the drop by coalescence; (c) resurgence and destruction of the drop on the film surface; (d) formation of a persistent crater in the dry film. (Figure extracted from [55].)

last moments of drying. The schematic representation of fig. 14 summarizes the successive stages that lead to the formation of craters in the dry film.

The use of $\mathrm{X}_{\mathrm{W}}$ agents that are partially miscible in water but have a surface tension a factor of about two lower than phenoxyethanol, phenoxyisopropanol and phenol does not lead to any structuring of the film despite the demixing. Thus, it takes a particular combination of physico-chemical properties of the $\mathrm{X}_{\mathrm{W}}$ agent to produce the formation of permanent craters in the dry film.

\subsection{Conclusions}

The demixing caused by the evaporation of water is an interesting way of structuring aqueous thin films used in cosmetology. Depending on the nature of the constituents of the initial aqueous suspension, it is possible to form craters of relatively homogeneous size that persist in the dry film, without noticeably changing its final elastic properties. This structuring phenomenon could be put to use to design cosmetic films of a given porosity, making it possible, for example, to control the exchanges between the skin and its environment. The model presented in this work can serve as a starting point for many investigations not only in cosmetology but also in pharmacology, where use of microporous therapeutic films could have certain benefits in the treatment of certain pathologies, such as wounds or burns.

\section{Medicine and forensics applications}

From fluid mechanics point of view, blood is a complex colloidal suspension that behaves like a non-Newtonian fluid. Human blood is composed of different cells that form about $45 \%$ of whole blood volume and $25 \%$ of the total blood mass. They are classified as red blood cells (RBCs), or erythrocytes; white blood cells (WBCs), or leukocytes; and platelets (thrombocytes). The other $55 \%$ is blood plasma, which is composed of water (over 90\%), ions, electrolytes (salts), plasma proteins (7\%, most of which are by weight), and other substances transported by blood. The main physical properties of whole human blood, such as the density, viscosity, and surface tension, can be found in the literature [62].

Hereafter, we focus on the type of blood drying in droplet or pool geometry. Blood droplets and pools of blood are of huge interest in forensic science to retrieve relevant information from a crime scene. The scientific objective has succeeded in performing an accurate reverse engineering. The question to be asked is simple: from a fluid already spread on a substrate and already drying or dried, is it possible to obtain accurate and relevant information? But blood droplets spreading and drying are also of the tremendous potential of biomedical applications. Here also, a reverse engineering work could potentially reveal the blood composition based on the patterns observed in the dried droplet. Here the blood composition is the main complexity to perform a simple reverse engineering. Later we will present a few parameters that affect the final pattern of dried droplets.

\subsection{Drop of blood}

Evaporation of sessile drops of biological fluid is studied as a potential area of interest for medical applications. Most studies have been conducted on biological fluids and blood serum, but a few have focused on whole human blood. Several parameters influence the evaporation, gelation, cracking, and delamination processes of a drop of whole human blood such, as for example, the surface roughness or the ambient humidity rate. The first parameter influences the triple line stability when moving for example during the spreading. The second clearly changes the evaporation rate and then the stresses encountered in the colloidal suspension under phase change.

\subsubsection{Medical application}

One of the parameters that influence the drying rate, and thus the final pattern, is the ambient humidity rate, $R H$. The influence of the relative humidity on the pattern at the end of the drying phase has been investigated by considering the same volume of drops of blood evaporating in different $R H$ levels. Final drop images are presented in fig. 15, which shows morphological and structural evolutions of a drying drop of whole blood for different values of $R H$. For each $R H$, the bottom images are taken at the final stage of the drying process. One clearly observes a structural change and, consequently, the morphological evolution of the final drying pattern. At the final stage of the evaporation, the pattern is characterized by three distinguished regions: a central part composed of a sticking deposit and a network of small cracks, a corona composed of mobile plaques organized radially, and a fine periphery strongly adhering to the substrate. It can be observed that the width of mobile plaques in the corona and the fine periphery region become larger as the $R H$ increases. Also, fig. 15 shows that mobile plaques depend on the drying rate. At low drying rates (high $R H$ ), more 


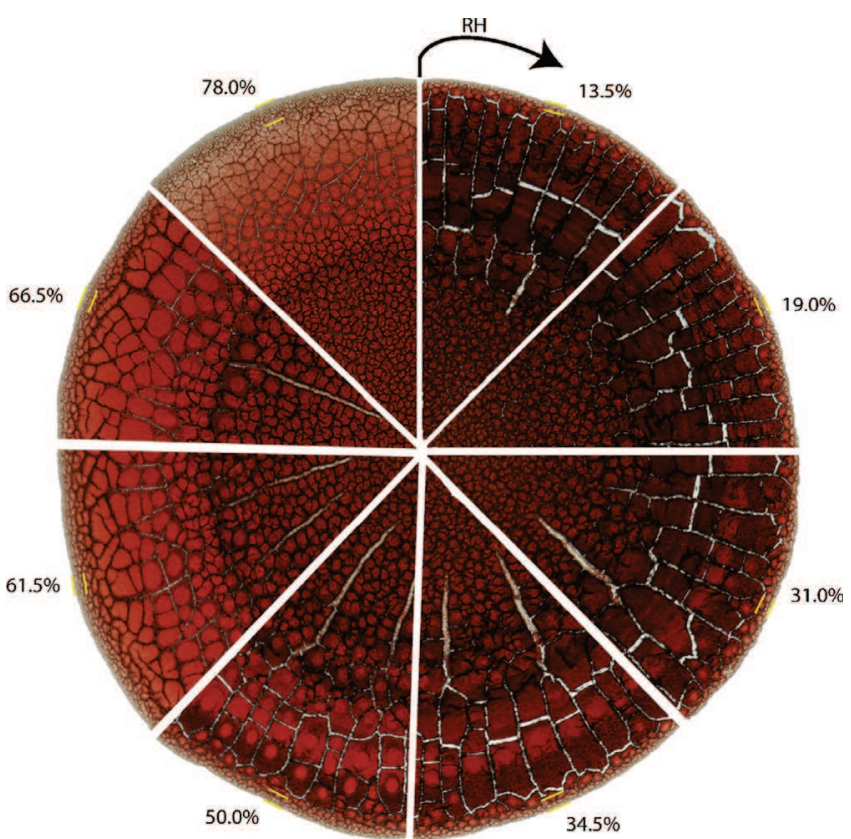

Fig. 15. Final deposition morphology for various $R H$ values ( $R H$ varying between $13.5 \%$ and $78.0 \%$ ). Area between dashes in yellow represents the fine periphery corona. Figure reproduced with permission from [63].

completely adhering mobile plaques are observed than at high drying rates (lower $R H$ ). Indeed, low adhering areas are highlighted by the circulated red light region that is found in each of the mobile plaques in the corona area. The final drying pattern and crack nucleation vary with the evaporation rate as presented in fig. 15. Under our experimental conditions, the transfer of water in the air is limited by diffusion and is controlled by the $R H$ in the surrounding air. The drying process of a sessile drop of blood is characterized by an evolution of the solution into a gel saturated with solvent. When the gel is formed, the new porous matrix formed by the aggregation of particles continues to dry through evaporation of the solvent that causes the gel to consolidate. As the liquid progressively recedes into the porous medium, it forms at first menisci at the air/solvent interface due to capillary tension between the particles and then causes liquid bridges between the particles. During solvent evaporation, the curvature of the solvent air menisci is responsible for a capillary pressure in the liquid phase. This depression induces shrinkage of the porous matrix that is constrained by the adhesion of the deposit to the glass substrate and the evaporation of the solvent. As tensile stresses build up, the internal stresses become too great, and fractures appear so as to release mechanical energy. Assuming that gelation is due to particles accumulation, we attribute these differences in pattern formation to the competition between the drying process and the adhesion of the gel on the substrate.

Final dried images presented in fig. 15 show that the final adhering area of mobile plaques is strongly dependent on the $R H$ values. By changing the drying rate, the mechanical properties of the drying gel are modified, such as the adhesion energy of the gel to the glass substrate (according to the Griffith theory). Indeed, the surface area of mobile plaques of drops of blood dried at $\mathrm{RH}$ from $13.5 \%$ to $50.0 \%$ becomes progressively larger with a lower adhering region. This adhering region shrinks until the formation of a circular adhering region that leads to a delamination process. For images of $R H$ above $50 \%$, mobile plaques are smaller with a higher adhering region. This observation is due to the buckling process that is rapidly overcome by the adhesion of the gel to the substrate. In desiccated colloidal gel dried at $R H=70 \%$ and $R H=46 \%$, Pauchard experimentally showed that complete adhesion or de-adhesion is a function of the cell surface area [64]. Moreover, the large fine periphery of the stick deposit onto the glass substrate is due to the receding of the gel front, causing the absence of RBCs. This result is due to a change in the internal flow that transports RBCs from the centre to the periphery of the drop of blood.

\subsubsection{Forensic application}

The first obvious parameter which influences the pattern of a dried drop of blood after impact is the impact velocity.

The added kinetic energy for the same volume leads to a larger spreading of the droplet and to a decrease of its thickness. The evaporation is thus preferred at the contact line, which generates a surface tension gradient that drives a stronger re-circulation, namely Marangoni flow, and for the RBCs to homogenize. Moreover, the coffee ring effect is exposed to a timescale competition between the water evaporation and the movement of RBCs. A clear coffee ring effect cannot be successfully observed if the water evaporates faster than the RBCs movement towards the periphery of the droplet. Hence in the case of a droplet impacting at a higher velocity for the same volume, the thinning of the drip stain thickness induces a faster evaporation and a change in the internal flows. The 3D profile visualisation of the dried bloodstain was used to characterize the features marking the rim dimensions in a radial cross section. Thus we were able to record the internal diameter of each droplet as shown in fig. 16, by averaging its measurement in different radial cross-sections, and
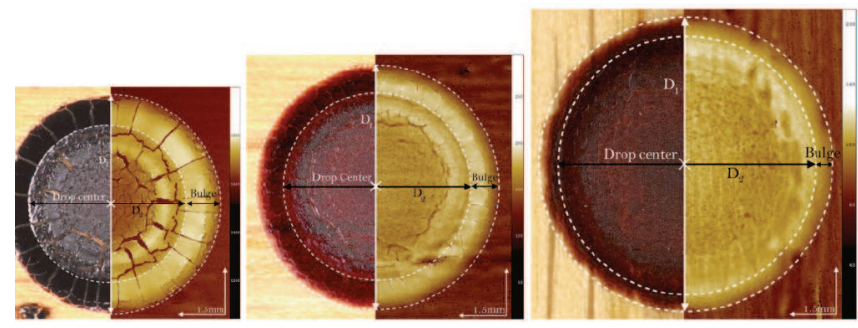

Fig. 16. View of dried blood drip stains with the bulging outer rim, from a healthy volunteer. Droplets of initial diameters of $2.4 \mathrm{~mm}$, and of impact velocities from left to right of $0.74 \mathrm{~m} / \mathrm{s}$, $1.87 \mathrm{~m} / \mathrm{s}$ and $4.75 \mathrm{~m} / \mathrm{s}$. On the left side with photography, on the right side with confocal microscopy that gives the $3 \mathrm{D}$ features by means of radial cross-sections. Figure reproduced with permission from [65]. 


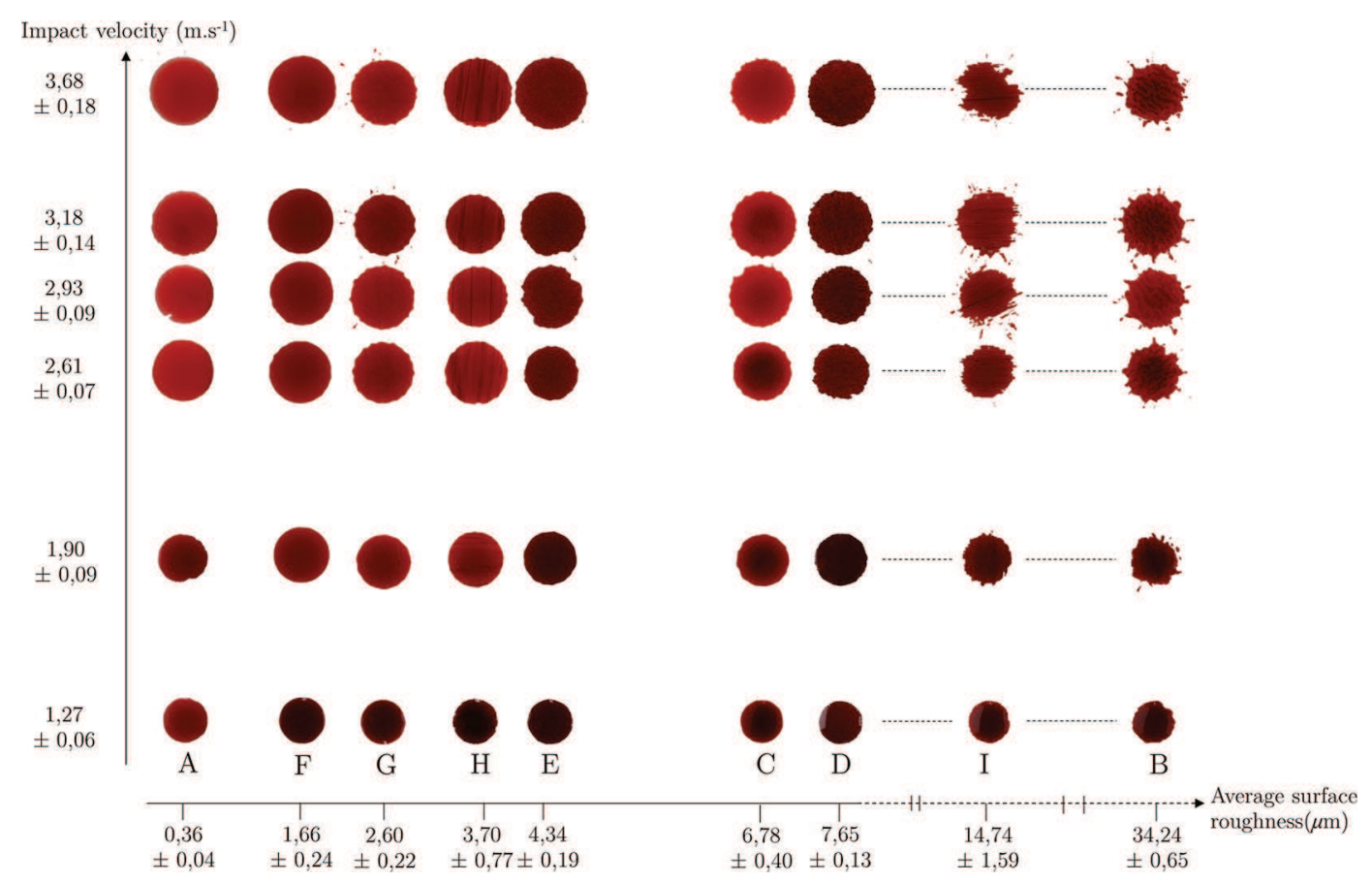

Fig. 17. Increasing average surface roughness influence on the pattern for same impact velocity of $2.6 \mathrm{~m} / \mathrm{s}$. Figure reproduced with permission from [66].

comparing it to photographic analysis. Another parameter to be accounted for in the frame of forensic analysis is the roughness that influences the outcome of the final patterns after impact. With the high-speed recordings, we were able to classify our impacting droplets as either splashing or non-splashing. But what can be observed with the high-velocity camera is not always visible after impact by photographing only the drip stain features. Thus, with the pictures taken right after the impact of the blood drip stains, we create the visual representation given in fig. 17, which shows the appearance of the stains, from a top view, ordered according to their impact velocities and the roughness properties of surfaces. The roughness has already been shown to be a key parameter playing a role in the behaviour of impacting blood droplets. From left to right, the arithmetic average of the surface roughness increases, thereby indicating smooth surfaces on the left and rough surfaces on the right of the figure. As can be seen, drop impacts on the smooth surfaces result in stains with smooth edges, no deformation, and only some satellite stains at higher impact velocities. The two roughest surfaces, B and I, show clearly deformed edges, where a nice circular shape cannot be identified: large irregularities are visible on the edges of the parent stain. In between the smooth and rough substrates, surfaces $\mathrm{G}, \mathrm{H}$, E, C, and D show some small deformations around the circular edge, in the form of scallops, although the circular structure remains clearly visible. As expected, rough surfaces present clearly visible satellite stains, but surface $\mathrm{G}$ appears to have its satellite stains clearly visible as well, unlike the other surfaces of similar roughnesses. However, this means that only the satellite stains on this surface are of a larger volume than for the other surfaces of similar roughness and thus become visible with a topview picture. For the other surfaces, the satellite stains are too small. Moreover, the edge of the parent stain is not really deformed. Hence, the surface roughness not only influences the splashing limit but also is of major influence in the smoothness of the edge of the parent stain. Additionally, the irregularity of the parent stain seems to increase with the impact velocity: the higher the impact velocity, the more irregular this edge will be. This, as well as the increasing diameter, is in line with the observations in earlier publications $[67,68]$. One assumption to explain these observations is that the rougher the surface is, the more difficult it will be for the entrapped air to escape. On very smooth surfaces, the skating on a thin air layer would favour fingering-link splashing, whereas, on a rough surface, more stress would act on blood, changing its viscosity, and the splashing would resemble more a corona-like splashing. Surface G would correspond to this transition, where large fingerlike droplets are observed. Data and observations for the treated surfaces have not been incorporated into this figure. However, photographs and high-speed recordings have been compared one to one with the drop impacts of the untreated surfaces. We find that wettability does not significantly influence the splashing limit or the appearance of the drip stains presented in fig. 17. On the other hand, the pattern of the dried stains exhibits some small differences between the non-treated and treated surfaces, depending on the surface. During the drying of the drip stains, two different evaporation regimes take place [48], where the colloids present in blood are redistributed and the stress induced by the evaporation of water, the main component of plasma, leads to noteworthy cracks. For surfaces $\mathrm{C}$ and $\mathrm{H}$, less cracking is observed 


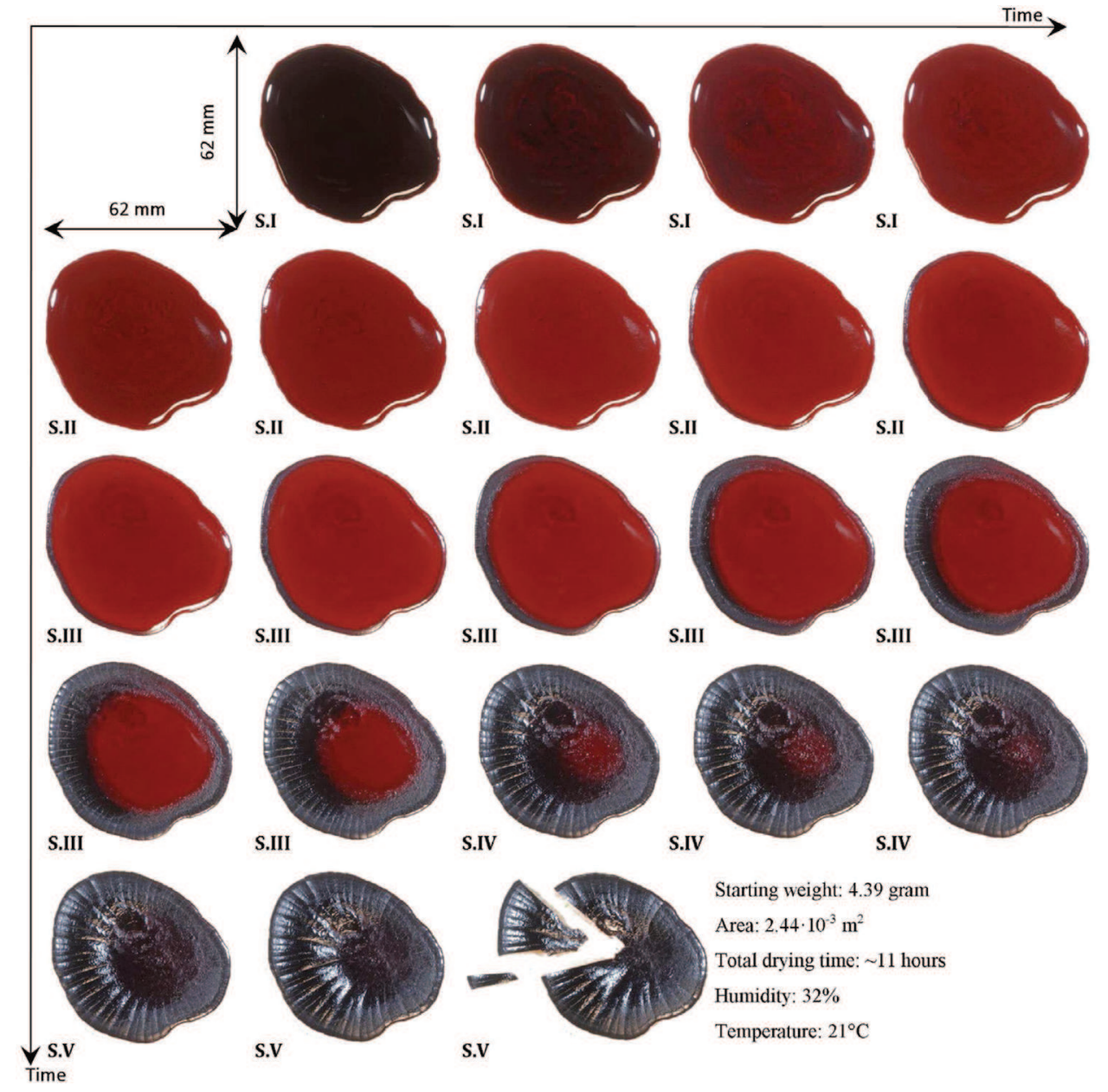

Fig. 18. Time-lapse of a drying pool of blood from a healthy person, at $21.8^{\circ} \mathrm{C}$ with a relative humidity of $32 \%$. Note that the elapsed time between pictures is different for each stage; the rows follow one another over time. Figure reproduced with permission from [69].

for low impact velocity stains when they impacted the treated (hydrophobic) surfaces compared to non-treated (hydrophilic) surfaces. It seems that the wettability of the surfaces plays a role in the drying process by inducing some stresses on the cracking features of a drying drip stain.

\subsection{Pool of blood}

In fig. 18 we show a time-lapse of a drying blood pool deposited on a linoleum surface. First of all the simple observation of the pictures obtained in the experimental conditions described previously allowed the identification of five distinct phases: (I) coagulation stage, (II) gelation stage, (III) rim desiccation stage, (IV) centre desiccation stage, (V) final desiccation stage. These stages are detailed below:

- (I) When blood is deposited upon a surface, the RBCs are evenly distributed throughout the pool, which then will sediment. At this point the blood has a dark red colour and starts to coagulate [70]. It is possible that due to wetting and capillary action, the area of the blood pool increases as the blood spreads slowly over the surface during the initial 15-30 min. During this first stage there is a change in colour from dark red to lighter red, mainly due to coagulation.

- (II) As fluid evaporates from the blood pool red blood cells, that are not constricted in the fibrin web, are transported to the rim of the stain and deposited, due to flow caused by evaporation [48]. The transition from the fluid to the gel state is referred to as the gelation front. The second stage starts when the gelation rim is created around the pool. The gelation front propagates inwards, towards the centre of the stain, as the pool continues to dry.

- (III) The third stage starts as soon as the rim turns black and starts to crack, indicating that the rim is desiccating. The transition from the red to black colour is referred to as the drying front. During this stage both the gelation and drying front propagate towards the centre of the stain.

- (IV) Once the gelation front reaches the centre of the stain, the entire stain has gelled and evaporation of 


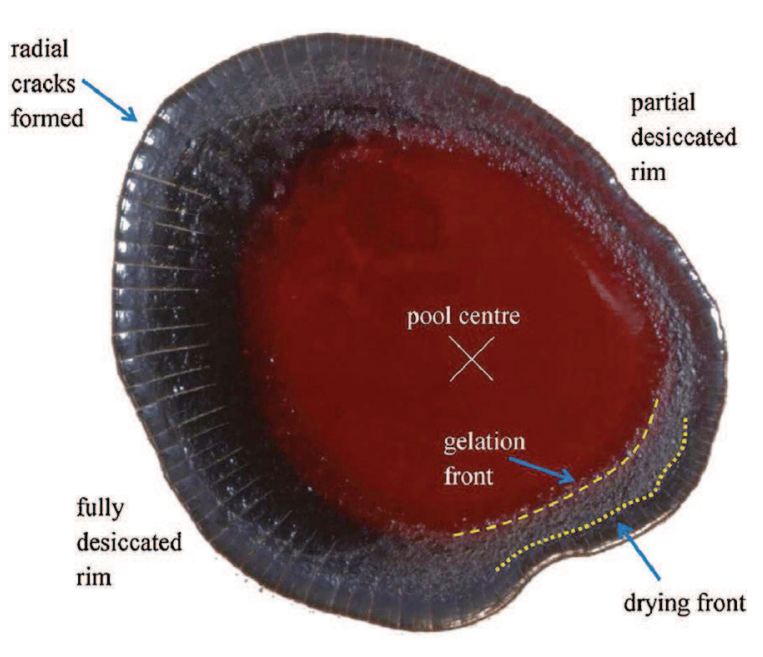

Fig. 19. Close-up view of a drying pool, with several defined properties of the pool. The yellow dashed and dotted lines represent the gelation and drying front, respectively. Figure reproduced with permission from [69].

fluid is mainly driven by the porous media drying dynamics [71]. The drying front and cracks propagate towards the middle of the stain.

- (V) Finally, the drying front reaches the centre of the pool. The pool has almost completely desiccated. During this last stage, the entire pool is black in colour. As the last liquid evaporates, the remains contract and the cracks reach the middle of the stain. Accordingly, flakes are separated and partially or completely detach from the surface.

We have observed the five stages described above for every pool we created. However, it should be clear that a pool does not dry in a uniform manner. Instead, one part of the blood pool may be fully desiccating (left side fig. 19) while another part is still in a gel-like state (right side fig. 19). Consequently, the centre of the pool, i.e., the location where all cracks come together, does not necessarily has to be the geometrical centre of the pool. Furthermore, the duration of any one stage and complete desiccation can differ between blood pools, depending on the humidity, temperature, shape and size of the pool and the kind of surface.

Literature evidence that drying dynamics of pools of whole blood is investigated for a small range of volumes (3.5-4.5 mL). As stated by Laan et al. [69], it's possible to distinguish five different drying stages, each with their own characteristics. The mass of a blood pool diminishes in a very reproducible manner, first linearly in time and then approaches a constant value. The general knowledge concerning blood pools within the A step forward in the classification and characterization of blood pools is still needed. Prospectively, these results could be used for crime scene reconstruction or for future investigations into determining the time the blood shedding event occurred.

\section{Drying colloidal layers: a way to investigate the pictorial matter}

Crack patterns are one of the most apparent signs of ageing of an easel painting. Most ancient paintings display craquelures that affect more or less their visual aspect (fig. 20).

The multitude and diversity of crack patterns are related to a large number of processes occurring all over the lifetime of a painting. A crack in a paint layer can occur due to primarily chemical and physical action as a consequence of a too fast drying process, or later when the painting is subjected to a loss of mechanical strength, or environmental changes such as oxidation, the effect of UV or cycles of relative humidity and temperature, ....

The support expansion also results in cracks and possibly in a loss of adhesion whatever the support (canvas, wood panels, ...). As well, vibrations or shocks due to transports lead to particular crack patterns. Some specific crack patterns can reveal elements of the lifetime of a painting. More generally, the study of crack pattern in artworks is guided by several motivations. Crack patterns possibly reveal some properties of the pictorial matter or some information about the methods used by the artists which in turn can provide important details to authenticate paintings [2]. For example, the crack pattern can reveal anisotropy in the pictorial matter, the brush marks or qualitative information about the mechanical properties of the pigments used, or the layer thicknesses. Studies of crack patterns are of great interest in the domain of conservation and restoration of cultural heritage. Such studies focus for example in the stability of a crack pattern, that is how a crack pattern can evolve in relation to the surroundings or with the potential curator interventions. For these reasons, cracks patterns are studied in modelled systems in order to isolate the effect of a particular parameter on the morphology of the paint layer.

\subsection{Pictorial layer vs. model system}

Generally, the large variety of crack patterns in paintings follows from the complexity of the paint layers both in terms of geometry and physico-chemical properties. Most of the paintings are composed of layers based on a deformable support, canvas or panel (fig. 21(a)). A paint layer consists of a film-forming component, e.g. the binder (oil or water miscible in the case of tempera method), and organic or inorganic colourants, e.g. the pigment usually from 1 to several tens micrometres size. Different kinds of additives such as surfactant, plasticizer, or solvent can be mixed in the paint to improve its viscosity needed for paint deposition and its stability. The composition of these materials has strongly varied through history. Indeed, the pigments, binder and additives strongly affect the structuration as well as its ageing of the paint layer. Moreover, the evolution of the rheological and mechanical properties of the paint layer strongly evolve over the time. Just after its application, the behaviour of a paint layer changes from 


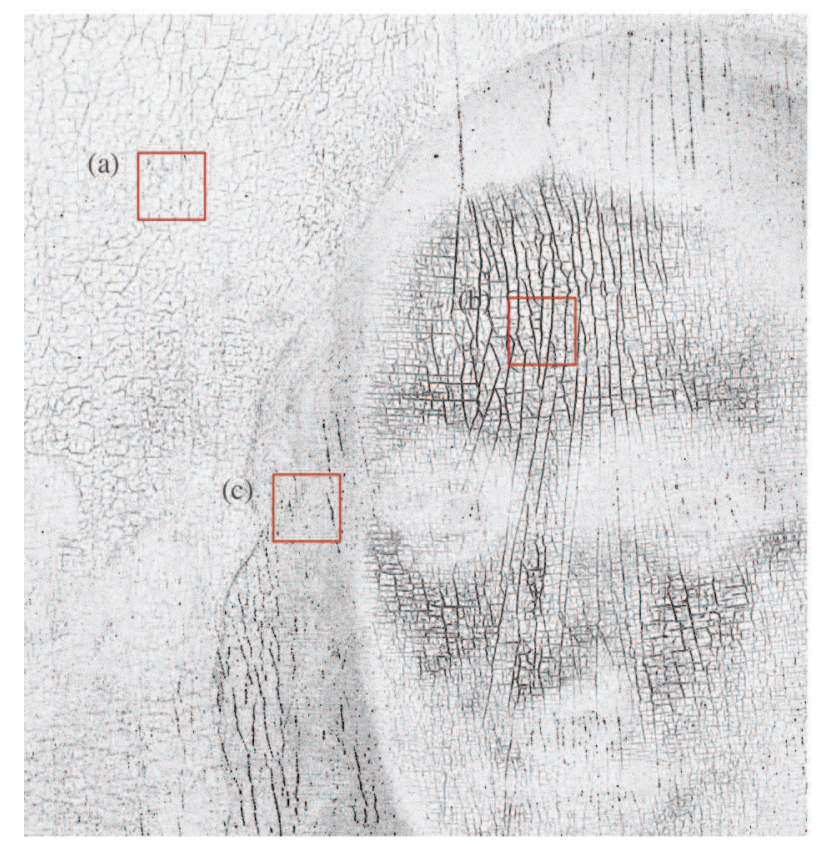

(a)

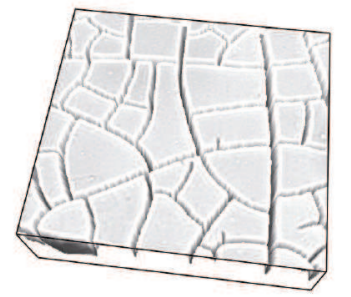

(b)

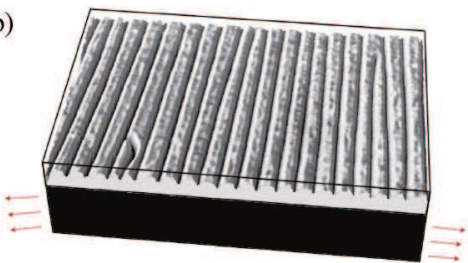

(c)

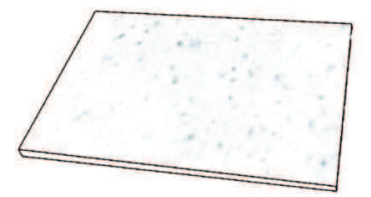

Fig. 20. Various crack patterns in some parts of Mona Lisa (reproduced from [2], with the permission of AIP Publishing), and similar patterns in modelled systems. (a) Hierarchical formation of drying cracks in a drying colloidal layer (100 $\mu \mathrm{m}$ thick), associated with isotropic ageing cracks in the sky of Mona Lisa. (b) Array of parallel cracks in a brittle colloidal layer (20 $\mu \mathrm{m}$ thick) on a stretched sublayer, associated with deep cracks in the forehead of Mona Lisa. (c) Crack-free layer ( $2 \mu \mathrm{m}$ thick), and associated thin layer using the Sfumato techniques in Mona Lisa. Reproduced from [2], with the permission of AIP Publishing.
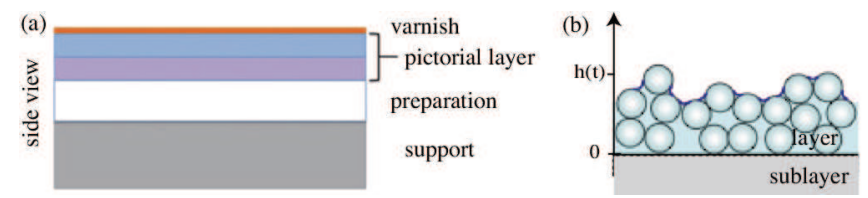

Fig. 21. Sketch of the structure of a paint layer (a) and model system (b) in side view. In colloidal model systems, the cohesion of the particulate layer, also the adhesion on a sublayer are tunable.

a classical Newtonian behaviour to a visco-elasto-plastic behaviour, then to a brittle behaviour. These changes in the rheological behaviour are attractive to painters to paint faster or to tune the visible aspect of the pictorial matter such as freezing the brushmarks as a result of the levelling effect (see inset in fig. 22): the leveling properties of paint materials were applied to van Gogh's paint layers [72]. In order to confirm or invalidate the underlying assumptions on the origin of a specific crack pattern, modelled experiments are investigated. This approach is based on the fact that a specific cracks pattern depends on both the mechanical properties of the matter and the loading mode. Thus, working with model systems provide the possibility of reproducing specific crack patterns as a function of the mechanical properties of the system, and key parameters such as the layer thickness, the adhesion on a sublayer, or the drying conditions, .... However, even if modelled systems can be adapted to develop a specific crack pattern, these modelled experiments do not pretend to mimic the overall processes that occur during the life-

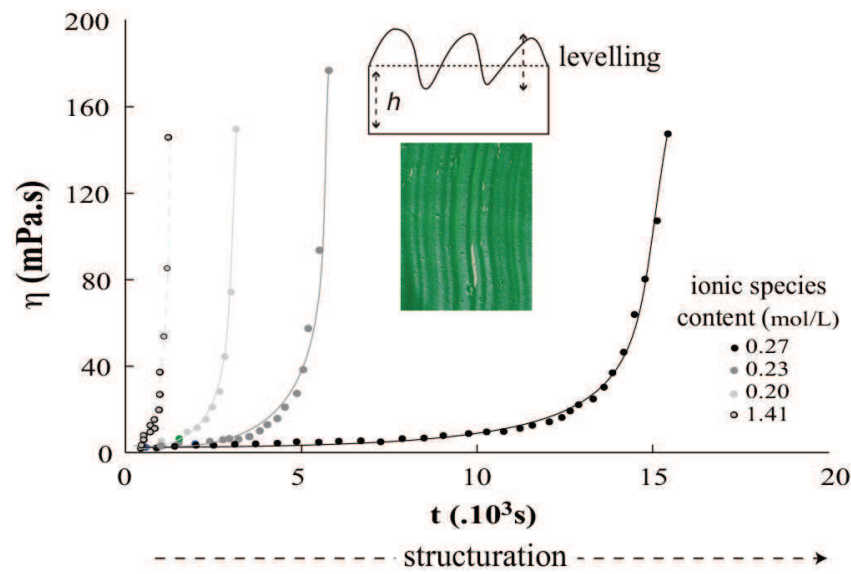

Fig. 22. Viscosity of silica dispersions as a function of time for different ionic species contents, in $\mathrm{mol} / \mathrm{L}$, in the absence of evaporation (measurements were done with an imposed shear rate rheometer). Inset: cross section showing paint layer irregularities related to brush marks and top view presenting the brush marks in a real paint layer. Reproduced from [73]. Copyright (c) 2018 Elsevier Masson SAS. All rights reserved.

time of a painting, that is still not well known, but the use of analog experiments aims to isolate specific parameters affecting the solidification and the resulting crack pattern.

Modelled systems are layers of concentrated aqueous dispersions of nanoparticles on a non-porous substrate (fig. 21(b)). Nanoparticles are either pure stiff silica particles or binary mixtures of stiff and soft polystyrene par-

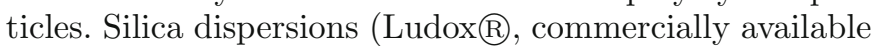


from Sigma-Aldrich) are characterized by different particle sizes, often in the range $10-25 \mathrm{~nm}$, and different species of counter ions. Note that the tiny particles used as a model do not correspond to the pigment size that ranges, on average, between $1 \mu \mathrm{m}$ to more than tens of $\mu \mathrm{m}$ depending on the pigment mechanical properties and the way they were ground. However, the nanoparticle size is close to the lengthscale that is associated with the mean roughness of the real pigments [74]. In particular polystyrene particles exhibit a specific glass transition temperature, that is estimated from the particle core composition; this property governs the mechanical properties of the particles. The choice of the colloidal system provides a medium tunable and well-defined rheological and mechanical properties [4]. During the solvent evaporation, that is limited by diffusion of water in the air, the density of particles increases until the formation of a close-packed network of particles whose pores are still filled with solvent, e.g. gel phase. Rheological characterization reveals that colloidal dispersions vary from viscous to elasto-visco-plastic to brittle when the water content decreases. This transition toward a gel phase can also be investigated in the case of charged particles without solvent removal but by tuning the interparticle forces through the addition of ionic species to the dispersion [75]. This solidification process is accompanied by complex changes in the rheological behaviour of the dispersions [4]. After a slow increase, the viscosity, $\eta$, exhibits a sharp increase and finally diverges (fig. 22). The time evolution of the viscosity is well captured using a percolation model as:

$$
\eta \sim \eta_{s} /\left(t-t_{g}\right)^{s}
$$

where $\eta_{s}$ is the viscosity of the solvent [76]. The duration elapsed between the sample preparation and the gelation transition, e.g., the gelation time $t_{g}$, strongly depends on the ionic species concentration. It finally results in the generation of a structure exhibiting a finite shear modulus.

The main physical parameters of the gel phase are the elastic modulus, the strength and the yield stress that strongly depend on the pigment size, pigment stiffness, the inter-pigment force and the pigments concentration. In particular, these quantities can be measured macroscopically through different techniques, in both paint layers and modelled systems: tensile-loaded conditions or indentation testing [2,77] (fig. 23). The formation of cracks can then be generated by the effect of the substrate expansion or due to the drying process. In the last case, due to the action of the capillary forces at the surface of the gel phase, solvent evaporation induced a transport of liquid from the porous structure. The local liquid pressure, $p$, in the pores obeys a one-dimensional diffusion equation along the $z$ vertical direction:

$$
\frac{\partial p}{\partial t}=\frac{k_{p} E}{\eta} \frac{\partial^{2} p}{\partial z^{2}}
$$

where $E$ is the elastic modulus of the gel phase, assumed to be time-independent, and $\eta$ is the viscosity of the solvent flowing through the porous structure of permeability $k_{p}$.

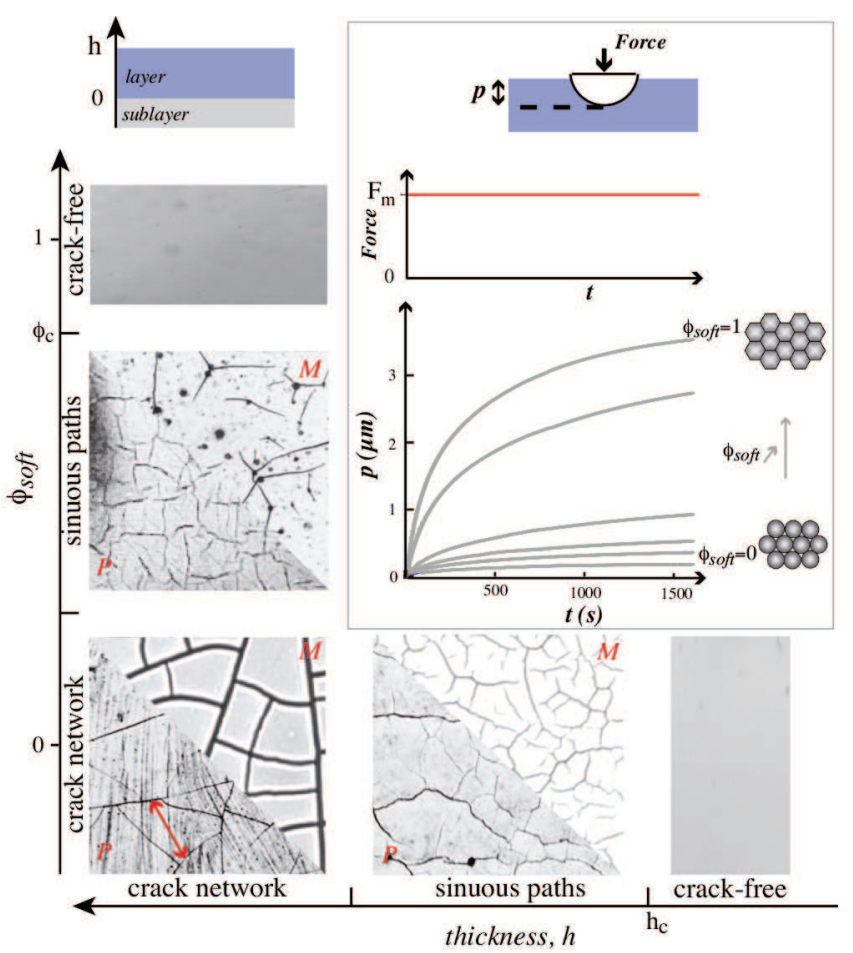

Fig. 23. Crack patterns diagram in different planar layers as a function of the layer thickness (horizontal axis), and composition (vertical axis). Images of real paint layers are indicated by " $P$ ", and modelled colloidal layers are indicated by " $M$ ". modelled colloidal layers are formed by binary mixtures of both stiff (solid volume fraction $1-\phi_{\text {soft }}$ ) and viscoplastic $\left(\phi_{\text {soft }}\right)$ particles. Mechanical characterization of modelled systems uses indentation testing: creep comparison of different colloidal gels was investigated by driving a spherical tip (diameter $200 \mu \mathrm{m}$ ) inside the sample; then the time variation of the penetration depth, $p$, was measured at constant load, $F_{m}$.

Hence, eq. (19) results in a pressure distribution in the liquid pores. At first approximation, the drying stress, $\sigma_{i, j}$, averaged over the layer thickness, linearly increases with time as:

$$
\overline{\sigma_{i, j}}(t)=E t / t_{D},
$$

where $t_{D}$ the evaporation timescale [78]. The occurrence of cracking can be more or less delayed as a result of a mismatch between the solid strength and the stress buildup. This mismatch will govern the way the layer is invaded by cracks: cracks density, connexion orientation, ....

Both following sections are devoted to give examples of modelled experiments intended to reveal some properties of the paint layers.

\subsection{Crack patterns}

Since cracks formation is a way to optimize stress release all over the system, their patterns reveal some mechanical properties of the matter. Under fixed drying conditions, morphologies of cracks depend on both (I) the layer thickness and (II) the composition modelled by a binary mixture of stiff and deformable particles (diagram in fig. 23). 
- (I) For a linear elastic material, the propagation of a crack involves the energy available for crack growth, e.g. the energy release rate, $G_{c}$, and the work, $W$, used to create the new crack surfaces in the layer. Thus propagation of a crack is governed by the energy balance: $G_{c}=W$, that applies to brittle materials.

A key parameter in the formation of a network of cracks is the layer thickness. Indeed, this quantity controls the energy that is possibly released through mechanical instabilities. The influence of the layer thickness on the crack pattern is depicted along the horizontal axis in fig. 23. Hence, below a critical thickness, $h_{c} \sim 1 \mu \mathrm{m}$, that depends on the elastic properties of the system, layers are uniform and free of cracks. Typical thin layers can be obtained by applying dilute paint layers. An example of crack-free layers depicting the veil of Mona Lisa is shown in fig. 20(c). Such thin layers were obtained through the famous Sfumato techniques probably consisting in a layer-by-layer deposition of dilute paint layers.

For increasing layer thicknesses, cracks propagate along sinuous paths that result in a partially connected network. For still thicker thickness, the hierarchical formation of cracks divides the plane into polygonal adjacent fragments [79]. The mean fragments size then increases with the layer thickness and depends on the stiffness of the layer.

- (II) However, a part of the energy of the system is dissipated more or less significantly through plastic losses. The plastic losses modifies the energy balance by consuming a part of the energy, $U_{p}$, as: $G_{c}=W+U_{p}$ [80]. A way to introduce plastic losses in modelled experiments is to tune the composition by varying the volume fraction of both stiff particles, $\phi_{\text {stiff }}$, and viscoplastic particles, $\phi_{\text {soft }}$, taking into account the condition $\phi_{\text {soft }}+\phi_{\text {stiff }}=1$. Hence, similar crack patterns to those indicated in (I) can be observed for constant layer thickness but different layer compositions (vertical axis in fig. 23). In particular, for layers with $\phi$ higher than 0.5 , indentation responses reveals a strong creep behaviour of the medium and crack-free layers.

The topology of the networks depicted in fig. 23 are recoverable changing the components of the layers. Indeed, a paint layer is usually made of pigments in a mixture of non-volatile and volatile solvents. The presence of a non-volatile solvent modifies the kinetics of solidification; consequently the transitions between crack-free, partially connected and fully divided layers are modified.

Moreover, throughout the life of a painting, the physical properties of the paint layer evolve from the solidification to the ageing. Young paint layers generally exhibit a ductile behaviour where the plastic work of fracture is significant. On the contrary, ageing cracks form later in the life of the paint film and result from brittle behaviour.

\subsection{Effect of the multi-layered structure}

The crack pattern in a layer strongly depends on the mechanical properties of the sublayer. The layered structure
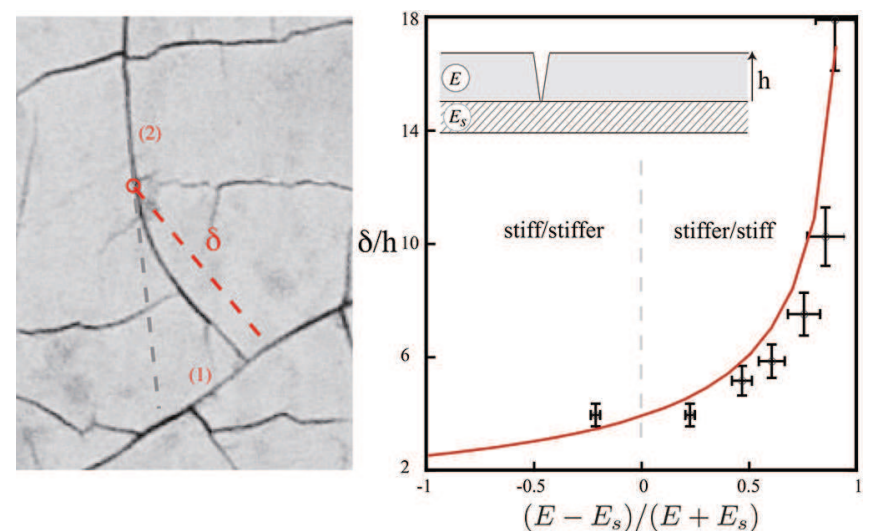

Fig. 24. Interaction between cracks. The path of crack (2) is modified by the stress release induced by the presence of crack (1). The lengthscale, $\delta$, related to the crack interaction is a function of both the layer thickness and the mismatch between the layer and the sublayer stiffness. Dots are measurements in modelled crack patterns and dark line is the theoretical prediction [81]. Reproduced from [2], with the permission of AIP Publishing.

usually consists of a stiff layer on a less stiff one but the opposite case can also be encountered. In this way, a key parameter is a ratio between the elastic moduli of the layer and the sublayer, $E / E_{s}$ (fig. 24). One crack defines the boundary conditions for the mechanical stress field that will govern the formation of the future cracks. Therefore, at some distance from a pre-existing crack path, crack (1) in fig. 24, the stress remains unrelaxed on a lengthscale due to the constraint of the substrate. A second crack, crack (2), will change its path when it approaches the first one at a distance less than $\delta$. This lengthscale can be easily measured on a pattern of cracks leading to estimate the ratio $E / E_{s}$. The ratio between $\delta$ and the layer thickness, $\delta / h$, is plotted for different layered structures in fig. 24. The lengthscale $\delta$ is found for small strain that is usually the case for paint layers, to be equal [81] to

$$
\delta / h=\frac{\pi}{2} g(\alpha, \beta)
$$

where $g$ is a numerical function which depends on the Dundurs parameters $\alpha(-1<\alpha<1)$ and $\beta(0<\beta<$ $\alpha / 4)$ functions of the plane-strain elastic moduli and on the Poisson ratio of both the film and the sublayer $(\beta$ typically has only little influence on the results compared to the influence of $\alpha$ ). In the limit of a very soft sublayer, $\delta$ diverges and the film behaves as if it is not constrained.

\section{Drying as an analog model to study crack patterns in nature}

What does drying starch teach about the graceful columns formed in cooling lava? How does watching mud dry and crack inform us about the evolution of permafrost on Mars? In this section these questions will be answered, and the use of drying systems as analogue models of different fracture patterns in nature explored. 


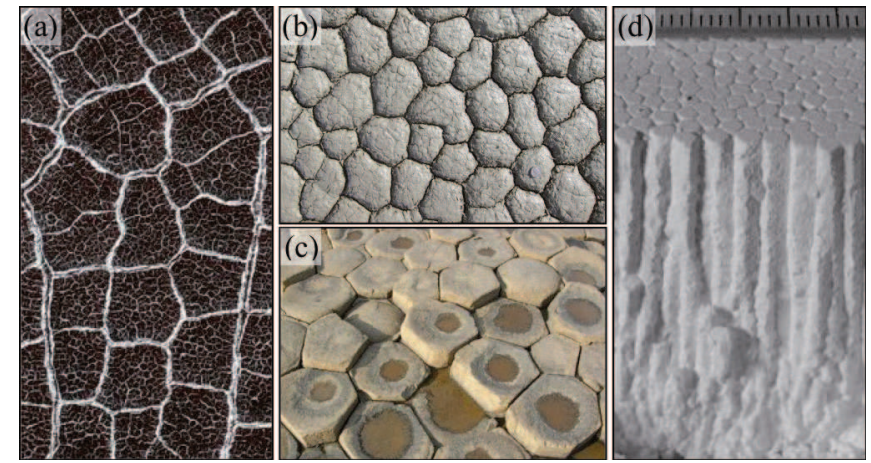

Fig. 25. Examples of crack patterns in nature, showing analogies between cooling and drying. (a) Polygonal terrain on Mars (image credit: NASA/JPL-Caltech/University of Arizona). (b) The evolution of crack patterns in dried clay can simulate the dynamics of polygonal terrain $[82,83]$. (c) Columnar jointing of the Giant's Causeway demonstrate a pattern that is commonly found in cooled lava (image courtesy of S. Morris). (d) Columnar jointing also forms in dried starch, although columns are typically only a few millimeters in diameter [84-86].

\subsection{Cooling vs. drying}

There is an exact mathematical analogy between the physics of how a porous solid dries, and how a hot solid cools. Thus, simple experiments in one domain, such as drying clays [83] or starch [84], can be used to provide insight into more challenging questions in the other domain, such as how permafrost responds to seasonal temperature variations [82], or how lava breaks as it cools [87]. Some examples of such related patterns are shown in fig. 25 .

The full analogy can be developed in detail, as in refs. [88-91], and relies on the similarity of how heat or fluid affects a material body. Starting from an appropriate thermodynamic potential, a change in free energy density can be caused by the addition of either some heat, $T d s$ (for entropy per unit volume, $s$, at temperature $T$ ), or fluid, $p d \vartheta$ (for fluid content per unit volume, $\vartheta$, at pressure $p$ ). Following one of these contributions through, and coupling it to linear elasticity leads to the theories of linear thermoelasticity or poroelasticity, respectively. This physics underlies many other results, for example the pressure diffusion of eq. (19), derived in ref. [92]. It is also fully consistent with the balance equations presented for colloids in sect. 2.3, above, despite some differences in conventional notation.

For heat, the thermoelastic momentum and energy balances can be expressed as

$$
\nabla \cdot \boldsymbol{\sigma}=\alpha_{T} K \nabla T,
$$

and

$$
\kappa_{T} \nabla^{2} T=\rho c_{v} \frac{\partial T}{\partial t}+\alpha_{T} K T_{0} \frac{\partial \epsilon_{i i}}{\partial t} .
$$

Here, $\alpha_{T}$ is the thermal coefficient of expansion, $K$ the bulk modulus, $\kappa_{T}$ the thermal conductivity, $\rho$ the density at reference temperature $T_{0}$, and $c_{v}$ the specific heat at constant volume, for the material being modelled. Any thermal expansion or contraction can generate internal stress via eq. (22), where it contributes an effective body force to the conditions of stress equilibrium. The dynamic component of how heat moves is captured by eq. (23). The first two terms are more familiar as the heat diffusion equation. The last term describes how heat is generated by adiabatic compression, as the body is strained. It is, however, usually negligibly small when considering the thermodynamics of elastic deformations.

For fluids infiltrating a porous solid body, such as might be the case for a drying paint, coating or soil, the corresponding model of poroelasticity, originally developed by Biot $[88,92]$ can be written as

$$
\nabla \cdot \tilde{\boldsymbol{\sigma}}=\alpha_{p} \nabla p,
$$

and

$$
\frac{k_{p}}{\eta} \nabla^{2} p=\frac{1}{M_{\epsilon}} \frac{\partial p}{\partial t}+\alpha_{p} \frac{\partial \epsilon_{i i}}{\partial t} .
$$

Now one sees how the fluid pressure $p$ takes the place of temperature, when $\tilde{\boldsymbol{\sigma}}$ is the effective stress felt by the solid network that holds the body together. The pressure can be related to the curvature of any meniscii or capillary bridges in the wet porous body - although it can equally well be defined even in the absence of such interfaces [93]. The thermoelastic variables have also been transformed. In place of a thermal expansivity $\alpha_{p}$ is the Biot-Willis coefficient, which relates the compressibility of the porous body to its individual constituents [90,91]. In cases where, like drying paint or loose soil, the porous material is soft compared to its parts (e.g., hard colloidal particles or grains of soil), $\alpha_{p}=1$. A second poroelastic modulus is needed to complete the model: the specific storage modulus, $1 / M_{\epsilon}$, at constant strain. This characterises how much fluid can be pushed into or sucked out of the porous medium, if the fluid pressure is changed. Finally, $k_{p}$ and $\eta$ are the permeability of the porous media, and the viscosity of the fluid travelling in it, as in Darcy's law, from which they derive [92]. When strains can be neglected, eq. (25) reduces to a simple pressure diffusion equation of the form previously seen in eq. (19), analogous to thermal diffusion. Similarly, the fluid content of the body will also often behave diffusively [90-92].

One can now see that fluid with a pore pressure $p$, acting within a drying body, can induce stress in the same way that a change of temperature can. In other words, the internal pressures of the liquid can act as the driving force for contraction, and fracture. Generally, the thermodynamic stress $\sigma_{i j}=\tilde{\sigma}_{i j}-\alpha p \delta_{i j}$. This relationship, where $\tilde{\sigma}_{i j}$ is sometimes called the Terzaghi effective stress [94], allows the pore pressure to appear as a body force, like gravitational forces or internal forces generated by electromagnetic interactions.

In the context of drying, many applications of poroelasticity were developed by Scherer [95-97], and the interested reader is recommended to his original articles. More classic work includes the detailed review of poroelasticity by Rice and Cleary [98], and Wang's textbook on the subject [90]. Other relevant reviews and books have 
been published recently $[91,99,100]$, while an accessible popular-science review is available in ref. [101].

Interestingly, the connections from cooling and drying extend further, and similar physics also arises in the differential growth of tissue layers, such as leaves [102], or skin [103]. While there is no obvious "diffusing" quantity in these problems, differential growth can contribute to the development of stress or strain in living tissue in the same way as the effective body forces of heat or moisture. This suggests the possibility of applying analogue experiments in drying to targeted questions in biophysics.

\subsection{Columnar jointing: starch and lava}

As lava cools, it will often crack into regular prismatic columns. The seemingly unnatural beauty of landscapes of columnar basalt is evoked by the names of their most famous landmarks: the Devil's Postpile, Fingal's cave (fig. 26(a)) or the Giant's Causeway. Historically, this last site was the first to attract professional scientific attention, which appears in the form of travellers reports and letters published by the Royal Society in the 17th century [104-106]. By Victorian times the volcanic origin of basalt was well appreciated, and it was convincingly argued that the pillars grew along the thermal gradients of cooling lava, and were due to thermal stresses [107]. Around this time Huxley also described the columns of the Giant's Causeway as "not altogether unlike those into which a mass of starch splits during drying" [108].

The similarity of dried starch to columnar lava was rediscovered several times $[84,109,110]$. The work of Müller $[84,111,112]$, however, began the modern interest in analogue experiments with starch. Over the past few years, there has been a rigorous series of experiments involving potato starch $[85,113]$ and, more commonly, corn starch $[86,87,113-119]$. A typical experiment consists of mixing a few hundred grams of commercial starch powder with an equal mass of water, and some antiseptic such as a tablespoon of household bleach. If the resulting slurry is left to dry, it will crack into a colonnade, with columnar joints slowly extending down from the drying surface. Heat lamps are often used to accelerate this process. The result of breaking open such an experiment is shown in fig. 25(d). Potato starch is coarser than corn starch, and this variation in grain size can be linked to subtle differences in how these materials dry and crack $[113,116]$, but the basic experiment is similar in both cases.

Drying starch experiments have focussed on two questions: what sets the scale of columnar joints, and why are they so well ordered?

The columns in both starch and lava arise from contraction, as an initially homogeneous block of starch dries, or pool of lava cools. Water or heat is lost from exposed surfaces, which shrink as a result. Contraction cracks then open along the surfaces of, say, a lava lake like Kileui Iki, in Hawaii $[120,121]$. As time passes the lava continues to cool, from its edges inwards, and the cracks propagate deeper into the lava. Water may seep into the cracks and boil, maintaining an efficient heat transport through the
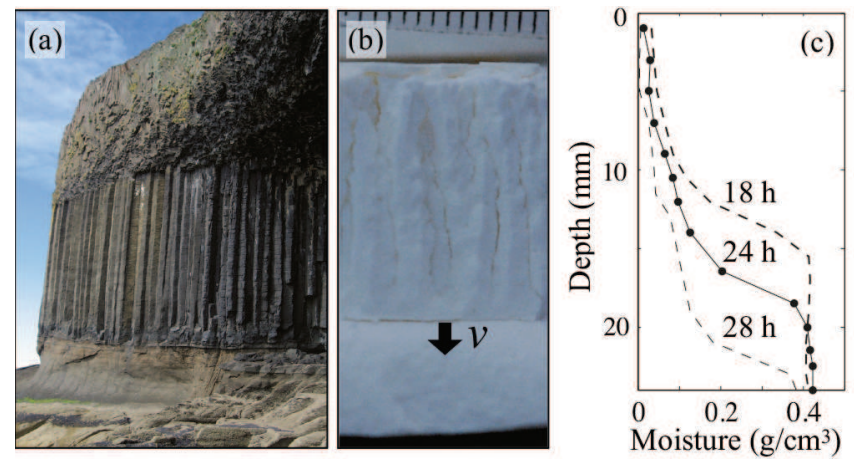

Fig. 26. Columns in lava and starch. (a) Near Fingal's Cave, two types of columns are visible in lava emplaced over a lightcoloured ash. The cracks that delimit the large regular columns of the lower colonnade invaded from the bottom of the flow, and grew upwards, tracking an isothermal surface, or cooling front. The upper layer consists of small disordered columns, or entablature. (b) In starch, drying can be interrupted: the columns here have been advancing into the drying starch, from the top down. The crack tips around the columns are all confined in a very thin layer, which advances into the drying starch at some speed $v$. (c) Measuring the water content of the starch at different times, either by destructive sampling [87] or x-ray tomography [114], demonstrates that the crack tips track a surface of constant moisture content, around a sharp drying front that slowly invades the starch mass.

highly permeable crack network [121-124]. The cooling, and hence cracking, proceeds via relatively well-defined fronts that slowly advance into the initially molten lava lake, which may take years to cool [121], and the cracks carve out the columnar features as they grow. Although more complex arrangements are possible [125], a typical lava flow will cool into two layers: an upper colonnade, growing from the exposed upper surface, and a lower colonnade, growing from the bottom of the flow [110]. A more disordered set of columns, or entablature, often replaces part, or all, of the upper colonnade, as in fig. 26(a), or forms between the upper and lower colonnade [110].

In lava, columns typically range from centimetres to metres across $[122,123]$, whereas in starch they are typically a few millimetres in size $[85,87]$. It is known from petrographic analysis of lavas that smaller columns tend to have cooled faster than larger ones $[123,125]$. Similar conclusions have been inferred by interpreting regular markings called striae [122,124,126], which resemble chisel-marks along the column faces and record the stickslip type of growth of individual cracks, as they carve out columns [126].

In corn starch the transport of moisture and the growth of columns can be monitored during drying, for example by x-ray tomography $[86,114,118]$, or by the destructive sampling of experiments [83,87], as shown in fig. 26(b). The initially wet and fluid starch slurry will dry evenly (i.e. without any significant gradients in moisture content, or stress), up to a point. During this time it solidifies and may shrink, but any cracks that form will break across the entire sample of starch (see e.g. $[79,127])$. When 
it reaches a water content of about $\phi_{\mathrm{w}}=0.25-0.3 \mathrm{~g} / \mathrm{cm}^{3}$, the main drying mechanism changes $[114,115]$. Corn starch granules are themselves porous, allowing for significant reservoirs of water to be stored in both the pores between grains, and the pores within grains [116]. At the critical $\phi_{\mathrm{w}}$, most of the water has been lost from between the grains, and liquid can no longer be efficiently moved by Darcy flow and capillary pressure gradients. Instead, it must be removed from within the sample by the much slower process of evaporation and vapour transport. This transport bottleneck leads to a second type of drying, in which a well-defined drying front invades the drying mass of starch $[115,116,128]$, as shown in fig. 26(c). A set of cracks grows away from the drying surface, tracking the drying front, and cutting out polygonal columns of starch as it advances.

The size of columnar joints reflects how quickly they cooled, or dried. A significant application of analogue experiments in starch was to show that this size is inversely proportional to the speed at which the cooling or drying front advances into the cracking body [87]. In both systems the stresses are confined to a thin layer around this front, where the crack tips are also located. For starch the uniform material ahead of the drying front means that there are no stress gradients there. Similarly, for lava the material ahead of the front is too yielding to support significant stresses. In both cases the material behind the front is already heavily fractured, by cracks that can accommodate any further contraction. For cracks in a thin stressed layer, it is known that the crack spacing $L$ will be proportional to the thickness of this layer, with a constant of proportionality of order one [81, 129-131]. The column size $L$ can thus be used as a natural lengthscale for describing the cooling or drying front.

As in eqs. (23) and (25), both heat and pore pressure (and, by extension, $\phi_{\mathrm{w}}$ ) are diffusive variables. In the reference frame that is moving along with a flat drying front, advancing at speed $v$ along a direction $z$, this leads to an advection-diffusion equation for moisture

$$
\partial_{t} \phi_{\mathrm{w}}=v \partial_{z} \phi_{\mathrm{w}}+\partial_{z}\left(D_{\mathrm{w}}\left(\phi_{\mathrm{w}}\right) \partial_{z} \phi_{\mathrm{w}}\right),
$$

where $D_{\mathrm{w}}$ is a concentration diffusivity [115], similar to the one in, eq. (4). $D_{\mathrm{w}}$ can be measured by inverting moisture profiles, e.g. from fig. 26(c) $[87,116]$. If the drying front is advancing steadily, such that the time-derivative is negligible, if we can approximate the diffusivity by a constant, $D_{\mathrm{w}}\left(\phi_{\mathrm{w}}\right)=D_{\mathrm{w} 0}$, and if all lengths are scaled by $L$, then eq. (26) simplifies to the dimensionless relation

$$
P e^{\prime} \partial_{z} \phi_{\mathrm{w}}+\partial_{z z} \phi_{\mathrm{w}}=0
$$

where

$$
P e^{\prime}=\frac{v L}{D_{\mathrm{w} 0}}
$$

is an effective Péclet number for moisture content, which should encode all the experimental parameters of how columns grow in drying starch.

Experiments have shown that cracking in starch proceeds with $L \sim v^{-1}$ at a constant $P e^{\prime}$ of about 0.1 [87],

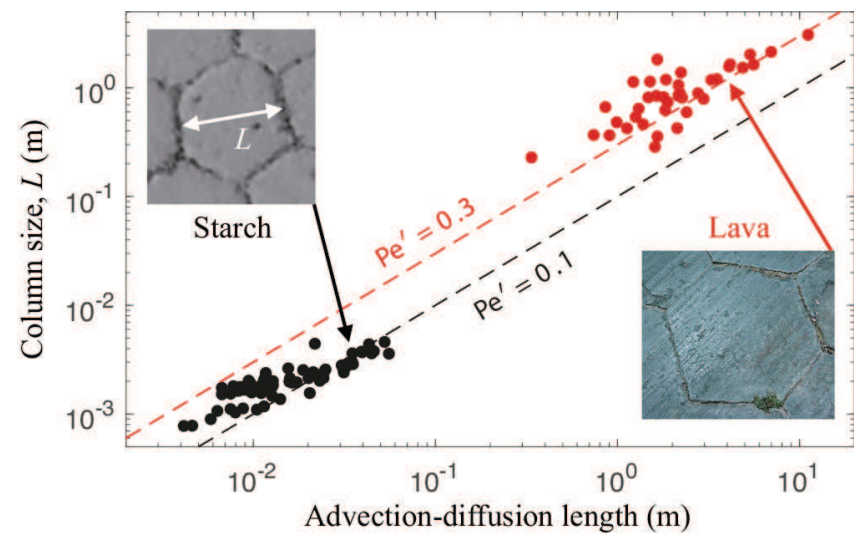

Fig. 27. The size of columnar joints in starch (black points) and lava (red points) scales with the advection-diffusion length, namely $D_{\mathrm{w} 0} / v$ for drying starch and $D_{T} / v$ for cooling lava. Data are from ref. [87].

as demonstrated in fig. 27. Detailed finite-element modelling of the cracking front allows for the possibility of a smaller exponent, for example $L \sim v^{-0.75}[118,132]$. However, a derivation similar to that of eqs. (26)-(28) can also be made for the thermal cooling of basalt [124], where the steady-state temperature gradients are known to closely match the solutions of the appropriate advection-diffusion problems [121]. Observations on columnar joints in lava flows from around the world, also given in fig. 27, show the same scaling as that of columns in dried starch, when the concentration diffusivity of water $\left(D_{\mathrm{w}}\right)$ is replaced by the thermal diffusivity $\left(D_{T}\right)$ of basalt $[87,124]$. The data collapse of the two different systems, when scaled with their advection-diffusion length, lends strong support the simple scaling arguments made above.

The patterns of starch columns can be imaged in full $3 \mathrm{D}$ by x-ray tomography $[86,114,115,118]$. This has allowed more detailed investigations of how they order than has been possible in the case of lava flows. The crosssectional pattern of polygonal columns can be described by metrics such as the relative distributions of the polygon areas, the numbers of their neighbours, their edge lengths, the angles between their edges, and so on [86]. Many of these metrics come from the study of 2D foams [133] and there are, indeed, similarities in the dynamics allowed in the two systems $[86,113,115]$. As they grow, columns can merge by a crack, defining the edge between them, failing to propagate. Alternatively, new columns can appear, typically from the vertices between three existing columns (like a time-reversed T2 process in foams [133]). Finally, adjacent pairs of columns can exchange an edge with each other (for foams a T1 process [133]), for example four hexagonal columns can swap an edge to become two pentagons, and two heptagons. These processes allow the topology of the pattern to evolve, and the scale of the columns to respond to changes in drying or cooling conditions [115]. By tracking individual columns through a colonnade, it can also be seen that there is a tendency to equalise the areas of nearby columns - a larger-thanaverage one will tend to shrink, whereas a small column 
will tend to grow rapidly, at the expense of its neighbours areas [115].

As a colonnade grows, the pattern of cracks is dynamic, according to the mechanisms just discussed. The cracks near the edges of a colonnade are less well ordered than those deep within it, in both lava [134] and starch [86]. If conditions change as the columns grow, they can also respond by changing size [86]. Alternatively, if conditions are held constant, the columns maintain a fixed average size, but individual cracks will still move around slightly, new columns will form, and old columns will merge, as discussed above [115]. In this quasi-static state, the pattern metrics of the starch match those of well-ordered basalt flows, like the Giant's Causeway [86, 135]. These ordering processes have been studied by a variety of sophisticated modelling techniques, such as phase-field modelling [136] and finite-element models incorporating crack growth [119].

Other than the examples focussed on here, columnar jointing has been reported in a variety of materials. Similar features have been reported in lunar basalt [137] and by satellite inspection of Martian crater walls [138], for example. They have also been shown to form in quenched glass [109], chemically laced vitrified ice [139], thermally shocked sandstone [140], and cooled stearic acid [141]. The most accessible experiments remain drying starches. Although this remarkably simple experiment has shown its versatility in explaining centuries-old questions $[105,106]$, there still remain several open areas of inquiry. For example, the disordered entablature [110] that is common in lava has never been recreated in desiccation cracks, and the reasons and criteria for its appearance in lava also remains ambiguous (other than their typically faster cooling rate, perhaps aided by flooding [125]). Similarly, lava columns occasionally show a wavy or "breathing" mode, where the faces of individual columns smoothly and sinuously advance and recede [124]. No reason for this is known, nor has it been reported in starches, although there is some similarity to wavy cracks seen in $2 \mathrm{D}$ directional cooling experiments $[142,143]$. Finally, although there has been much progress made on understanding the patterns of columnar joints, including what is discussed in the following section, no model or theory has been able to explain, convincingly and quantitatively, the observed metrics of the columnar patterns, such as the exact distributions of the areas or number of sides of the polygonal columns.

\subsection{Permafrost and evolving mud cracks}

If a homogeneous slurry or layer of paint is dried, cracks can open and break the film or coating up into pieces [79, $127,144,145]$. The contraction cracks will typically open one by one, and advance until they hit something, such as another crack. A crack will turn, or curve, to intersect a pre-existing crack at two right angles, as this allows it to release the most strain energy [79, 127]. As drying proceeds not only will the crack density increase, but earlier cracks will also accumulate more plastic strain, and

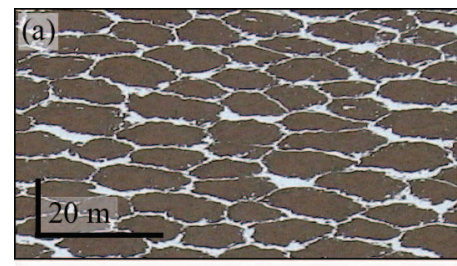

(c)

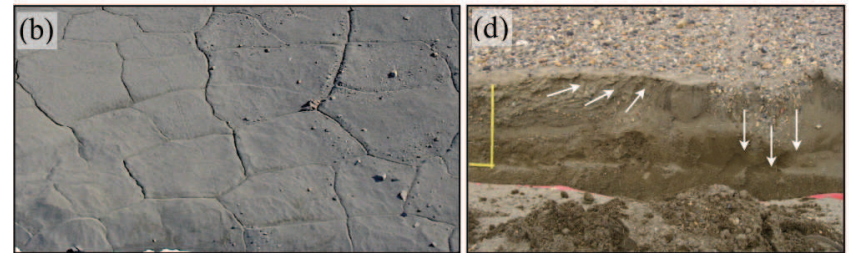

Fig. 28. Polygonal terrain is found in permafrost. (a) Wellordered patterns are seen in Beacon Valley, Antarctica. Here, a recent snowfall has highlighted the cracks. (b) At New Harbour, Antarctica, the pattern is younger and less ordered [147]. (c) Permafrost is permanently frozen soil. If cemented by ice it can support thermal stresses. In winter the ground cools and shrinks, and cracks open. These can be in-filled by surrounding soil and ice. In the summer the cracks partially heal, but remain weak; each winter's cracks are guided by the previous cycle [148]. (d) The injected material will eventually push up its surroundings, creating ridges around each crack, as indicated on this excavated permafrost crack.

can appear with a larger aperture, or opening $[145,146]$ The sequential subdivision of the film into smaller and smaller pieces will stop when the crack spacing is comparable to the thickness of the cracking layer [83, 127, 144], as the surface stresses in the material between adjacent cracks is then placed under compression, rather than tension $[130,131]$.

The situation just described is a good model for crack patterns with four-sided pieces, i.e. a rectilinear pattern. However, just like columnar joints, many different crack patterns show a more hexagonal planform, as in fig. 25. The most well known of these is polygonal terrain.

The ground in the polar regions of the Earth and Mars is permanently frozen, and huge areas of both planets are covered by networks of surface cracks, a few tens of meters across, as shown in fig. 28. This so-called polygonal terrain is the result of seasonal thermal stresses: the ground cools, shrinks and may crack in the winter months [147-149]. Cracks are typically found when the soil is rigidly held together, or cemented, by ice. While the cracks are open, debris such as sand or snow can fall into them. Thus, when the ground warms, the cracks will not fully heal, and a wedge of injected material will slowly build up in each crack. Over tens of thousands of years the resulting gradual motion of the crack, and its surroundings, can completely recycle and resurface the upper few meters of soil $[147,150,151]$. The crack pattern also slowly changes. Initially, it forms a rectilinear pattern dominated by cracks meeting at right angles $[147,151]$. Over many thousands of seasonal cycles, however, the pattern will shift into a more hexagonal symmetry $[82,147]$. The timescale here too long to be easily observed, although the gradual activity of 

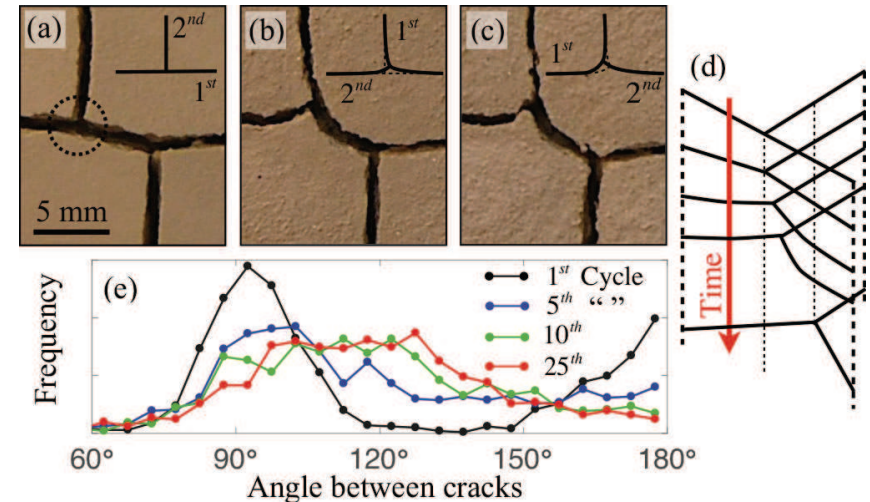

Fig. 29. Cracks in clay slurries evolve when repeatedly dried and rewetted. (a) When a homogeneous slurry dries, cracks open sequentially, with earlier cracks intersecting later ones at right angles. An overlaid sketch shows how, at the circled intersection, the horizontal crack opened before the vertical one. In (b) the second, (c) the third and subsequent cycles of cracking the crack positions are similar to their first appearance. However, the sketches show how the order of opening changes, allowing for small differences to accumulate. (e) Over time the intersections evolve, to favour $120^{\circ}$ angles between cracks. (e) Data showing this ordering process over 25 cracking cycles in bentonite clays are provided from ref. [82]

some sites has been monitored over decades-long studies $[147,148,150,151]$.

Analogue experiments in dried clays have explored the dynamics of this process, and have showed how an initially rectilinear pattern can evolve towards a hexagonal one $[82,83]$. Mimicking the seasonal stress cycles of permafrost, a clay slurry can be repeatedly dried, cracked, and then rewet to heal the cracks. The first time it dries, the slurry cracks with the expected rectilinear pattern, and the sequence of crack openings is reflected in the geometry of the pattern (i.e., later cracks intersect earlier ones at right angles) [83]. When rewet the cracks close. There remains some memory of these cracks in the clay (e.g., through incomplete healing, microvoids, or variations in density), and they act as lines of weakness that guide cracks in later drying cycles. However, the sequence at which the cracks appear in each cycle is different, and so the paths that maximise the strain energy release rates of the growing cracks will also be different [82]. As demonstrated in fig. 29, this creates the opportunity for the positions and angles around crack intersections to evolve. Specifically, any intersection, or vertex, between three cracks will slowly travel along the direction of the crack that had originally approached that vertex at right angles, and the angles around this intersection will relax to $120^{\circ}[82,83]$.

In order to evolve towards a hexagonal symmetry, a planar crack network should have some recurring cycle of cracking and healing, with enough memory of the crack positions between cycles to guide the next generation of cracks [82]. These conditions are very general, and apply to both the drying clay experiments and polygonal terrain. More recent freeze-thaw experiments in clays also capture a similar process [152]. Furthermore, a related situation is seen in crack patterns in some gypsum sand dunes, where moisture cycles stress a surface layer of weakly concreted sand. The sand is slowly eroded away by wind, allowing the crack network in this rigid crust to advance over time, and evolve as it does so [153]. Finally, the skin on the heads of embryonic crocodiles is argued to crack in similar ways, with the cracked tiles developing into scales [103].

In addition to helping explain a broad range of geophysical and biological phenomena, understanding how to change a crack pattern has potential engineering applications in creating designer materials. As an example of this, repeated wetting-drying cycles have been used to improve the homogeneity and connectivity of crack networks that are then used to template the deposition of transparent conductors or electrodes in thin films [154].

\subsection{Cracks over patterned substrates}

Nature rarely provides as simple a situation as a flat and uniform layer. Cracks over different substrates can reflect the shapes of any underlying features, as shown in fig. 30. Variations in the substrate can be engineered to nucleate, halt, bend or redirect cracks in a layer above them [155157]. Art can be made by creative control over this, as in the works of Andy Goldsworthy [158]. In geophysics, attempts have also been made to interpret 'ghost craters' in satellite images, where superficial cracks arrange into clear crater-like rings $[159,160]$.

Drying thin films offer a simple means of studying the interaction of an uneven substrate with a cracking layer. A recent work in this direction has looked at cracks over corrugated, or rippled substrates [161]. The authors showed that the resulting crack patterns only depend on the dimensionless ratios of the geometric lengthscales in the problem. In their simple configuration these lengths were the thickness of the cracking layer, and the characteristic amplitude and wavelength of the substrate relief. As demonstrated by fig. 30(d)-(f) the cracks in these experiments showed transitions between three patterns: wavy cracks that were guided by an underlying ridge, but which alternated between the two sides of it; a linear array of cracks growing directly over each buried ridge, with later cracks joining up the spaces between them to form a ladder-like structure; and isotropic cracks which effectively ignored the ridges.

The transitions between these patterns can be explained by identifying the crack locations and paths which maximise the difference between the strain energy released by a growing crack, and the energy spent creating the new surface area of that crack [161]. For the geometries that create wavy cracks there are two energetically favourable positions, to either side of a buried ridge. The growing cracks appear to oscillate between them. For the ladderlike cracks, the most favourable crack locations are directly over the buried ridges. Furthermore, the strength of alignment in the crack network can be characterised by order parameters, similar to the liquid-crystal order parameter, as the cracks are long rod-like features. The 


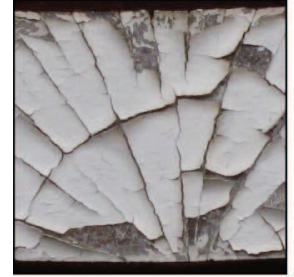

(a) Paint on wood

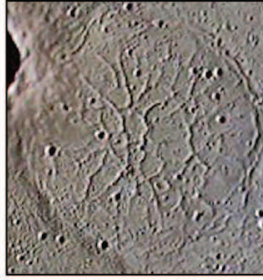

(b) Buried crater

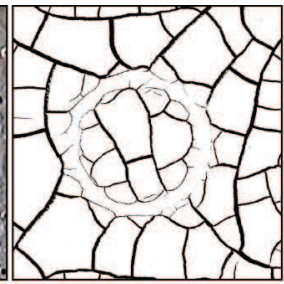

(c) Buried ring

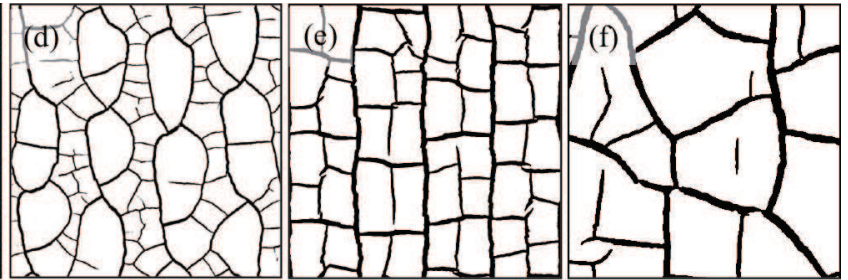

$\sim \sim 2 \%$

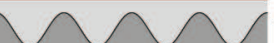

Fig. 30. Crack networks are influenced by what lies beneath them. Examples in nature include (a) cracks in paint on a wooden beam, which reflect the tree rings in the wood below, and (b) ghost craters, where cracks and graben reflect the shape of the underlying terrain [160]. (Image Credit: NASA/Johns Hopkins University Applied Physics Laboratory/Carnegie Institution of Washington.) These patterns can be studied by drying slurries over textured substrates. For example, (c) a buried ring creates similar features to a ghost crater. (Image courtesy of T.-E. Regenhardt.) When clays are dried over a corrugated substrate, they can show (d) wavy, (e) ladder-like or (f) isotropic crack patterns, depending on the ratios of the layer thickness to the amplitude and wavelength of the underlying ripples [161].

strongest ordering was found to occur when the thickness of the cracking layer was equal to, or slightly smaller than, the wavelength of the substrate relief.

\section{Cooling dynamics of "soft" planets}

\subsection{Convection in planetary mantles}

As a planet forms hot in a cold universe, its whole subsequent evolution is dictated by its cooling. In turn the latter strongly depends on the convective regime prevailing in its most viscous enveloppe. In a rocky planet like the Earth, convective motion occurs in the $2900 \mathrm{~km}$ thick layer of solid rocks under our feet, which is called "mantle" (fig. 1(h)). Today we observe radically different convective regimes on the Earth and its two "sister" planets, Venus and Mars [162]. For example, Earth's has numerous "plates" that drift across its surface; $~ 60 \%$ of these plates (primarily in the oceans) are renewed quasi-continuously by "subduction" (where plates sink into the mantle) and "accretion" at mid-ocean ridges (where the plates spread apart and new plate is created). In contrast, the surface of Mars is very old and plate tectonics stopped there at least 4000 million years (hereafter "Myr") ago. Venus presents an intermediate case, with most of its surface younger than 300 to $1000 \mathrm{Myr}$ old; and it has been proposed that episodic foundering of Venus' plates causes resurfacing of the whole planet in a single event. The conditions necessary to select one of the above regimes or the other remain strongly debated. The difficulty comes from the complexity of rocks rheology, which depends on temperature, pressure, strain rate, damage and composition. Of these parameters, rock rheology is most sensitive to temperature, rocks being viscous at high temperature and on long timescale (i.e. $100 \mathrm{Myr}$ ), but brittle at low temperature and short timescales (i.e. earthquakes). This "soft matter" behaviour can be recovered in the laboratory in colloidal dispersions, whose rheology varies from viscous to visco-elasto-plastic to brittle when the ionic force or the particle volume fraction increase (see sect. 2). The above properties of colloidal fluids closely mimic the rocky planets and, thus, were the ideal fluids for laboratory experiments of thermal and solutal convection. Understanding the physical evolution of these systems provides insights into the dynamics of planetary interiors.

Cooling planetary mantle or drying of a thick layer of colloids will form a cold plate or a gelled skin on the layer surface, respectively, if the Péclet number is large enough:

$$
P e=\frac{Q_{S} h}{D_{T}}=\frac{V_{E} h}{D_{c}} \gg 1,
$$

where $h$ is the fluid thickness, $Q_{S}$ is the planetary surface heat flux, $\Delta T$ the temperature difference across the mantle, $V_{E}$ is the laboratory evaporation rate, $D_{c}$ is the chemical diffusivity. A planetary mantle, and all our experiments are running in this limit.

Several other parameters are needed to fully describe convection in a complex fluid. The most important is the Rayleigh number, $R a$, which controls the intensity of convection; $R a$ compares the driving buoyancy force to the resisting effects of diffusion and viscous dissipation:

$$
R a(h, \nu)=\frac{\alpha \Delta T g h^{3}}{D_{T} \nu}=\frac{\Delta \rho g h^{3}}{\rho D_{c} \nu},
$$

where $g$ is the gravity acceleration, and $\nu$ is the kinematic viscosity of the bulk fluid. In the drying case, the density contrast driving convection $\Delta \rho$ is due to heterogeneities in the particle volume fraction. For a cooling planetary mantle, the density contrast is equal to $\rho \alpha \Delta T$ where $\alpha$ is the thermal expansivity, and $\rho$ its reference density. Convection occurs when $R a$ is greater than a critical value $R a_{c} \sim 1000$ [163]. $R a$ ranges between $10^{6}$ and $10^{9}$ for planetary mantles, and $10^{4}-10^{11}$ for the laboratory. When the viscosity depends strongly on temperature or on particle volume fraction, the convective regime depends also on the maximum viscosity ratio $\gamma=\nu($ surface $) / \nu($ bulk $)$ present in the system. When $\gamma \geq 10^{4}$, the "stagnant lid" regime prevails whereby convective motions develop below a stagnant viscous sublayer located at the surface, forming a "plate" (e.g., ref. [164] for a review). 

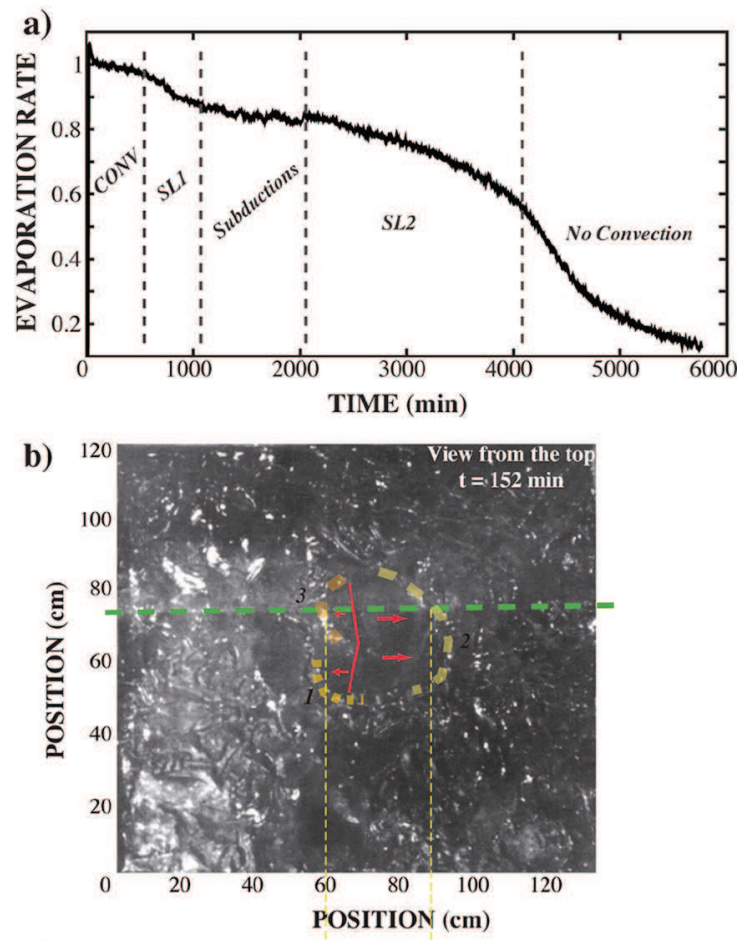

c)

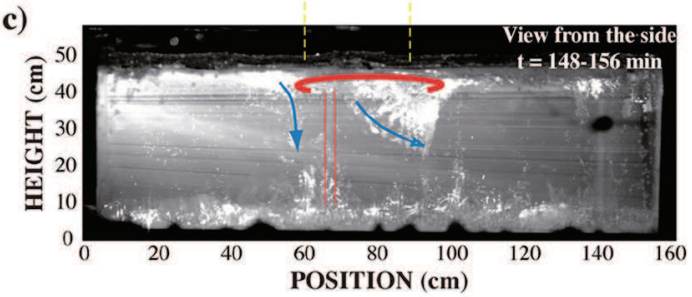

d)

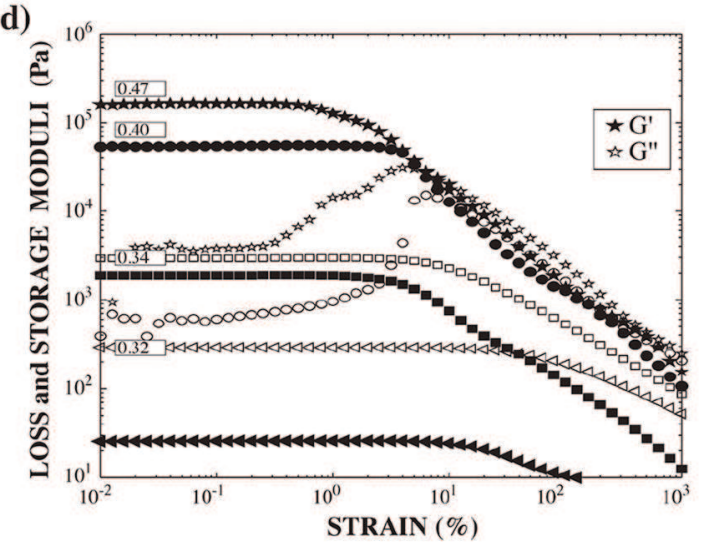

Fig. 31. Typical evolution of a drying experiment. (a) Evaporation rate (normalized by the corresponding evaporation rate in pure water in the same setup) through time showing the different regimes. (b) Top view of the tank showing skin buckling, and its response to the impact of a hot upwelling. (c) Side view showing time-lapse photography of skin subduction induced by the plume (adapted from [165]). (d) Rheometry of Ludox@HS40. Loss and storage moduli as a function of the oscillatory strain amplitude for different particle volume fractions $\phi$. The dispersion becomes more elastic as $\phi$ increases. The linear elasticity domain for $\phi \geq 40 \%$ breaks down when the local stress becomes greater than a critical value (adapted from $[4])$.

\subsection{Gel formation by evaporation to investigate subduction processes and planetary evolution}

How convection can break a surface plate and initiate subduction remains one of the most difficult questions of geodynamics [166]. Drying Ludox@ layers exhibit spontaneous development of subduction, and at least two different mechanisms can be at play.

First, let us consider a layer of Ludox $(\mathrm{R}$ which is dried at a constant temperature from above. The time-evolution of the evaporation rate $V_{E}$ (fig. 31(a)) then presents five main regimes. When the water evaporates at the free surface, the volume fraction of silica particles $\phi$ increases and the total fluid layer thickness $h$ decreases with time $t$. Since $P e$ is large, $\phi$ increases near the free surface forming a boundary layer (SBL) whose thickness $\delta$ increases with time [167]. However, if the local $\operatorname{Ra}(\delta, \nu)$ becomes greater than $R a_{c}$, the SBL becomes gravitationally unstable and the surface will continously and completely rejuvenate. This first regime of vigorous convection ("CONV" in fig. 31(a)) is characterized by a nearly constant evaporation rate. Micro-meter sized particles sprinkled on the surface undergo strong motions and are quickly engulfed within the fluid layer. Shadowgraphs show the formation of plumes descending from the surface. However, $\phi$ is steadily increasing through time until at the surface it reaches a critical value $\phi_{g}$, where the silica dispersion becomes a gel and a skin phase is formed [102,167]. Gel formation occurs in the second regime "SL1" in fig. 31(a), where $V_{E}$ decreases significantly. Particles sprinkled on the drying surface remain immobile, showing that indeed a gelled skin is developing. Shadowgraphs continue to show active convection in the bulk of the fluid, i.e. underneath the skin. The SL1 regime corresponds to the "Stagnant Lid" regime described in the previous section. The evaporation-driven flow of solvent through the skin generates pressure gradients and therefore, also in-plane stresses in the skin $[168,169]$. As a result, the surface skin starts to visco-elastically buckle (fig. 31(b) $[102,169,170]$ ), forming wrinkles with an amplitude that grows with time. As the wrinkles grow, the strain and stress in the skin increase until the system leaves the elastic regime (fig. 31(d)) and plasticity kicks in. Eventually, part or most of the surface skin founders into the fluid. Usually the foundering is onesided, i.e. the skin breaks along one wrinkle but only one side of the tear plunges into the fluid layer, while fluid from the tank bulk upwells to the surface. Therefore the "trench", i.e. the location where the surface skin plunges into the fluid, moves towards the remaining skin through time ("trench roll back", fig. 31(c)). Moreover, once new fluid has reached the surface, it starts drying, developing a new skin until buckling and subduction are repeated. Therefore, this third regime ("Subductions" in fig. 31(a)) presents a strong episodicity. While $V_{E}$ remains quite stable on the long-term, it presents more locally a succession of decreases (skin formation) and rises (subduction and upwelling of less viscous fluid). These subductions possess some striking similarities with Earth's subduction, e.g. one-sided and trench roll-back, which are never seen 
in purely viscous fluids $[4,171]$. However, we cannot yet speak about continuous "plate tectonics" like on Earth.

Another way to trigger subduction is the impingement of a rising hot plume on the underside of the skin when the system is also heated from below. The upwelling plume produces tensile fractures in the skin, through which buoyant plume material rises before spreading above the skin. The weight of this growing gravity current then leads to bending and eventual subduction of the skin along arcuate segments. Subduction is energetically costly due to three-dimensional tearing along its edges and, thus, generally stops when plume spreading stops. Scaling analysis suggests that this regime with limited, plume-induced subduction would best develop in the hot conditions of early Earth or present-day Venus [165]. Indeed, the observed semi-circular shape of the experimental subduction segments are analogous to those seen at two proposed sites of plume-triggered subduction at Quetzalpetlatl and Artemis coronae [172]. Other experimental deformation structures and subsurface density variations are also consistent with topography, radar, and gravity data for Venus.

But in all cases, fig. 31(a) further shows that as the system continues to dry (or the planet to cool), it transitions once more to a stagnant lid regime ("SL2"), where the skin has now become too stiff and thick to break. $V_{E}$ smoothly decreases through time as the surface skin thickens [167]. Shadowgraphs show that small-scale convective motions are still present underneath the skin surface. This regime probably reflects Mars dynamics today: Mars is smaller than Earth or Venus and it has already cooled significantly, placing it in a further advanced state of its evolution [162].

The last regime ("No Convection" in fig. 31(a)) occurs when the skin thickening reaches the base of the tank. Then, the evaporation rate decreases dramatically towards 0 , as cracks open and the porous skin desaturates. The "No Convection" regime corresponds to the situation described in detail in sect. 7 .

\subsection{Gel formation by salt diffusion to investigate plate accretion at mid-ocean ridges}

On Earth, new plates are created at mid-ocean ridges ("MOR"). This accretion process involves complex interactions between magmatic intrusion, cooling, and faulting at the ridge axis as the two plates spread apart at rates between between 0.5 and $15 \mathrm{~cm} / \mathrm{yr}$ (fig. 32), which depends strongly on the subduction occurring at the other end of the plates (fig. 1(h)). MOR axes are not straight, but segmented over a large range of scales from small bends (few kilometers wide) to large-offset (tens to hundreds of kilometers) transform faults (fig. 33 [173-175]). Both observations and modeling suggest that variations in axial morphology and segmentation strongly depend on the local stress regime, and therefore on i) the plate axial mechanical properties, ii) the plate axial thermal structure, which is function of mantle temperature and spreading rate, and iii) magma supply to the axis. All three of which influence each other. Due to this complexity, the processes that control segmentation are still largely debated [176-183].
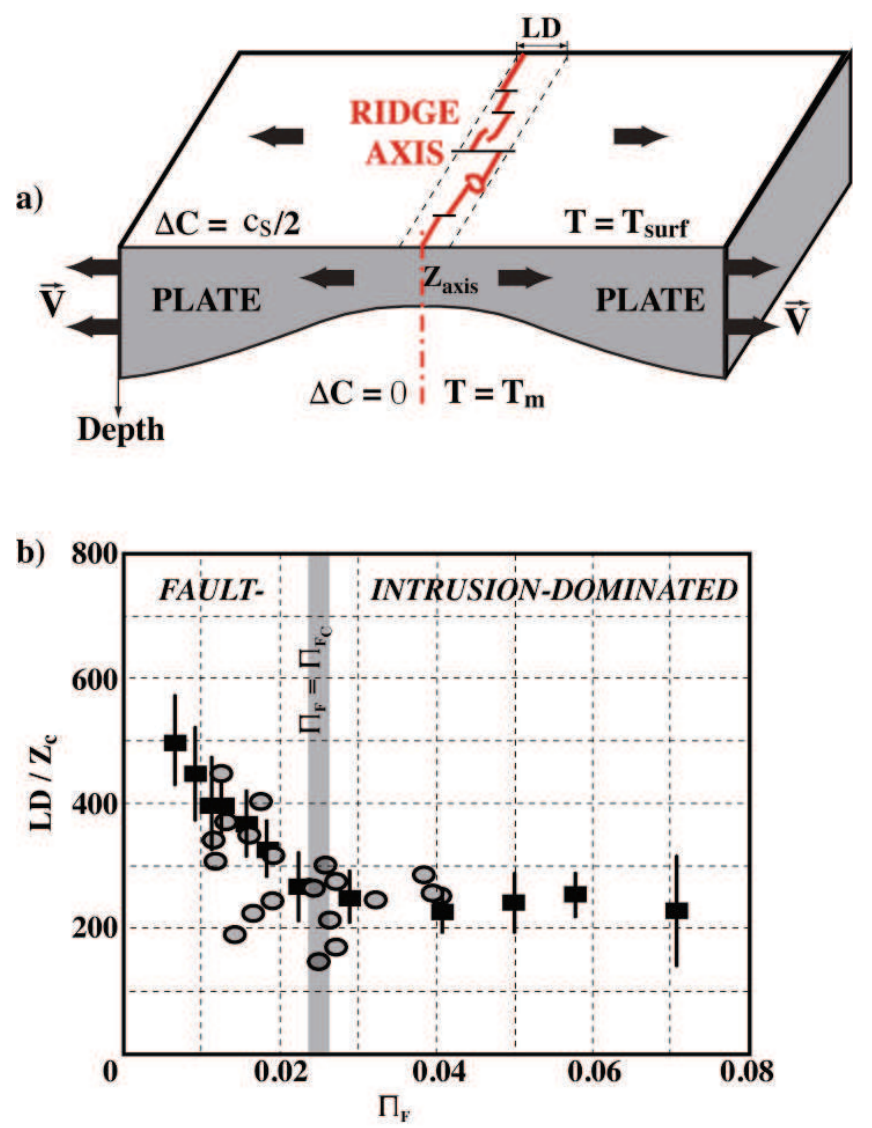

Fig. 32. (a) Cartoon showing the growth of the lab or mantle plate by salt or thermal diffusion respectively, while it is being pull apart at velocity $V$. The spreading velocity is perpendicular to the initial ridge axis. (b) Lateral deviation of the ridge axis $L D$, normalized by the critical axial thickness $Z_{C}$, as a function of the axial failure parameter $\Pi_{F}$. The black squares are the laboratory experiments and the grey circles are the oceanic data. Adapted from [184].

Accretion experiments using Ludox $($ ) dispersions provide a useful tool to quantitatively study the spreading processes as Ludox(R's mechanical properties are well known $[4,75]$ and these dispersions allow independent variation of spreading velocity and mechanical structure of the plate while permitting axial fluid intrusion to occur. To take advantage of these properties for simulating ridge spreading, a Ludox $\cap$ layer is put in contact with a saline solution of concentration $c_{s}$. A white skin forms nearly instantaneously if the $\mathrm{NaCl}$ concentration at the interface is greater than a critical value $c_{s}^{c}$ [75]. Saline diffusion across the interface yields a constant interface concentration of $c_{s} / 2$, which drives skin growth by salt diffusion through time. This is analogous to the cooling of a planet surface plate by thermal diffusion. The newly formed plate is attached to two plexiglas plates moving apart at a constant velocity $V$ (perpendicular to the initial axis), which results in the localization of accretion and fracture on a roughly linear axis (figs. 32 and 33). The axial plate thickness $Z_{\text {axis }}$ is therefore controlled by a balance of advection 


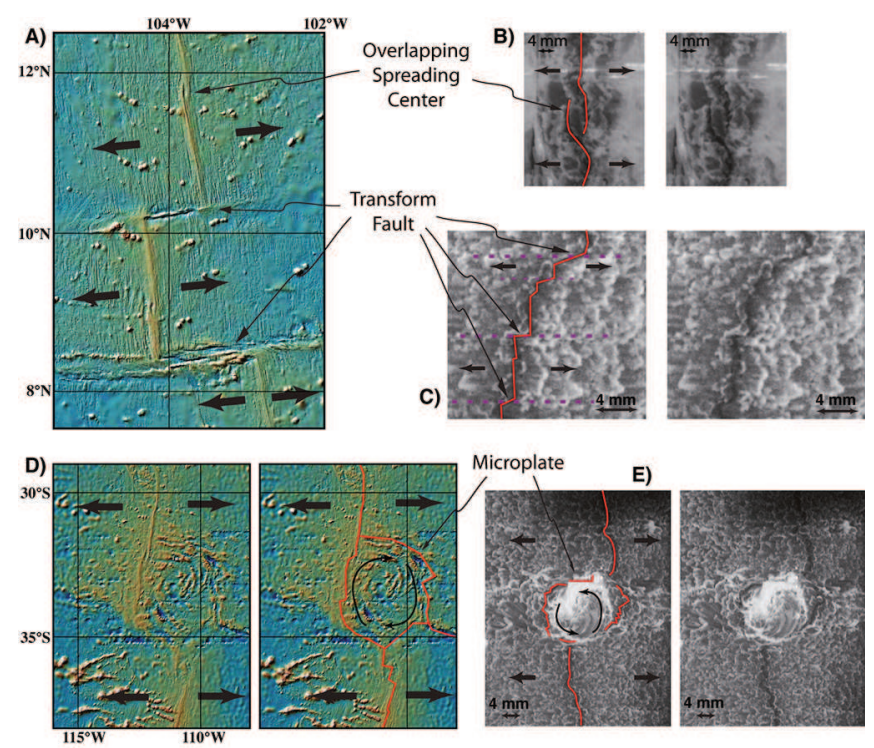

Fig. 33. Typical mechanical instabilities encountered both on the mid-ocean ridges (A) and (D), and in the laboratory experiments (B), (C) and (E). On the bathymetric maps (A) and (D) drawn using http://www.marine-geo.org/rmbs/-, seafloor depth increases from orange to blue. The accretion axis have been highlighted in red when needed. The arrows show the direction of spreading.

and diffusion:

$$
Z_{\text {axis }}=-\frac{2 D}{V} \log \left(\frac{\Delta C}{c_{s} / 2}\right)=-\frac{2 D}{V} \log \left(\frac{T_{c}-T_{m}}{T_{0}-T_{m}}\right),
$$

where $D$ is the chemical (or thermal) diffusivity and $T_{c}$ the temperature below which the lithosphere is elasticbrittle. For the salt concentrations used $(2.7-6.15 \mathrm{~mol} / \mathrm{l})$, skin forms nearly instantaneously and the axis is always solid. Separately changing surface water salinity and plate spreading velocity allows independent examination of the role of spreading and axial plate thickness on the evolution of the system.

Figure 33 shows that the shape of the ridge axis observed in the laboratory experiments is quite similar to the terrestrial case. In particular, morphologies of segmentation such as overlapping spreading centers, transform faults and rotating microplates are commonly observed. Moreover, accumulation of these offsets causes the ridge axis to have a tortuous geometry as is indeed observed on Earth (fig. 1(h)). A quantitative comparison is possible when we introduce the "axial failure parameter", which describes the controls on material deformation and failure [184]:

$$
\Pi_{F}=\frac{K_{1 c}}{\sigma_{Y} Z_{\text {axis }}^{1 / 2}}=\sqrt{\frac{Z_{m}}{Z_{\text {axis }}}},
$$

where $K_{1 c}$ is the fracture toughness and $\sigma_{Y}$ is the yield stress marking the boundary between brittle and plastic (ductile) behavior. $Z_{m}=\left(K_{1 c} / \sigma_{Y}\right)^{2}$ can be interpreted as the characteristic mechanical lengthscale below which plasticity becomes dominant in fracture processes. So when $\Pi_{F}$ becomes larger than a critical value $\Pi_{F c}$, the predominant behavior of the axis changes from brittle to plastic. Thus the deformation style strongly depends on $Z_{a x i s}$, which, in turn, depends on saline concentration (mantle temperature) and spreading rate: faster spreading or lower salinity (higher mantle temperature) yield smaller $Z_{\text {axis }}$ values (i.e., thinner mechanically strong axial plate). The value $\Pi_{F c}$ corresponds to a critical axial elastic thickness $Z_{c}=Z_{m} / \Pi_{F c}^{2}$, that only depends upon the mechanical properties of the material. Close-up examination of the experiments shows that the low- $\Pi_{F}$ regime seems dominated by fractures propagating from the surface down, while at higher- $\Pi_{F}$, accretion is dominated by intrusion of fresh fluid rising from below. These two end-member regimes have also been observed on Earth (e.g., Buck et al. [179]). Moreover, the lateral deviation $L D$ of the ridge axis (compared to a perfectly straight axis) presents two distinct trends as a function of $\Pi_{F}: L D$ values first decrease with increasing $\Pi_{F}$ until reaching a minimum after which the trend becomes flat. Comparison of laboratory and natural cases shows that the two data sets obey the same law when plotted as a function of $Z_{c}$ (typically $800 \mathrm{~m}$ on Earth, and $4 \cdot 10^{-5} \mathrm{~m}$ for Ludox $\AA$ ) and $\Pi_{F}$ (fig. 32(b)). So $\Pi_{F}$ seems to capture the most important aspects of ridge evolution.

\section{Conclusion}

This review presents a wide variety of problems from the formation of thin coatings to phenomena that occur at planets surface. The diversity of these processes lies in the extremely large size and time scales. Being able to propose experimental modelling to highlight, isolate and then analyse mechanisms of interest appear to be a great challenge. The results presented in this review attest of the reliability of laboratory experiments using the drying of complex fluids to reproduce and to study original mechanisms. One common element to all these approaches is to describe experimentally and numerically out-of-equilibrium systems involving multicomponent objects that strongly interact each other at small scales via physico-chemical surface interactions or mechanically through deformation. This diversity of interactions is responsible for the macroscopic properties of the materials after drying. The success of this experimental modelling is based on tunable inter components forces and the diversity of physical properties emerging during the drying process. The number and nature of components involved make the analogue approach a powerful method.

This collective work largely benefited from meetings within the French working group GDR CNRS 2980 "Approches Multiphysiques pour les Colloides Concentrés" (AMC2) being continued in GDR CNRS 2019 "Solliciter LA Matière Molle" (SLAMM) that groups a large number of the authors.

\section{Author contribution statement}

All the authors were involved in the preparation of the manuscript. All the authors have read and approved the final manuscript. 


\section{References}

1. Ioannis Gergianakis, Mise en oeuvre de nano-réservoirs anti-corrosion dans des procédés de traitement de surface, PhD Thesis, Université de Toulouse, Université Toulouse III-Paul Sabatier, 2016.

2. Frédérique Giorgiutti-Dauphiné, Ludovic Pauchard, J. Appl. Phys. 120, 065107 (2016).

3. B.V. Derjaguin, L. Landau, Acta Physicochim. URSS 14, 633 (1941).

4. E. Di Giuseppe, A. Davaille, E. Mittelstaedt, M. François, Rheol. Acta 51, 451 (2012).

5. David W. Richerson, Douglas W. Freitag, Technical Report, Oak Ridge National Laboratory.

6. https://en.wikipedia.org/wiki/ceramic\#/media/ file:si3n4bearings.jpg.

7. Joaquim Li, Bernard Cabane, Michael Sztucki, Jérémie Gummel, Lucas Goehring, Langmuir 28, 200 (2011).

8. G.K. Batchelor, J. Fluid Mech. 41, 545 (1970).

9. Prabhu R. Nott, Elisabeth Guazzelli, Olivier Pouliquen, Phys. Fluids 23, 043304 (2011).

10. Jeffrey F. Morris, Fabienne Boulay, J. Rheol. 43, 1213 (1999).

11. Martin Frank, Douglas Anderson, Eric R. Weeks, Jeffrey F. Morris, J. Fluid Mech. 493, 363 (2003).

12. Denis Semwogerere, Jeffrey F. Morris, Eric R. Weeks, J. Fluid Mech. 581, 437 (2007).

13. Yannick Hallez, Ioannis Gergianakis, Martine Meireles, Patrice Bacchin, J. Rheol. 60, 1317 (2016).

14. Aurore Merlin, Jean-Baptiste Salmon, Jacques Leng, Soft Matter 8, 3526 (2012).

15. B. Cuq, E. Rondet, J. Abecassis, Powder Technol. 208 , 244 (2011) (Special Issue: Papers presented to the Symposium STPMF 2009, Science and Technology of Powders and Sintered Materials).

16. J. Burgain, C. Gaiani, C. Cailliez-Grimal, C. Jeandel, J. Scher, Innov. Food Sci. Emerg. Technol. 19, 233 (2013).

17. D.E. Walton, C.J. Mumford, Chem. Eng. Res. Des. 77, 21 (1999).

18. Jose Miguel Aguilera, J. Food Eng. 67, 3 (2005) ( IV Iberoamerican Congress of Food Engineering (CIBIA $I V))$.

19. G.V. Barbosa-Cánovas, P. Juliano, Compression and Compaction Characteristics of Selected Food Powders, in Advances in Food and Nutrition Research, Vols. 233-307 (Academic Press, 2005).

20. Albert H.L. Chow, Henry H.Y. Tong, Pratibhash Chattopadhyay, Boris Y. Shekunov, Pharm. Res. 24, 411 (2007).

21. B. Adhikari, T. Howes, B.R. Bhandari, V. Truong, Int. J. Food Prop. 3, 323 (2000).

22. Nan Fu, Meng Wai Woo, Xiao Dong Chen, Dry. Technol. 30, 1771 (2012).

23. Xuelian Chen, Volodymyr Boyko, Jens Rieger, Frank Reinhold, Bernd Reck, Jan Perlich, Rainer Gehrke, Yongfeng Men, Soft Matter 8, 12093 (2012).

24. L. Pauchard, Y. Couder, Europhys. Lett. 66, 667 (2004).

25. Kyle Anthony Baldwin, Manon Granjard, David I. Willmer, Khellil Sefiane, David John Fairhurst, Soft Matter 7, 7819 (2011).

26. Céline Sadek, Herve Tabuteau, Pierre Schuck, Yannick Fallourd, Nicolas Pradeau, Cecile Le Floch-Fouere, Romain Jeantet, Langmuir 29, 15606 (2013).
27. Celine Sadek, Pierre Schuck, Yannick Fallourd, Nicolas Pradeau, Cecile Le Floch-Fouere, Romain Jeantet, Dairy Sci. Technol. 95, 771 (2015).

28. Celine Sadek, Pierre Schuck, Yannick Fallourd, Nicolas Pradeau, Romain Jeantet, Cecile Le Floch-Fouere, Food Hydrocolloids 52, 161 (2016).

29. Jacob Bouman, Paul Venema, Renko J. de Vries, Erik van der Linden, Maarten A.I. Schutyser, Food Res. Int. 84, 128 (2016).

30. Nan Fu, Meng Wai Woo, Xiao Dong Chen, Colloids Surf. B: Biointerfaces 87, 255 (2011).

31. Cécile Sadek, Huashan Li, Pierre Schuck, Yannick Fallourd, Nicolas Pradeau, Cécile Le Floch Foueré, Romain Jeantet, Dry. Technol. 32, 1540 (2014).

32. Sean Xu QI Lin, Xiao Dong Chen, Chem. Eng. Res. Des. 80, 401 (2017).

33. Nan Fu, Meng Wai Woo, Sean Xu Qi Lin, Zihao Zhou, Xiao Dong Chen, Chem. Eng. Sci. 66, 1738 (2011).

34. Xiao Dong Chen, Dry. Technol. 26, 627 (2008).

35. X.D. Chen, A. Putranto, Modeling Drying Processes - A Reaction Engineering Approach, 1st edition (Cambridge University Press, 2013) ISBN 978-1-107-01210-3.

36. Aditya Putranto, Xiao Dong Chen, Int. J. Food Prop. 19, 1726 (2016).

37. Xiao Dong Chen, Sean Xu Qi Lin, AIChE J. 51, 1790 (2005).

38. Sean Xu Qi Lin, Xiao Dong Chen, Dry. Technol. 24, 1329 (2006).

39. Sean Xu Qi Lin, Xiao Dong Chen, Chem. Eng. Process. 46, 437 (2007) Advances in the Application of Chemical Engineering Principles in Food Industry.

40. Sean Xu Qi Lin, Xiao Dong Chen, Dry. Technol. 27, 1028 (2009).

41. Kamlesh Patel, Xiao Dong Chen, Romain Jeantet, Pierre Schuck, Dairy Sci. Technol. 90, 181 (2010).

42. Peng Zhu, Kamlesh Patel, Sean Lin, Serge Méjean, Eric Blanchard, Xiao Dong Chen, Pierre Schuck, Romain Jeantet, Dry. Technol. 29, 419 (2011).

43. Yan Jin, Xiao Dong Chen, Dry. Technol. 27, 371 (2009).

44. Yan Jin, Xiao Dong Chen, Dry. Technol. 27, 1018 (2009).

45. Yan Jin, Xiao Dong Chen, Dry. Technol. 28, 960 (2010).

46. Yan Jin, Xiao Dong Chen, Int. J. Therm. Sci. 50, 615 (2011).

47. Alexander F. Routh, William B. Zimmerman, Chem. Eng. Sci. 59, 2961 (2004).

48. Robert D. Deegan, Olgica Bakajin, Todd F. Dupont, Greb Huber, Sidney R. Nagel, Thomas A. Witten, Nature 389, 827 (1997).

49. R.D. Deegan, O. Bakajin, T.F. Dupont, G. Huber, S.R. Nagel, T.A. Witten, Phys. Rev. E 62, 756 (2000).

50. Mohan Srinivasarao, David Collings, Alan Philips, Sanjay Patel, Science 292, 79 (2001).

51. Hideto Matsuyama, Stephane Berghmans, Douglas R. Lloyd, Polymer 40, 2289 (1999).

52. Hideto Matsuyama, Masaaki Teramoto, Ryo Nakatani, Taisuke Maki, J. Appl. Polym. Sci. 74, 159 (1999).

53. Masato Yamamura, Takatoshi Nishio, Toshihisa Kajiwara, Kitaro Adachi, Chem. Eng. Sci. 57, 2901 (2002).

54. C.S. Tsay, A.J. McHugh, J. Membr. Sci. 64, 81 (1991).

55. J. Fichot, R. Heyd, C. Josserand, I. Chourpa, E. Gombart, J.-F. Tranchant, M.-L. Saboungi, Phys. Rev. E 86, 6 (2012). 
56. G. Lelong, R. Heyd, G. Charalambopoulou, T. Steriotis, A. Brandt, K. Beck, M. Vayer, D. Price, M.-L. Saboungi, J. Phys. Chem. C 116, 9481 (2012).

57. R. Heyd, A. Rampino, B. Bellich, E. Elisei, A. Cesaro, M.-L. Saboungi, J. Chem. Phys. 140, 124701 (2014).

58. F. Doumenc, B. Guerrier, C. Allain, Europhys. Lett. 76, 630 (2006).

59. Béatrice Guerrier, Charles Bouchard, Catherine Allain, Christine Bénard, AIChE J. 44, 791 (1998).

60. Zhiyon Gu, Paschalis Alexandridis, J. Pharm. Sci. 93, 1454 (2004).

61. Christina Ratti (Editor), Advances in Food Dehydration, Contemporary Food Engineering Series (CRC Press, 2009).

62. M. Thiriet, Biology and Mechanics of Blood Flows, Part II: Mechanics and Medical Aspects (Springer, 2008).

63. W. Bou Zeid, D. Brutin, Colloids Surf. A 430, 1 (2013).

64. L. Pauchard, Europhys. Lett. 74, 188 (2006).

65. F. Smith, C. Nicloux, D. Brutin, Phys. Rev. Fluids 3 013601 (2018).

66. F. Smith, N. Buntsma, D. Brutin, Langmuir 34, 1143 (2018).

67. K. Range, F. Feuillebois, J. Colloid Interface Sci. 203, 16 (1998).

68. T. Stotesbury, M.C. Taylor, M.C. Jermy, J. Forensic Sci. 62, 74 (2017).

69. N. Laan, F. Smith, C. Nicloux, D. Brutin, Forensic Sci. Int. 267, 104 (2016).

70. J.P. Riddel, B.E. Aouizerat, C. Miaskowski, D.P. Lillicrap, J. Pediatr. Oncol. Nurs. 23, 123 (2007).

71. D. Brutin, B. Sobac, B. Loquet, J. Sampol, J. Fluid Mech. 667, 85 (2011).

72. J. Salvant Plisson, L. de Viguerie, L. Tahroucht, M. Menu, G. Ducouret, Colloids Surf. A: Physicochem. Eng. Asp. 458, 134 (2014).

73. L. Pauchard, C. Allain, C. R. Phys. 4, 231 (2003).

74. H. Valot, J. Roire, J. Petit, Encyclopedie De La Peinture, Vol. 1 (EREC, 1999).

75. A.L.R. Sibrant, L. Pauchard, EPL 116, 49002 (2016).

76. C. Brinker, G.W. Scherer, Sol-Gel Science: The Physics and Chemistry of Sol-Gel Processing (Academic Press, New York, 1990).

77. Johanna Salvant, Etienne Barthel, Michel Menu, Appl. Phys. A 104, 509 (2011).

78. Marguerite Leang, Frederique Giorgiutti-Dauphine, LayTheng Lee, Ludovic Pauchard, Soft Matter 13, 5802 (2017).

79. S. Bohn, L. Pauchard, Y. Couder, Phys. Rev. E 71, 046214 (2005).

80. Lucas Goehring, William J. Clegg, Alexander F. Routh, Phys. Rev. Lett. 110, 024301 (2013).

81. J.L. Beuth Jr., Int. J. Solids Struct. 29, 1657 (1992).

82. Lucas Goehring, Philos. Trans. R. Soc. A 371, 20120353 (2013).

83. Lucas Goehring, Rebecca Conroy, Asad Akhter, William J. Clegg, Alexander F. Routh, Soft Matter 6, 3562 (2010).

84. Gerhard Müller, J. Geophys. Res. 103, 15239 (1998).

85. A. Toramaru, T. Matsumoto, J. Geophys. Res. 109, B02205 (2004).

86. Lucas Goehring, Stephen W. Morris, Europhys. Lett. 69, 739 (2005).

87. Lucas Goehring, L. Mahadevan, Stephen W. Morris, Proc. Natl. Acad. Sci. U.S.A. 106, 387 (2009).
88. Maurice A. Biot, J. Appl. Phys. 27, 240 (1956).

89. A. Norris, J. Appl. Phys. 71, 1138 (1992).

90. Herbert F. Wang, Theory of Linear Poroelasticity (Princeton University Press, Oxford, 2000).

91. Lucas Goehring, Akio Nakahara, Tapati Dutta, So Kitsunezaki, Sujata Tarafdar, Desiccation cracks and their patterns: Formation and Modelling in Science and $\mathrm{Na}$ ture (Wiley-VCH, Singapore, 2015).

92. Maurice A. Biot, J. Appl. Phys. 12, 155 (1941).

93. Stephen S.L. Peppin, J.A. Elliott, M. Grae Worster, Phys. Fluids 17, 053301 (2005).

94. K. Terzaghi, The shearing resistance of saturated soils, in Proceedings of the International Conference on Soil Mechanics and Foundation Engineering, Vol. 1 (Graduate School of Engineering, Harvard University, Cambridge, MA, 1936) p. 54.

95. George W. Scherer, J. Non-Cryst. Solids 109, 171 (1989).

96. George W. Scherer, J. Am. Ceram. Soc. 73, 3 (1990).

97. George W. Scherer, J. Non-Cryst. Solids 144, 210 (1992).

98. James R. Rice, Michael P. Cleary, Rev. Geophys. Space Sci. 14, 227 (1976).

99. Alexander F. Routh, Rep. Prog. Phys. 76, 046603 (2013).

100. D. Brutin (Editor), Droplet Wetting and Evaporation: From Pure to Complex Fluids (Academic Press, 2015).

101. Lucas Goehring, Phys. Today 67, 39 (2014) issue No. 11.

102. L. Pauchard, M. Adda-Bedia, C. Allain, Y. Couder, Phys. Rev. E 67, 027103 (2003).

103. M.C. Milinkovitch, L. Manukyan, A. Debry, N. Di-Poï, S. Martin, D. Singh, D. Lambert, M. Zwicker, Science 339, 78 (2013)

104. Richard Bulkeley, Philos. Trans. R. Soc. Lond. 17, 708 (1693).

105. Sam Foley, T. Molyneux, Philos. Trans. R. Soc. Lond. 18, 170 (1694).

106. Thomas Molyneux, Philos. Trans. R. Soc. Lond. 20, 209 (1698).

107. Robert Mallet, Philos. Mag. 50, 122 (1875); 201 (1875).

108. T.H. Huxley, Physiography: An Introduction to the Study of Nature (MacMillan and Co., London, 1881).

109. J.W. French, Trans. Geol. Soc. Glasgow 17, 50 (1925).

110. S.I. Tomkeieff, Bull. Volcanol. 6, 89 (1940).

111. Gerhard Müller, J. Volcanol. Geotherm. Res. 86, 93 (1998).

112. Gerhard Müller, J. Struct. Geol. 23, 45 (2001).

113. Yuri Akiba, Jun Magome, Hiroshi Kobayashi, Hiroyuki Shima, Phys. Rev. E 96, 023003 (2017).

114. Tsuyoshi Mizuguchi, Akihiro Nishimoto, So Kitsunezaki, Yoshihiro Yamazaki, Ichio Aoki, Phys. Rev. E 71, 056122 (2005).

115. Lucas Goehring, Zhenquan Lin, Stephen W. Morris, Phys. Rev. E 74, 036115 (2006)

116. Lucas Goehring, Phys. Rev. E 80, 036116 (2009).

117. H.-A. Bahr, M. Hofmann, H.J. Weiss, U. Bahr, G. Fischer, H. Balke, Phys. Rev. E 79, 056103 (2009).

118. H.-A. Crostack, J. Nellesen, G. Fisher, M. Hofmann, H.G. Rademacher, W. Tillmann, Exp. Mech. 52, 917 (2012).

119. Martin Hofmann, Robert Anderssohn, Hans-Achim Bahr, Hans-Jürgen Weiß, Jens Nellesen, Phys. Rev. Lett. 115, 154301 (2015).

120. Dallas L. Peck, Takeshi Minakami, Geol. Soc. Am. Bull. 79, 1151 (1968).

121. Harry C. Hardee, J. Volcanol. Geotherm. Res. 7, 211 (1980). 
122. James M. DeGraff, Atilla Aydin, J. Geophys. Res. 98, 6411 (1993).

123. Paul Budkewitsch, Pierre-Yves Robin, J. Volcanol. Geotherm. Res. 59, 219 (1994).

124. L. Goehring, S.W. Morris, J. Geophys. Res. 113, B10203 (2008).

125. Philip E. Long, Bernard J. Wood, Geol. Soc. Am. Bull. 97, 1144 (1986).

126. Michael P. Ryan, Charles G. Sammis, Geol. Soc. Am. Bull. 89, 1295 (1978).

127. S. Bohn, J. Platkiewicz, B. Andreotti, M. Adda-Bedia, Y. Couder, Phys. Rev. E 71, 046215 (2005).

128. Akihiho Nishimoto, Tsuyoshi Mizuguchi, So Kitsunezaki, Phys. Rev. E 76, 016102 (2007).

129. J.W. Hutchinson, Z. Suo, Adv. Appl. Mech. 29, 63 (1992).

130. T. Bai, D.D. Pollard, H. Gao, Nature 403, 753 (2000).

131. Taixu Bai, David D. Pollard, J. Struct. Geol. 22, 43 (2000).

132. Martin Hofmann, Hans-Achim Bahr, Hans-Jürgen Weiss, Ute Bahr, Herbert Balke, Phys. Rev. E 83, 036104 (2011)

133. Denis Weaire, Stefan Hutzler, The Physics of Foams (Oxford University Press, 1999).

134. Atilla Aydin, James M. DeGraff, Science 239, 471 (1988).

135. J.P. O'Reilly, Trans. R. Irish Acad. 26, 641 (1879).

136. E.A. Jagla, Phys. Rev. Lett. 69, 056212 (2004).

137. E.M. Jones, Apollo 15 Lunar Surface Journal (Hadley Rille, 1996) http://www.hq.nasa.gov/office/pao/ History/alsj/a15/a15.rille.html.

138. M.P. Milazzo, L.P. Keszthelyi, W.L. Jaeger, M. Rosiek, S. Mattson, C. Verba, R.A. Beyer, P.E. Geissler, A.S. McEwen, Geology 37, 171 (2009).

139. Fredric M. Menger, Hong Zhang, Kevin L. Caran, Victor A. Seredyuk, Robert P. Apkarian, J. Am. Chem. Soc. 124, 1140 (2002).

140. K.V. Seshadri, J. Geol. Soc. India 49, 452 (1997).

141. Amalie Christensen, Christophe Raufaste, Marek Misztal, Franck Celestini, Maria Guidi, Clive Ellegaard, Joachim Mathiesen, J. Geophys. Res.: Solid Earth 121, 1462 (2016).

142. A. Yuse, M. Sano, Nature 362, 329 (1993).

143. A. Yuse, M. Sano, Physica D 108, 365 (1997).

144. Kelly A. Shorlin, John R. de Bruyn, Malcolm Graham, Stephen W. Morris, Phys. Rev. E 61, 6950 (2000).

145. D. Mal, S. Sinha, T. Dutta, S. Mitra, S. Tarafdar, J. Phys. Soc. Jpn. 76, 014801 (2007)

146. D. Mal, S. Sinha, S. Mitra, S. Tarafdar, Physica A 346, 110 (2005)

147. R.S. Sletten, B. Hallet, R.C. Fletcher, J. Geophys. Res. 108, 8044 (2003)

148. A.H. Lachenbruch, Mechanics of thermal contraction cracks and ice-wedge polygons in permafrost, GSA Special Papers, Vol. 70 (The Geological Society of America, 1962).

149. J. Ross Mackay, Can. J. Earth Sci. 11, 1366 (1974).

150. Thomas E. Berg, Robert F. Black, Preliminary measurements of growth of nonsorted polygons, Victoria Land, Antarctica, in Antarctic Soils and Soil Forming Processes, edited by J.C.F. Tedrow (American Geophysical Union, 1966) pp. 61-108.

151. J. Ross Mackay, C.R. Burn, Can. J. Earth Sci. 39, 95 (2002).

152. Yang Lu, Sihong Liu, Liping Weng, Liujiang Wang, Zhuo Li, Lei Xu, Eng. Geol. 208, 93 (2016).

153. Gregory V. Chavdarian, Dawn Y. Sumner, Sedimentology 58, 407 (2010).
154. Ankush Kumar, Rajashekhar Pujar, Nikita Gupta, Sujata Tarafdar, Giridhar U. Kulkarni, Appl. Phys. Lett. 111, 013502 (2017).

155. Koo Hyun Nam, Il H. Park, Seung Hwan Ko, Nature 485, 221 (2012).

156. Byoung Choul Kim, Toshiki Matsuoka, Christopher Moraes, Jiexi Huang, M.D. Thouless, Shuichi Takayama, Sci. Rep. 3, 221 (2013).

157. Minseok Kim, Dong-Joo Kim, Dogyeong Ha, Taesung Kim, Nanoscale 8, 9461 (2016).

158. Andy Goldsworthy, Time (Harry N. Abrams, London, 2008).

159. Thomas R. Watters, Sean C. Solomon, Christian Klimczak, Andrew M. Freed, James W. Head, Carolyn M. Ernst, David M. Blair, Timothy A. Goudge, Paul K. Byrne, Geology 40, 1123 (2012).

160. Andrew M. Freed, David M. Blair, Thomas R. Watters, Christian Klimczak, Paul K. Byrne, Sean C. Solomon, Maria T. Zuber, H.J. Melosh, J. Geophys. Res.: Planets 117, E00L06 (2012).

161. Pawan Nandakishore, Lucas Goehring, Soft Matter 12, 2253 (2016).

162. Gerald Schubert, Donald L. Turcotte, Peter Olson, Mantle Convection in the Earth and Planets (Cambridge University Press, 2001).

163. Subrahmanyan Chandrasekhar, Hydrodynamic and Hydromagnetic Stability (New York, Dover, 1961).

164. Anne Davaille, Angela Limare, Laboratory studies of mantle convection, in Treatise of Geophysics, Vol. 7: Mantle Dynamics, second edition (Elsevier, 2015).

165. A. Davaille, S.E. Smrekar, S. Tomlinson, Nat. Geosci. 10, 349 (2017).

166. David Bercovici, Paul Tackley, Yanick Ricard, The generation of plate tectonics from mantle dynamics, in Treatise of Geophysics, Vol. 7: Mantle Dynamics, second edition (Elsevier, 2015).

167. Tohru Okuzono, Kin'ya Ozawa, Masao Doi, Phys. Rev. Lett. 97, 136103 (2006).

168. R.W. Style, S.S.L. Peppin, Proc. R. Soc. London, Ser. A 467, 174 (2011).

169. Arthur A. Evans, Elliott Cheung, Kendra D. Nyberg, Amy C. Rowat, Soft Matter 13, 1056 (2017).

170. N. Tsapis, E.R. Dufresne, S.S. Sinha, C.S. Riera, J.W. Hutchinson, L. Mahadevan, D.A. Weitz, Phys. Rev. Lett. 94, 018302 (2005).

171. Erika Di Giuseppe, Anne Davaille, Spontaneous episodic initiation of one-sided subduction: insights from viscoelasto-plastic colloidal dispersions, submitted to Geophys. Res. Lett.

172. David T. Sandwell, Gerald Schubert, Science 257, 766 (1992).

173. H. Schouten, K.D. Klitgord, J.A. Whitehead, Nature 317, 225 (1985).

174. Ken C. Macdonald, P.J. Fox, L.J. Perram, M.F. Eisen, R.M. Haymon, S.P. Miller, S.M. Carbotte, M.H. Cormier, A.N. Shor, Nature 335, 217 (1988).

175. Suzanne M. Carbotte, Deborah K. Smith, Mathilde Cannat, Emily M. Klein, T.J. Wright, A. Ayele, D.J. Ferguson, T. Kidane, C. Vye-Brown, Tectonic and Magmatic Segmentation of the Global Ocean Ridge System: A Synthesis of Observations (Geological Society of London, 2015).

176. W. Oldenburg Douglas, N. Brune James, J. Geophys. Res. 80, 2575 (1975). 
177. Jian Lin, E.M. Parmentier, J. Geophys. Res.: Solid Earth 95, 4909 (1990).

178. Jason Phipps Morgan, Y. John Ghen, Nature 364, 706 (1993).

179. W. Roger Buck, Luc L. Lavier, Alexei N.B. Poliakov, Nature 434, 719 (2005).

180. Richard F. Katz, Rolf Ragnarsson, Eberhard Bodenschatz, New J. Phys. 7, 37 (2005).
181. Garrett Ito, Mark D. Behn, Geochem. Geophys. Geosyst. 9, Q09O12 (2008).

182. Taras Gerya, Science 329, 1047 (2010).

183. Tatiana Tentler, Valerio Acocella, J. Geophys. Res. 115, B01401 (2010).

184. Aurore Sibrant, Eric Mittelstaedt, Anne Davaille, Ludovic Pauchard, Alban Aubertin, Lionel Auffray, Raphael Pidoux, Nat. Geosci. 11, 274 (2018). 